\title{
Generalized Jacquet Modules of Parabolically Induced Representations
}

\author{
by
}

\author{
Noriyuki ABE
}

\begin{abstract}
In this paper we study a generalization of the Jacquet module of a parabolically induced representation and construct a filtration on it. The successive quotients of the filtration are written by using the twisting functor.
\end{abstract}

2010 Mathematics Subject Classification: Primary 22E46, Secondary 11F70.

Keywords: Jacquet module, parabolically induced representation, Bruhat filtration, twisting functor, Fréchet representation, Whittaker vector.

\section{§1. Introduction}

The Jacquet module of a representation of a semisimple (or reductive) Lie group was introduced by Casselman [Cas80]. One of the motivations of considering the Jacquet module is to investigate homomorphisms to principal series representations. The space of homomorphisms to principal series representations is an important invariant of a representation.

One of the powerful tools to study the Jacquet module of a parabolically induced representation is the Bruhat filtration [CHM00]. This is a filtration on the Jacquet module defined by the Bruhat decomposition. Casselman-HechtMiličić [CHM00] used the Bruhat filtration to determine the dimension of the (moderate-growth) Whittaker model of a principal series representation (another proof of Kostant's result [Kos78, Theorem I, Theorem J]). In this paper, we study the Bruhat filtration and show that its successive quotients are described by the twisting functor defined by Arkhipov [Ark04]. The successive quotients become

Communicated by M. Kashiwara. Received April 10, 2010. Revised October 7, 2010, and June $17,2011$.

N. Abe: Graduate School of Mathematical Sciences, University of Tokyo,

8-1 Komaba, Meguro-ku, Tokyo 153-8914, Japan;

e-mail: abenori@ms.u-tokyo.ac.jp

(C) 2012 Research Institute for Mathematical Sciences, Kyoto University. All rights reserved. 
"twisted" inductions, which have the same character as that of an induced representation but a different module structure.

Moreover, we investigate its generalization, which is related to the Whittaker model. In [Cas80], Casselman suggested generalizing the notion of the Jacquet module. For this generalized Jacquet module, we can also define a Bruhat filtration and the successive quotients of the filtration are described in terms of the generalized twisting functor.

This result gives a strategy to determine all Whittaker models of a parabolically induced representation. To determine it, it suffices to study the successive quotients and extensions of the filtration. In a special case, we can carry out these steps.

Now we state our results precisely. Let $G$ be a connected semisimple linear Lie group, $G=K A_{0} N_{0}$ an Iwasawa decomposition and $P_{0}=M_{0} A_{0} N_{0}$ a minimal parabolic subgroup and its Langlands decomposition. As usual, the complexifications of the Lie algebras is denoted by the corresponding German letter (for example, $\mathfrak{g}=\operatorname{Lie}(G) \otimes_{\mathbb{R}} \mathbb{C}$ ). Fix a character $\eta$ of $N_{0}$ and denote its differential also by $\eta$. Then for a representation $V$ of $G$, the generalized Jacquet modules $J_{\eta}^{\prime}(V)$ and $J_{\eta}^{*}(V)$ are defined as follows. Let $V_{K \text {-finite }}$ be the space of $K$-finite vectors in $V$.

Definition 1.1. Let $V$ be a finite-length moderate growth Fréchet representation of $G$ (see Casselman [Cas89, p. 391]). We define $\mathfrak{g}$-modules $J_{\eta}^{\prime}(V)$ and $J_{\eta}^{*}(V)$ by

$$
\begin{aligned}
& J_{\eta}^{\prime}(V)=\left\{\begin{array}{l|l}
v \in V^{\prime} & \begin{array}{l}
\text { for some } k \text { and for all } X \in \mathfrak{n}_{0}, \\
(X-\eta(X))^{k} v=0
\end{array}
\end{array},\right. \\
& J_{\eta}^{*}(V)=\left\{\begin{array}{l|l}
v \in\left(V_{K \text {-finite }}\right)^{*} & \begin{array}{l}
\text { for some } k \text { and for all } X \in \mathfrak{n}_{0}, \\
(X-\eta(X))^{k} v=0
\end{array}
\end{array}\right\},
\end{aligned}
$$

where $V^{\prime}$ is the continuous dual space of $V$ and $\left(V_{K \text {-finite }}\right)^{*}$ is the full dual space $\operatorname{Hom}_{\mathbb{C}}\left(V_{K \text {-finite }}, \mathbb{C}\right)$. If $\eta$ is the trivial representation, $J_{\eta}^{\prime}$ (resp. $\left.J_{\eta}^{*}\right)$ is denoted by $J^{\prime}$ (resp. $J^{*}$ ). The module $J^{*}(V)$ is called the Jacquet module of $V$.

(We will use the notation $Y^{*}=\operatorname{Hom}_{\mathbb{C}}(Y, \mathbb{C})$ for any $\mathbb{C}$-vector space $Y$ throughout this paper.)

In this paper, we consider $J_{\eta}^{\prime}(V)$ and $J_{\eta}^{*}(V)$ when $V$ is a parabolically induced representation. Let $P$ be a parabolic subgroup containing $P_{0}$ and take a Langlands decomposition $P=M A N$ such that $A_{0} \supset A$. For $\lambda \in \mathfrak{a}^{*}$ and an irreducible representation $\sigma$ of $M$, we define $I(\sigma, \lambda)=\operatorname{Ind}_{P}^{G}\left(\sigma \otimes e^{\lambda+\rho}\right)$ where $\rho \in \mathfrak{a}^{*}$ is the half sum of positive roots. In this paper, we deal with $J_{\eta}^{\prime}(I(\sigma, \lambda))$ and $J_{\eta}^{*}(I(\sigma, \lambda))$.

First we discuss $J_{\eta}^{\prime}(I(\sigma, \lambda))$. By definition, $I(\sigma, \lambda)$ is realized as the space of $C^{\infty}$-sections of a certain vector bundle on $G / P$. Hence an element of its continuous dual space is regarded as a distribution on $G / P$. Using the Bruhat decomposition 
on $G / P$, we can get a filtration $\left\{I_{i}\right\}$ of $J_{\eta}^{\prime}(I(\sigma, \lambda))$, which is called the Bruhat filtration. The first aim of this paper is to understand the structure of $I_{i} / I_{i-1}$.

We give a precise definition of $I_{i}$. Let $W\left(\right.$ resp. $\left.W_{M}\right)$ be the little Weyl group of $G($ resp. $M)$. Then $N_{0}$-orbits on $G / P$ are parameterized by $W / W_{M}$. Let $W(M)$ be a subset of $W$ consisting of $w$ such that $w(\alpha)$ is positive for any positive restricted root $\alpha$ of $M$. Then $W(M) \stackrel{\sim}{\rightarrow} W / W_{M}$. Enumerate $W(M)=\left\{w_{1}, \ldots, w_{r}\right\}$ so that $\bigcup_{j \leq i} N_{0} w_{j} P / P$ is a closed subset of $G / P$. Now we define a submodule $I_{i} \subset J_{\eta}^{\prime}(I(\sigma, \lambda))$ by

$$
I_{i}=\left\{x \in J_{\eta}^{\prime}(I(\sigma, \lambda)) \mid \operatorname{supp} x \subset \bigcup_{j \leq i} N_{0} w_{j} P\right\} .
$$

To describe $I_{i} / I_{i-1}$, we need a functor $T_{w_{i}, \eta}$ which is a generalization of the twisting functor [Ark04]. The generalized twisting functor $T_{w, \eta}$ is defined as follows. Let $\overline{\mathfrak{n}_{0}}$ be the nilradical of the parabolic subalgebra opposite to $\mathfrak{p}_{0}$ and $\left\{e_{1}, \ldots, e_{l}\right\}$ a basis of $\operatorname{Ad}(w) \overline{\mathfrak{n}}_{0} \cap \mathfrak{n}_{0}$ such that each $e_{i}$ is a root vector with respect to $\mathfrak{h}$, where $\mathfrak{h}$ is a Cartan subalgebra of $\mathfrak{g}$ which contains $\mathfrak{a}_{0}$. Moreover, we choose $e_{i}$ such that $\bigoplus_{i \leq j-1} \mathbb{C} e_{i}$ is an ideal of $\bigoplus_{i \leq j} \mathbb{C} e_{i}$ for all $j$. Let $U(\mathfrak{g})$ be the universal enveloping algebra of $\mathfrak{g}$ and $U(\mathfrak{g})_{e_{i}-\eta\left(e_{i}\right)}$ the localization of $U(\mathfrak{g})$ with respect to the multiplicative set $\left\{\left(e_{i}-\eta\left(e_{i}\right)\right)^{n} \mid n \in \mathbb{Z}_{>0}\right\}$. Put

$$
S_{w, \eta}=\left(U(\mathfrak{g})_{e_{1}-\eta\left(e_{1}\right)} / U(\mathfrak{g})\right) \otimes_{U(\mathfrak{g})} \cdots \otimes_{U(\mathfrak{g})}\left(U(\mathfrak{g})_{e_{l}-\eta\left(e_{l}\right)} / U(\mathfrak{g})\right) .
$$

Then $S_{w, \eta}$ is a $U(\mathfrak{g})$-bimodule and its $U(\mathfrak{g})$-bimodule structure is independent of the choice of $\left\{e_{1}, \ldots, e_{l}\right\}$. The twisting functor $T_{w, \eta}$ is an end-functor of the category of $\mathfrak{g}$-modules, defined by $T_{w, \eta} V=S_{w, \eta} \otimes_{U(\mathfrak{g})}(w V)$ for a $\mathfrak{g}$-module $V$, where $w V$ is the representation twisted by $w$ (i.e., $X v=\operatorname{Ad}(w)^{-1}(X) \cdot v$ for $X \in \mathfrak{g}$ and $v \in w V$, where the dot means the original action). If $\eta$ is the trivial representation, then $T_{w, \eta}$ is equal to the twisting functor defined by Arkhipov [Ark04]. In this case, we denote it by $T_{w}$.

Now we give the theorem.

Theorem 1.2 (Theorems 4.7 and 6.1). The filtration $\left\{I_{i}\right\}$ has the following properties.

(1) If the character $\eta$ is not unitary, then $J_{\eta}^{\prime}\left(I_{i} / I_{i-1}\right)=0$ for each $i=1, \ldots, r$. Therefore, $J_{\eta}^{\prime}(I(\sigma, \lambda))=0$.

(2) Assume that $\eta$ is unitary. The module $I_{i} / I_{i-1}$ is nonzero if and only if $\eta$ is trivial on $w_{i} N w_{i}^{-1} \cap N_{0}$ and $J_{w_{i}^{-1} \eta}^{\prime}\left(\sigma \otimes e^{\lambda+\rho}\right) \neq 0$.

(3) If $I_{i} / I_{i-1} \neq 0$ then $I_{i} / I_{i-1} \simeq T_{w_{i}, \eta}\left(U(\mathfrak{g}) \otimes_{U(\mathfrak{p})} J_{w_{i}^{-1} \eta}^{\prime}\left(\sigma \otimes e^{\lambda+\rho}\right)\right)$ where $\mathfrak{n}$ acts on $J_{w_{i}^{-1} \eta}^{\prime}\left(\sigma \otimes e^{\lambda+\rho}\right)$ trivially. 
Here are some remarks on notation. As $w_{i} \in W(M)$, we have $\operatorname{Ad}\left(w_{i}\right)\left(\mathfrak{m} \cap \mathfrak{n}_{0}\right)$ $\subset \mathfrak{n}_{0}$. Hence we can define a character $w_{i}^{-1} \eta$ of $\mathfrak{m} \cap \mathfrak{n}_{0}$ by $\left(w_{i}^{-1} \eta\right)(X)=\eta\left(\operatorname{Ad}\left(w_{i}\right) X\right)$. Using this character, we can define an $\mathfrak{m} \oplus \mathfrak{a}$-module $J_{w_{i}^{-1} \eta}^{\prime}\left(\sigma \otimes e^{\lambda+\rho}\right)$.

Under the assumptions that $P$ is a minimal parabolic subgroup, and that $\sigma$ is the trivial representation, $I(\sigma, \lambda)$ has the unique Langlands quotient and $\eta$ is the trivial representation, this theorem is proved in [Abe08]. The proof we gave in [Abe08] was algebraic, while we give an analytic and geometric proof in this paper.

Next we consider $J_{\eta}^{*}(I(\sigma, \lambda))$. For a $U(\mathfrak{g})$-module $V$, put $\Gamma_{\eta}(V)=\{v \in V \mid$ for some $k$ and for all $\left.X \in \mathfrak{n}_{0},(X-\eta(X))^{k} v=0\right\}$ and $C(V)=\left(\left(V^{*}\right)_{\mathfrak{h} \text {-finite }}\right)^{*}$. We prove the following theorem.

Theorem 1.3 (Theorem 7.5). There exists a filtration $0=\widetilde{I_{0}} \subset \widetilde{I}_{1} \subset \cdots \subset \widetilde{I}_{r}=$ $J_{\eta}^{*}(I(\sigma, \lambda))$ such that $\widetilde{I}_{i} / \widetilde{I_{i-1}} \simeq \Gamma_{\eta}\left(C\left(T_{w_{i}}\left(U(\mathfrak{g}) \otimes_{U(\mathfrak{p})} J^{*}\left(\sigma \otimes e^{\lambda+\rho}\right)\right)\right)\right)$ where $\mathfrak{n}$ acts on $J^{*}\left(\sigma \otimes e^{\lambda+\rho}\right)$ trivially.

Let us discuss an application. The space $\mathrm{Wh}_{\eta}(D)$ of Whittaker vectors for a $U(\mathfrak{g})$-module $D$ is defined by $\mathrm{Wh}_{\eta}(D)=\{x \in D \mid(X-\eta(X)) x=0$ for all $\left.X \in \mathfrak{n}_{0}\right\}$. If $V$ is a moderate growth Fréchet representation of $G$, an element of $\mathrm{Wh}_{\eta}\left(V^{\prime}\right)$ corresponds to a moderate growth homomorphism $V \rightarrow \operatorname{Ind}_{N_{0}}^{G} \eta$ and an element of $\mathrm{Wh}_{\eta}\left(\left(V_{K \text {-finite }}\right)^{*}\right)$ corresponds to an algebraic homomorphism $V_{K \text {-finite }} \rightarrow \operatorname{Ind}_{N_{0}}^{G} \eta$. In particular, when $\eta$ is the trivial representation, these correspond to homomorphisms to principal series representations. Obviously, we have $\mathrm{Wh}_{\eta}\left(V^{\prime}\right)=\mathrm{Wh}_{\eta}\left(J_{\eta}^{\prime}(V)\right)$ and $\mathrm{Wh}_{\eta}\left(\left(V_{K \text {-finite }}\right)^{*}\right)=\mathrm{Wh}_{\eta}\left(J_{\eta}^{*}(V)\right)$. Hence using the above theorems, we can determine the dimension of $\mathrm{Wh}_{\eta}\left(I(\sigma, \lambda)^{\prime}\right)$ and $\mathrm{Wh}_{\eta}\left(\left(I(\sigma, \lambda)_{K \text {-finite }}\right)^{*}\right)$ if $\lambda$ satisfies some (generic) condition.

Let us give such a formula. Let $\Sigma$ (resp. $\Sigma_{M}$ ) be the restricted root system for $\left(G, A_{0}\right)$ (resp. $\left.\left(M, M \cap A_{0}\right)\right), \Sigma^{+}$the positive system of $\Sigma$ corresponding to $N_{0}$, and $\Pi \subset \Sigma$ the set of simple roots determined by $\Sigma^{+}$. For $\alpha \in \Sigma$, the coroot of $\alpha$ is denoted by $\check{\alpha}$. Put $\Sigma_{M}^{+}=\Sigma_{M} \cap \Sigma^{+}$. Let $\widetilde{W}$ (resp. $\widetilde{W_{M}}$ ) be the (complex) Weyl group of $\mathfrak{g}$ (resp. $\mathfrak{m})$. Let $\tilde{\mu} \in(\mathfrak{m} \cap \mathfrak{h})^{*}$ be the infinitesimal character of $\sigma$. Using the decomposition $\mathfrak{h}=\mathfrak{a} \oplus(\mathfrak{m} \cap \mathfrak{h})$, we regard $(\mathfrak{m} \cap \mathfrak{h})^{*} \subset \mathfrak{h}^{*}$. Let $\Delta$ be the root system for $(\mathfrak{g}, \mathfrak{h})$. Put $\Sigma_{\eta}^{+}=\left(\sum_{\left.\eta\right|_{\mathfrak{g}} \neq 0, \beta \in \Pi} \mathbb{Z} \beta\right) \cap \Sigma^{+}$. Let $\rho_{0} \in \mathfrak{a}_{0}^{*}$ be the half sum of positive roots counted with multiplicities. Recall that $\nu \in\left(\mathfrak{m} \cap \mathfrak{a}_{0}\right)^{*}$ is called an exponent of $\sigma$ if $\nu+\left.\rho_{0}\right|_{\mathfrak{m} \cap \mathfrak{a}_{0}}$ is an $\left(\mathfrak{m} \cap \mathfrak{a}_{0}\right)$-weight of $\sigma /\left(\mathfrak{m} \cap \mathfrak{n}_{0}\right) \sigma$. Using $\mathfrak{a}_{0}=\left(\mathfrak{m} \cap \mathfrak{a}_{0}\right) \oplus \mathfrak{a}$, we regard $\nu$ as an element of $\mathfrak{a}_{0}^{*}$. We also have $\mathfrak{a}^{*} \subset \mathfrak{a}_{0}^{*}$.

Theorem 1.4 (Theorems 8.8 and 8.16). For $\lambda \in \mathfrak{a}^{*}$ and an irreducible representation $\sigma$ of $M$, the following formulas hold. 
(1) Assume that for any $w \in W$ such that $\left.\eta\right|_{w N w^{-1} \cap N_{0}}=1$, the following two conditions hold:

(a) $\langle\check{\alpha}, \lambda+\nu\rangle \notin \mathbb{Z}_{\leq 0}$ for each exponent $\nu$ of $\sigma$ and $\alpha \in \Sigma^{+} \backslash w^{-1}\left(\Sigma_{M}^{+} \cup \Sigma_{\eta}^{+}\right)$.

(b) $\lambda-\left.\left.\widetilde{w}(\lambda+\widetilde{\mu})\right|_{\mathfrak{a}} \notin \mathbb{Z}_{\leq 0}\left(\left(\Sigma^{+} \backslash \Sigma_{M}^{+}\right) \cap w^{-1} \Sigma^{+}\right)\right|_{\mathfrak{a}} \backslash\{0\}$ for all $\widetilde{w} \in \widetilde{W}$.

Then

$$
\operatorname{dim} \mathrm{Wh}_{\eta}\left(I(\sigma, \lambda)^{\prime}\right)=\sum_{w \in W(M),\left.\eta\right|_{w N w^{-1} \cap N_{0}}=1} \operatorname{dim} \mathrm{Wh}_{w^{-1} \eta}\left(\sigma^{\prime}\right) .
$$

(2) Assume that $(\lambda+\widetilde{\mu})-\widetilde{w}(\lambda+\widetilde{\mu}) \notin \mathbb{Z} \Delta$ for all $\widetilde{w} \in \widetilde{W} \backslash \widetilde{W_{M}}$. Then

$$
\operatorname{dim} \mathrm{Wh}_{\eta}\left(\left(I(\sigma, \lambda)_{K \text {-finite }}\right)^{*}\right)=\sum_{w \in W(M)} \operatorname{dim} \mathrm{Wh}_{w^{-1} \eta}\left(\left(\sigma_{M \cap K \text {-finite }}\right)^{*}\right) .
$$

For $\sigma$ finite-dimensional, we have the following theorem announced by T. Oshima (a talk at National University of Singapore, January 11, 2006). Let $\Delta_{M}$ be the root system for $(\mathfrak{m} \oplus \mathfrak{a}, \mathfrak{h})$ and take a positive system $\Delta_{M}^{+}$compatible with $\Sigma_{M}^{+}$. Put $\widetilde{\rho_{M}}=(1 / 2) \sum_{\alpha \in \Delta_{M}^{+}} \alpha$. For subsets $\Theta_{1}, \Theta_{2}$ of $\Pi$, put $\Sigma_{\Theta_{i}}=\mathbb{Z} \Theta_{i} \cap \Sigma$, $W\left(\Theta_{i}\right)=\left\{w \in W \mid w\left(\Theta_{i}\right) \subset \Sigma^{+}\right\}, W_{\Theta_{i}}$ the Weyl group of $\Sigma_{\Theta_{i}}$ and $W\left(\Theta_{1}, \Theta_{2}\right)=$ $\left\{w \in W\left(\Theta_{1}\right) \cap W\left(\Theta_{2}\right)^{-1} \mid w\left(\Sigma_{\Theta_{1}}\right) \cap \Sigma_{\Theta_{2}}=\emptyset\right\}$. The parabolic subgroup $P$ defines a subset of $\Pi$, denoted by $\Theta$. Let $w_{0} \in W$ be the longest element.

Theorem 1.5. Assume that $\sigma$ is an irreducible finite-dimensional representation of $M$ with highest weight $\widetilde{\nu}$. Let $\operatorname{dim}_{M_{0}}(\lambda+\widetilde{\nu})$ be the dimension of a finitedimensional irreducible representation of $M_{0} A_{0}$ with highest weight $\lambda+\widetilde{\nu}$.

(1) Assume that for all $w \in W$ such that $\left.\eta\right|_{w N_{0} w^{-1} \cap N_{0}}=1$ the following two conditions hold:

(a) $\left\langle\check{\alpha}, \lambda+w_{0} \widetilde{\nu}\right\rangle \notin \mathbb{Z}_{\leq 0}$ for all $\alpha \in \Sigma^{+} \backslash w^{-1}\left(\Sigma_{M}^{+} \cup \Sigma_{\eta}^{+}\right)$.

(b) $\lambda-\left.\left.\widetilde{w}\left(\lambda+\widetilde{\nu}+\widetilde{\rho_{M}}\right)\right|_{\mathfrak{a}} \notin \mathbb{Z}_{\leq 0}\left(\left(\Sigma^{+} \backslash \Sigma_{M}^{+}\right) \cap w^{-1} \Sigma^{+}\right)\right|_{\mathfrak{a}} \backslash\{0\}$ for all $\widetilde{w} \in \widetilde{W}$.

Then

$$
\operatorname{dim} \operatorname{Wh}_{\eta}\left(I(\sigma, \lambda)^{\prime}\right)=\# W(\operatorname{supp} \eta, \Theta) \times \operatorname{dim}_{M_{0}}(\lambda+\widetilde{\nu}) .
$$

(2) Assume that $(\lambda+\widetilde{\nu})-\widetilde{w}(\lambda+\widetilde{\nu}) \notin \Delta$ for all $\widetilde{w} \in \widetilde{W} \backslash \widetilde{W_{M}}$. Then

$$
\operatorname{dim} \mathrm{Wh}_{\eta}\left(\left(I(\sigma, \lambda)_{K \text {-finite }}\right)^{*}\right)=\# W(\operatorname{supp} \eta, \Theta) \times \# W_{\text {supp } \eta} \times \operatorname{dim}_{M_{0}}(\lambda+\widetilde{\nu}) .
$$

We summarize the content of this paper. In $\S 2$, we introduce the Bruhat filtration. From $\S 2$ to $\S 6$ we study the module $J_{\eta}^{\prime}(I(\sigma, \lambda))$. In $\S 3$ we prove that successive quotients of the Bruhat filtration are zero under some conditions. The structure of the successive quotients is investigated in $\S 4$. We give the definition and properties of the generalized twisting functor in $\S 5$, and in $\S 6$ we reveal the 
relation between the twisting functor and the successive quotients. We complete the proof of Theorem 1.2 in that section. Theorem 1.3 is proved in $\S 7$. In $\S 8$, the dimension of the space of Whittaker vectors is determined, and Theorems 1.4 and 1.5 are proved.

\section{List of symbols}

$\begin{array}{llll}\operatorname{supp}_{G} \eta=\operatorname{supp} \eta & \S 2,425 & I(\sigma, \lambda) & \S 2,427 \\ \mathcal{L} & \S 2,427 & W(M) & \S 2,427 \\ r & \S 2,427 & I_{i} & \S 2,427 \\ U_{i} & \S 2,427 & O_{i} & \S 2,427 \\ \operatorname{Res}_{i} & \S 2,428 & \delta_{i} & \S 2,428 \\ \mathcal{P}\left(O_{i}\right) & \S 2,428 & \eta_{i} & \S 2,428 \\ D_{i}(X) & \S 2,430 & R_{i}^{\prime}(X) & \S 3,433 \\ R(X) & \S 3,434 & \delta_{i}\left(E, f, u^{\prime}\right) & \S 3,434 \\ L(X) & \S 3,435 & \mathrm{Wh}_{\eta}(V) & \S 3,438 \\ \Phi_{w, w^{\prime}} & \S 4,439 & H & \S 4,440 \\ P_{\eta}=M_{\eta} A_{\eta} N_{\eta} & \S 4,442 & \mathfrak{p}_{\eta}=\mathfrak{m}_{\eta} \oplus \mathfrak{a}_{\eta} \oplus \mathfrak{n}_{\eta} & \S 4,442 \\ \mathfrak{l}_{\eta} & \S 4,442 & \bar{N}_{\eta} & \S 4,442 \\ \overline{\mathfrak{n}}_{\eta} & \S 4,442 & \Sigma_{\eta}^{+}, \Sigma_{\eta}^{-} & \S 4,442 \\ D(X, \lambda) & \S 4,444 & \mathfrak{g}_{\alpha}^{\mathfrak{y}} & \S 5,446 \\ \mathfrak{u}_{0} & \S 5,446 & \overline{\mathfrak{u}}_{0} & \S 5,446 \\ \mathfrak{u}_{0, \widetilde{w}} & \S 5,446 & S_{e_{k}-\psi\left(e_{k}\right)} & \S 5,446 \\ T_{\widetilde{w}, \psi} & J_{i} & \S 6,448 \\ J(V) & \S 5,446 & \mathcal{O}_{P_{0}}^{\prime} & \S 7,450 \\ \mathcal{O}_{\overline{P_{0}}}^{\prime} & \S 7,450 & D^{\prime}(V) & \S 7,450 \\ C(V) & \S 7,450 & \Gamma_{\eta}(V) & \S 7,450 \\ \widetilde{I}_{i} & \S 7,450 & \gamma_{1}, \gamma_{2}, \gamma_{3}, \gamma_{4} & \S 8,453 \\ W(\Theta) & \S 7,452 & W\left(\Theta_{1}, \Theta_{2}\right) & \S 8,465 \\ W_{\Theta} & \S 8,465 & \mathcal{D}^{\prime}(U, \mathcal{L}) & \S \mathrm{A}, 467 \\ \mathcal{T}(M, \mathcal{L}) & \S 8,465 & & \end{array}$

\section{Notation}

Throughout this paper we use the following notation. As usual we denote the ring of integers, the set of non-negative integers, the set of positive integers, the real number field and the complex number field by $\mathbb{Z}, \mathbb{Z}_{\geq 0}, \mathbb{Z}_{>0}, \mathbb{R}$ and $\mathbb{C}$, respectively. Let $G$ be a connected semisimple linear Lie group and $\mathfrak{g}$ the complexification of its Lie algebra. Fix a Cartan involution $\theta$ of $G$ and denote its derivation by the same letter $\theta$. Let $\mathfrak{g}=\mathfrak{k} \oplus \mathfrak{s}$ be the decomposition of $\mathfrak{g}$ into the +1 and -1 eigenspaces for $\theta$. Set $K=\{g \in G \mid \theta(g)=g\}$. Let $P_{0}=M_{0} A_{0} N_{0}$ be a 
minimal parabolic subgroup and its Langlands decomposition such that $M_{0} \subset K$ and $\operatorname{Lie}\left(A_{0}\right) \subset \mathfrak{s}$. Denote the complexifications of the Lie algebras of $P_{0}, M_{0}, A_{0}, N_{0}$ by $\mathfrak{p}_{0}, \mathfrak{m}_{0}, \mathfrak{a}_{0}, \mathfrak{n}_{0}$, respectively. Take a parabolic subgroup $P$ which contains $P_{0}$ and denote its Langlands decomposition by $P=M A N$. Here we assume $A \subset A_{0}$. Let $\mathfrak{p}, \mathfrak{m}, \mathfrak{a}, \mathfrak{n}$ be the complexifications of the Lie algebras of $P, M, A, N$. Put $\overline{P_{0}}=\theta\left(P_{0}\right)$, $\overline{N_{0}}=\theta\left(N_{0}\right), \bar{P}=\theta(P), \bar{N}=\theta(N), \overline{\mathfrak{p}_{0}}=\theta\left(\mathfrak{p}_{0}\right), \overline{\mathfrak{n}_{0}}=\theta\left(\mathfrak{n}_{0}\right), \overline{\mathfrak{p}}=\theta(\mathfrak{p})$ and $\overline{\mathfrak{n}}=\theta(\mathfrak{n})$.

In general, we denote the dual space $\operatorname{Hom}_{\mathbb{C}}(V, \mathbb{C})$ of a $\mathbb{C}$-vector space $V$ by $V^{*}$. Let $\Sigma \subset \mathfrak{a}_{0}^{*}$ be the restricted root system for $\left(\mathfrak{g}, \mathfrak{a}_{0}\right)$ and $\mathfrak{g}_{\alpha}$ the root space for $\alpha \in \Sigma$. Then $\sum_{\alpha \in \Sigma} \mathbb{R} \alpha$ is a real form of $\mathfrak{a}_{0}^{*}$. We denote the real part of $\lambda \in \mathfrak{a}_{0}^{*}$ with respect to this real form by $\operatorname{Re} \lambda$ and the imaginary part by $\operatorname{Im} \lambda$. Let $\Sigma^{+}$be the positive system determined by $\mathfrak{n}_{0}$. Put $\rho_{0}=\sum_{\alpha \in \Sigma^{+}}\left(\operatorname{dim} \mathfrak{g}_{\alpha} / 2\right) \alpha$ and $\rho=\left.\rho_{0}\right|_{\mathfrak{a}}$. The positive system $\Sigma^{+}$determines the set $\Pi$ of simple roots. Fix a total order on $\sum_{\alpha \in \Sigma} \mathbb{R} \alpha$ such that the following conditions hold: (1) If $\alpha>\beta$ and $\gamma \in \sum_{\alpha \in \Sigma} \mathbb{R} \alpha$ then $\alpha+\gamma>\beta+\gamma$. (2) If $\alpha>0$ and $c$ is a positive real number then $c \alpha>0$. (3) For all $\alpha \in \Sigma^{+}$we have $\alpha>0$. Write $W$ for the little Weyl group for $\left(\mathfrak{g}, \mathfrak{a}_{0}\right), e$ for the unit element of $W$ and $w_{0}$ for the longest element of $W$. For $w \in W$, we fix a representative in $N_{K}(\mathfrak{a})$ and denote it also by $w$. For $\alpha \in \Sigma$, let $\check{\alpha}$ be its coroot.

Let $\mathfrak{t}_{0}$ be a Cartan subalgebra of $\mathfrak{m}_{0}$ and $T_{0}$ the corresponding Cartan subgroup of $M_{0}$. Then $\mathfrak{h}=\mathfrak{t}_{0} \oplus \mathfrak{a}_{0}$ is a Cartan subalgebra of $\mathfrak{g}$. Let $\Delta$ be the root system for $(\mathfrak{g}, \mathfrak{h})$ and take a positive system $\Delta^{+}$compatible with $\Sigma^{+}$, i.e., if $\alpha \in \Delta^{+}$is such that $\left.\alpha\right|_{\mathfrak{a}_{0}} \neq 0$ then $\left.\alpha\right|_{\mathfrak{a}_{0}} \in \Sigma^{+}$. Let $\mathfrak{g}_{\alpha}^{\mathfrak{h}}$ be the root space of $\alpha \in \Delta$ and $\widetilde{W}$ the Weyl group of $\Delta$. Put $\widetilde{\rho}=(1 / 2) \sum_{\alpha \in \Delta^{+}} \alpha$. By the decompositions $\left(\mathfrak{m} \cap \mathfrak{a}_{0}\right)^{*} \oplus \mathfrak{a}^{*}=\mathfrak{a}_{0}^{*}$ and $\mathfrak{t}_{0}^{*} \oplus \mathfrak{a}_{0}^{*}=\mathfrak{h}^{*}$, we always regard $\mathfrak{a}^{*} \subset \mathfrak{a}_{0}^{*} \subset \mathfrak{h}^{*}$.

We use the same notation for $M$, i.e., $\Sigma_{M}$ is the restricted root system of $M$, $\Sigma_{M}^{+}=\Sigma_{M} \cap \Sigma^{+}, W_{M}$ is the little Weyl group of $M, \Delta_{M}$ is the root system of $M$, $\Delta_{M}^{+}=\Delta_{M} \cap \Delta^{+}, \widetilde{W_{M}}$ is the Weyl group of $M$ and $w_{M, 0}$ is the longest element of $W_{M}$.

We can define an anti-isomorphism of $U(\mathfrak{g})$ by $X \mapsto-X$ for $X \in \mathfrak{g}$. We denote this anti-isomorphism by $u \mapsto \check{u}$.

For a $\mathfrak{g}$-module $V$ and $g \in G$, we define a $\mathfrak{g}$-module $g V$ as follows: The representation space is $V$ and the action of $X \in \mathfrak{g}$ is $X \cdot v=\left(\operatorname{Ad}(g)^{-1} X\right) v$ for $v \in g V$.

For $\xi=\left(\xi_{1}, \ldots, \xi_{l}\right) \in \mathbb{Z}^{l}$, put $|\xi|=\xi_{1}+\cdots+\xi_{l}$.

\section{§2. Parabolic induction and the Bruhat filtration}

Fix a character $\eta$ of $\mathfrak{n}_{0}$ and put $\operatorname{supp}_{G} \eta=\operatorname{supp} \eta=\left\{\alpha \in \Pi|\eta|_{\mathfrak{g}_{\alpha}} \neq 0\right\}$. The character $\eta$ is called non-degenerate if $\operatorname{supp} \eta=\Pi$. We denote the character of $N_{0}$ whose differential is $\eta$ by the same letter $\eta$. 
Definition 2.1. Let $V$ be a finite-length moderate growth Fréchet representation of $G$ (see Casselman [Cas89, p. 391]). We define $\mathfrak{g}$-modules $J_{\eta}^{\prime}(V)$ and $J_{\eta}^{*}(V)$ by

$$
\begin{aligned}
& J_{\eta}^{\prime}(V)=\left\{\begin{array}{l|l}
v \in V^{\prime} & \begin{array}{l}
\text { for some } k \text { and for all } X \in \mathfrak{n}_{0}, \\
(X-\eta(X))^{k} v=0
\end{array}
\end{array}\right\}, \\
& J_{\eta}^{*}(V)=\left\{\begin{array}{l|l}
v \in\left(V_{K \text {-finite }}\right)^{*} & \begin{array}{l}
\text { for some } k \text { and for all } X \in \mathfrak{n}_{0}, \\
(X-\eta(X))^{k} v=0
\end{array}
\end{array}\right\},
\end{aligned}
$$

where $V^{\prime}$ is the continuous dual space of $V$.

Put $J^{\prime}(V)=J_{0}^{\prime}(V)$ and $J^{*}(V)=J_{0}^{*}(V)$ where 0 is the trivial representation of $\mathfrak{n}_{0}$. The module $J^{*}(V)$ is the (dual of the) Jacquet module defined by Casselman [Cas80]. By the automatic continuity theorem [Wal83, Theorem 4.8], we have $J^{\prime}(V)=J^{*}(V)$. The correspondences $V \mapsto J_{\eta}^{\prime}(V)$ and $V \mapsto J_{\eta}^{*}(V)$ are functors from the category of $G$-modules to the category of $\mathfrak{g}$-modules.

Remark 2.2. The character $\eta: \mathfrak{n}_{0} \rightarrow \mathbb{C}$ gives a $\mathbb{C}$-algebra homomorphism $U\left(\mathfrak{n}_{0}\right)$ $\rightarrow \mathbb{C}$. We denote this homomorphism again by $\eta$ and let Ker $\eta$ be its kernel. Then the following conditions are equivalent:

(1) For some $k$ and for all $X \in \mathfrak{n}_{0},(X-\eta(X))^{k} v=0$.

(2) For all $X \in \mathfrak{n}_{0}$ there exists $k$ such that $(X-\eta(X))^{k} v=0$.

(3) For some $k,(\operatorname{Ker} \eta)^{k} v=0$.

In fact, this holds for any nilpotent Lie algebra. Obviously, (3) implies (1) and (1) implies (2). We prove that (2) implies (3) by induction on dim $\mathfrak{n}_{0}$. Replacing $V$ with $V \otimes(-\eta)$, we may assume $\eta=0$. Take a codimension 1 ideal $\mathfrak{c} \subset \mathfrak{n}_{0}$ and $X \in \mathfrak{n}_{0} \backslash \mathfrak{c}$. Then $\mathfrak{c}^{k} v=0$ for some $k$ by inductive hypothesis. Put $V^{\prime}=U\left(\mathfrak{n}_{0}\right) v$. Then $V^{\prime}=U(\mathfrak{c}) U(\mathbb{C} X) v$. By $(2), U(\mathbb{C} X) v$ is finite-dimensional. Since $\mathfrak{c}$ is an ideal, $\mathfrak{c}^{k} U(\mathbb{C} X) v \subset U\left(\mathfrak{n}_{0}\right) \mathfrak{c}^{k} v=0$. Hence $V^{\prime}$ is finite-dimensional. Since each finitedimensional irreducible representation of a nilpotent algebra is a character, $V^{\prime}$ is given by an extension of characters. By the assumption (2), each irreducible subquotient of $V^{\prime}$ is trivial. Hence $\mathfrak{n}_{0}^{k^{\prime}} v=0$ for some $k^{\prime}$.

In this paper, we study the module $J_{\eta}^{\prime}(V)$ for a parabolically induced representation $V$. An element of $\mathfrak{a}^{*}$ is identified with a character of $A$. We denote the character of $A$ corresponding to $\lambda+\rho$ by $e^{\lambda+\rho}$ where $\lambda \in \mathfrak{a}^{*}$. For an irreducible moderate growth Fréchet representation $\sigma$ of $M$ and $\lambda \in \mathfrak{a}^{*}$, put

$$
I(\sigma, \lambda)=C^{\infty}-\operatorname{Ind}_{P}^{G}\left(\sigma \otimes e^{\lambda+\rho}\right) .
$$

(For moderate growth Fréchet representations, see Casselman [Cas89].) The representation $I(\sigma, \lambda)$ has a natural structure of a moderate growth Fréchet repre- 
sentation. Denote its continuous dual space by $I(\sigma, \lambda)^{\prime}$. Let $\mathcal{L}$ be a vector bundle on $G / P$ attached to the representation $\sigma \otimes e^{\lambda+\rho}$. Then $I(\sigma, \lambda)$ is the space of $C^{\infty}$-sections of $\mathcal{L}$.

Remark 2.3. A $C^{\infty}$-section of $\mathcal{L}$ corresponds to a $\sigma$-valued $C^{\infty}$-function $f$ on $G$ such that $f($ gman $)=\sigma(m)^{-1} e^{-(\lambda+\rho)(\log a)} f(g)$ for $g \in G, m \in M, a \in A$, $n \in N$. In particular a $C^{\infty}$-function on $G / P$ corresponds to a right $P$-invariant $C^{\infty}$-function on $G$. We use this identification throughout this paper.

We use the notation of Appendix A. We can regard $J_{\eta}^{\prime}(I(\sigma, \lambda))$ as a subspace of $\mathcal{D}^{\prime}(G / P, \mathcal{L})$ as follows. Let $G / P=\bigcup_{\gamma} U_{\gamma}$ be an open covering such that $\mathcal{L}$ is trivial on $U_{\gamma}$. For each $\gamma, C_{c}^{\infty}\left(U_{\gamma}, \mathcal{L}\right)$ is identified with a subspace $\left\{\varphi \in C^{\infty}(G / P, \mathcal{L}) \mid\right.$ $\left.\left.\varphi\right|_{(G / P) \backslash U_{\gamma}}=0\right\}$ of $C^{\infty}(G / P, \mathcal{L})=I(\sigma, \lambda)$. Hence an element of $I(\sigma, \lambda)^{\prime}$ gives an element of $\left(C_{c}^{\infty}\left(U_{\gamma}, \mathcal{L}\right)\right)^{\prime}$. By the definition of $\mathcal{D}^{\prime}(G / P, \mathcal{L})$, the collection of these elements in $\left(C_{c}^{\infty}\left(U_{\gamma}, \mathcal{L}\right)\right)^{\prime}$ over $\gamma^{\prime}$ s patches together to give an element of $\mathcal{D}^{\prime}(G / P, \mathcal{L})$. Hence we get $I(\sigma, \lambda)^{\prime} \rightarrow \mathcal{D}^{\prime}(G / P, \mathcal{L})$. It is easy to see that this is an injective $\mathfrak{g}$-module homomorphism.

Set $W(M)=\left\{w \in W \mid w\left(\Sigma_{M}^{+}\right) \subset \Sigma^{+}\right\}$. Then it is known that the multiplication map $W(M) \times W_{M} \rightarrow W$ is bijective [Kos61, Proposition 5.13]. By the Bruhat decomposition, we have

$$
G / P=\bigsqcup_{w \in W(M)} N_{0} w P / P
$$

(Recall that we fix a representative of $w \in W$, see Notation.) Enumerate $W(M)=$ $\left\{w_{1}, \ldots, w_{r}\right\}$ so that $\bigcup_{j \leq i} N_{0} w_{j} P / P$ is a closed subset of $G / P$ for each $i$. (For example, choose $w_{i}$ such that $\operatorname{dim}\left(N_{0} w_{1} P / P\right) \leq \cdots \leq \operatorname{dim}\left(N_{0} w_{r} P / P\right)$.) Then we can define a submodule $I_{i}$ of $J_{\eta}^{\prime}(I(\sigma, \lambda))$ by

$$
I_{i}=\left\{x \in J_{\eta}^{\prime}(I(\sigma, \lambda)) \mid \operatorname{supp} x \subset \bigcup_{j \leq i} N_{0} w_{j} P / P\right\} .
$$

The filtration $\left\{I_{i}\right\}$ is called a Bruhat filtration [CHM00]. In the rest of this section, we study the modules $I_{i} / I_{i-1}$. Put $U_{i}=w_{i} \bar{N} P / P$ and $O_{i}=N_{0} w_{i} P / P$. By the lemma below, $U_{i}$ is an open subset of $G / P$ containing $O_{i}$, and $U_{i} \cap O_{j}=\emptyset$ if $j<i$.

Lemma 2.4. Let $w, w^{\prime} \in W$ and assume that $w \overline{N_{0}} P \cap N_{0} w^{\prime} P \neq \emptyset$. Then $w^{\prime} \geq w$ with respect to the Bruhat order.

Proof. Take $H \in \operatorname{Lie}\left(A_{0}\right)$ such that $\alpha(H)<0$ for all $\alpha \in \Sigma^{+}$and put $a_{t}=\exp (t H)$ for $t \in \mathbb{R}_{>0}$. Then $\lim _{t \rightarrow \infty} a_{t} \bar{n} a_{t}^{-1}=1$ for all $\bar{n} \in \overline{N_{0}}$. By assumption, there exists $\bar{n} \in \overline{N_{0}}$ such that $w \bar{n} P / P \in N_{0} w^{\prime} P / P \subset G / P$. Since $N_{0} w^{\prime} P / P \subset G / P$ is stable 
under the action of $A_{0}$, we have $\left(w a_{t} w^{-1}\right) w \bar{n} P / P \in N_{0} w^{\prime} P / P$. Since $a_{t} \in A_{0} \subset P$, we have $w a_{t} \bar{n} a_{t}^{-1} P / P \in N_{0} w^{\prime} P / P$. Hence $w P / P=\lim _{t \rightarrow \infty} w a_{t} \bar{n} a_{t}^{-1} P / P \in$ $\overline{N_{0} w^{\prime} P / P}$ where $\overline{N_{0} w^{\prime} P / P}$ is the closure of $N_{0} w^{\prime} P / P$ in $G / P$, proving the lemma.

Hence, the restriction map $\operatorname{Res}_{i}: I_{i} \rightarrow \mathcal{D}^{\prime}\left(U_{i}, \mathcal{L}\right)$ induces an injective map

$$
\operatorname{Res}_{i}: I_{i} / I_{i-1} \rightarrow \mathcal{D}^{\prime}\left(U_{i}, \mathcal{L}\right) .
$$

Moreover, $\operatorname{Im} \operatorname{Res}_{i} \subset \mathcal{T}_{O_{i}}\left(U_{i}, \mathcal{L}\right)$ where $\mathcal{T}_{O_{i}}\left(U_{i}, \mathcal{L}\right)$ is the space of tempered $\mathcal{L}$ distributions on $U_{i}$ with respect to $G / P$ whose supports are contained in $O_{i}$.

The map $n \mapsto n w_{i} P / P$ yields isomorphisms $w_{i} \bar{N} w_{i}^{-1} \simeq U_{i}$ and $w_{i} \bar{N} w_{i}^{-1} \cap N_{0}$ $\simeq O_{i}$. Since the exponential map exp: $\operatorname{Ad}\left(w_{i}\right) \overline{\mathfrak{n}} \rightarrow w_{i} \bar{N} w_{i}^{-1}$ is a diffeomorphism, the space $U_{i}$ is diffeomorphic to a Euclidean space and $O_{i}$ is a subspace of $U_{i}$. Moreover, since $w_{i} \bar{N} w_{i}^{-1} \simeq\left(w_{i} \bar{N} w_{i}^{-1} \cap \overline{N_{0}}\right)\left(w_{i} \bar{N} w_{i}^{-1} \cap N_{0}\right)$, a basis of $\operatorname{Ad}\left(w_{i}\right) \overline{\mathfrak{n}} \cap \overline{\mathfrak{n}_{0}}$ satisfies the conditions of Appendix A.2. Hence $\mathcal{T}_{O_{i}}\left(U_{i}, \mathcal{L}\right) \hookrightarrow U\left(\operatorname{Ad}\left(w_{i}\right) \overline{\mathfrak{n}} \cap \overline{\mathfrak{n}_{0}}\right) \mathcal{D}^{\prime}\left(O_{i}, \mathcal{L}\right)$ $\simeq U\left(\operatorname{Ad}\left(w_{i}\right) \overline{\mathfrak{n}} \cap \overline{\mathfrak{n}_{0}}\right) \otimes_{\mathbb{C}} \mathcal{D}^{\prime}\left(O_{i}, \mathcal{L}\right)$ by Proposition A.3.

Fix a Haar measure on $w_{i} \bar{N} w_{i}^{-1} \cap N_{0}$. We define $\delta_{i} \in \mathcal{D}^{\prime}\left(O_{i}, \mathcal{L}\right)$ by

$$
\left\langle\delta_{i}, \varphi\right\rangle=\int_{w_{i} \bar{N} w_{i}^{-1} \cap N_{0}} \varphi\left(n w_{i}\right) d n
$$

for $\varphi \in C_{c}^{\infty}\left(O_{i}, \mathcal{L}\right)$. Recall that $U_{i}$ has the structure of a vector space and $O_{i}$ is a subspace. Let $\mathcal{P}\left(O_{i}\right)$ be the ring of polynomials on $O_{i}$ (cf. [CG90] or Appendix A.3). Define a $C^{\infty}$-function $\eta_{i}$ on $O_{i}$ by $\eta_{i}\left(n w_{i} P / P\right)=\eta(n)$ for $n \in w_{i} \bar{N} w_{i}^{-1} \cap N_{0}$. For a $C^{\infty}$-function $f$ on $O_{i}$ and $u^{\prime} \in \sigma^{\prime}$, we define $f \otimes u^{\prime} \in C^{\infty}\left(O_{i}, \sigma^{\prime}\right)$ by $\left(f \otimes u^{\prime}\right)(x)=$ $f(x) u^{\prime}$. Since $w_{i} \in W(M), \operatorname{Ad}\left(w_{i}\right)\left(\mathfrak{m} \cap \mathfrak{n}_{0}\right) \subset \mathfrak{n}_{0}$. Hence we can define a character $w_{i}^{-1} \eta$ of $\mathfrak{m} \cap \mathfrak{n}_{0}$ by $\left(w_{i}^{-1} \eta\right)(X)=\eta\left(\operatorname{Ad}\left(w_{i}\right) X\right)$. Using this character, we can define the Jacquet module $J_{w_{i}^{-1} \eta}^{\prime}\left(\sigma \otimes e^{\lambda+\rho}\right)$ of the $M A$-representation $\sigma \otimes e^{\lambda+\rho}$. It is an $\mathfrak{m} \oplus \mathfrak{a}$-module. Put

$$
I_{i}^{\prime}=\left\{\begin{array}{l|l}
\left.\sum_{k=1}^{l} E_{k}\left(\left(f_{k} \eta_{i}^{-1}\right) \otimes u_{k}^{\prime}\right) \delta_{i}\right) & \begin{array}{l}
E_{k} \in U\left(\operatorname{Ad}\left(w_{i}\right) \overline{\mathfrak{n}} \cap \overline{\mathfrak{n}_{0}}\right), f_{k} \in \mathcal{P}\left(O_{i}\right), \\
u_{k}^{\prime} \in J_{w_{i}^{-1} \eta}^{\prime}\left(\sigma \otimes e^{\lambda+\rho}\right)
\end{array}
\end{array}\right\} .
$$

(Recall that $\operatorname{Ad}\left(w_{i}\right) \overline{\mathfrak{n}} \cap \overline{\mathfrak{n}_{0}}$ is a normal direction to $O_{i}$ in $U_{i}$.) The space $I_{i}^{\prime}$ is a $U(\mathfrak{g})$-submodule of $\mathcal{D}^{\prime}\left(U_{i}, \mathcal{L}\right)$. Our aim is to prove that if $I_{i} / I_{i-1} \neq 0$ then $\operatorname{Res}_{i}$ gives an isomorphism $I_{i} / I_{i-1} \simeq I_{i}^{\prime}$.

Remark 2.5. Since $w_{i} \in W(M)$, it follows that $\operatorname{Ad}\left(w_{i}\right)\left(\mathfrak{m} \cap \mathfrak{n}_{0}\right) \subset \mathfrak{n}_{0}$ and $\operatorname{Ad}\left(w_{i}\right)\left(\mathfrak{m} \cap \overline{\mathfrak{n}_{0}}\right) \subset \overline{\mathfrak{n}_{0}}$. Hence

$$
\operatorname{Ad}\left(w_{i}\right) \mathfrak{m} \cap \mathfrak{n}_{0}=\left(\operatorname{Ad}\left(w_{i}\right)\left(\mathfrak{m} \cap \mathfrak{n}_{0}\right) \oplus \operatorname{Ad}\left(w_{i}\right)\left(\mathfrak{m} \cap \overline{\mathfrak{n}_{0}}\right)\right) \cap \mathfrak{n}_{0}=\operatorname{Ad}\left(w_{i}\right)\left(\mathfrak{m} \cap \mathfrak{n}_{0}\right) .
$$


By the same argument,

$$
\operatorname{Ad}\left(w_{i}\right) \mathfrak{m} \cap \mathfrak{p}_{0}=\operatorname{Ad}\left(w_{i}\right)\left(\mathfrak{m} \cap \mathfrak{p}_{0}\right) .
$$

We use these formulas frequently.

Lemma 2.6. (1) $\operatorname{Ad}\left(w_{i}\right)\left(\overline{\mathfrak{n}} \oplus\left(\mathfrak{m} \cap \mathfrak{n}_{0}\right)\right)$ is a subalgebra.

(2) $\operatorname{Ad}\left(w_{i}\right)\left(\overline{\mathfrak{n}} \oplus\left(\mathfrak{m} \cap \mathfrak{n}_{0}\right)\right)=\left(\operatorname{Ad}\left(w_{i}\right) \overline{\mathfrak{n}} \cap \overline{\mathfrak{n}_{0}}\right) \oplus\left(\operatorname{Ad}\left(w_{i}\right)(\overline{\mathfrak{n}} \oplus \mathfrak{m}) \cap \mathfrak{n}_{0}\right)$ and both direct summands are subalgebras.

(3) $\operatorname{Ad}\left(w_{i}\right)(\mathfrak{m} \oplus \overline{\mathfrak{n}}) \cap \mathfrak{n}_{0}=\left(\operatorname{Ad}\left(w_{i}\right) \overline{\mathfrak{n}} \cap \mathfrak{n}_{0}\right) \oplus\left(\operatorname{Ad}\left(w_{i}\right) \mathfrak{m} \cap \mathfrak{n}_{0}\right)$ and both direct summands are subalgebras.

Proof. (1) This subspace is the nilpotent radical of a minimal parabolic subalgebra.

(2) We have

$\operatorname{Ad}\left(w_{i}\right)\left(\overline{\mathfrak{n}} \oplus\left(\mathfrak{m} \cap \mathfrak{n}_{0}\right)\right)=\left(\operatorname{Ad}\left(w_{i}\right)\left(\overline{\mathfrak{n}} \oplus\left(\mathfrak{m} \cap \mathfrak{n}_{0}\right)\right) \cap \overline{\mathfrak{n}_{0}}\right) \oplus\left(\operatorname{Ad}\left(w_{i}\right)\left(\overline{\mathfrak{n}} \oplus\left(\mathfrak{m} \cap \mathfrak{n}_{0}\right)\right) \cap \mathfrak{n}_{0}\right)$.

By Remark 2.5, $\operatorname{Ad}\left(w_{i}\right)\left(\overline{\mathfrak{n}} \oplus\left(\mathfrak{m} \cap \mathfrak{n}_{0}\right)\right) \cap \overline{\mathfrak{n}_{0}}=\left(\operatorname{Ad}\left(w_{i}\right) \overline{\mathfrak{n}} \cap \overline{\mathfrak{n}_{0}}\right) \oplus\left(\operatorname{Ad}\left(w_{i}\right)\left(\mathfrak{m} \cap \mathfrak{n}_{0}\right) \cap \overline{\mathfrak{n}_{0}}\right)=$ $\operatorname{Ad}\left(w_{i}\right) \overline{\mathfrak{n}} \cap \overline{\mathfrak{n}}{ }_{0}$. We also have $\operatorname{Ad}\left(w_{i}\right)(\mathfrak{m} \oplus \overline{\mathfrak{n}}) \cap \mathfrak{n}_{0}=\left(\operatorname{Ad}\left(w_{i}\right)\left(\overline{\mathfrak{n}} \oplus\left(\mathfrak{m} \cap \mathfrak{n}_{0}\right)\right) \cap \mathfrak{n}_{0}\right) \oplus$ $\left(\operatorname{Ad}\left(w_{i}\right)\left(\mathfrak{m} \cap \overline{\mathfrak{n}_{0}}\right) \cap \mathfrak{n}_{0}\right)=\operatorname{Ad}\left(w_{i}\right)\left(\overline{\mathfrak{n}} \oplus\left(\mathfrak{m} \cap \mathfrak{n}_{0}\right)\right) \cap \mathfrak{n}_{0}$ since $\operatorname{Ad}\left(w_{i}\right)\left(\mathfrak{m} \cap \overline{\mathfrak{n}_{0}}\right) \subset \overline{\mathfrak{n}_{0}}$.

(3) This is obvious.

Lemma 2.7. Let $E_{1}, \ldots, E_{n}$ be a basis of $\operatorname{Ad}\left(w_{i}\right) \overline{\mathfrak{n}} \cap \overline{\mathfrak{n}_{0}}$ such that each $E_{s}$ is a restricted root vector for some root $\left(\right.$ say $\left.\alpha_{s}\right)$ and $F \in \operatorname{Ad}\left(w_{i}\right)(\overline{\mathfrak{n}} \oplus \mathfrak{m}) \cap \mathfrak{n}_{0}$. For $\xi=\left(\xi_{1}, \ldots, \xi_{n}\right) \in \mathbb{Z}_{\geq 0}^{n}$, set $E^{\xi}=E_{1}^{\xi_{1}} \cdots E_{n}^{\xi_{n}}$. Then for all $c \in \mathbb{C}$ we have

$$
\begin{aligned}
{\left[(F-c)^{k}, E^{\xi}\right] \in\left(\sum_{\xi^{\prime} \in A(\xi)} \mathbb{C} E^{\xi^{\prime}}\right) U\left(\operatorname{Ad}\left(w_{i}\right)(\overline{\mathfrak{n}} \oplus \mathfrak{m}) \cap \mathfrak{n}_{0}\right) } & \\
& \subset U\left(\operatorname{Ad}\left(w_{i}\right)\left(\overline{\mathfrak{n}} \oplus\left(\mathfrak{m} \cap \mathfrak{n}_{0}\right)\right)\right)
\end{aligned}
$$

where $A(\xi)=\left\{\xi^{\prime} \in \mathbb{Z}_{\geq 0}^{n}|| \xi^{\prime}|<| \xi \mid\right.$, or $\left(\left|\xi^{\prime}\right|=|\xi|\right.$ and $\left.\left.\sum \xi_{i}^{\prime} \alpha_{i}>\sum \xi_{i} \alpha_{i}\right)\right\}$.

Proof. Notice that $\alpha_{s}$ is negative.

We may assume $k=1$. We argue by induction on $|\xi|$. We have

$$
\left[F-c, E^{\xi}\right]=\left[F, E^{\xi}\right]=\sum_{s=1}^{n} \sum_{l=0}^{\xi_{s}-1} E_{1}^{\xi_{1}} \cdots E_{s-1}^{\xi_{s-1}} E_{s}^{l}\left[F, E_{s}\right] E_{s}^{\xi_{s}-l-1} E_{s+1}^{\xi_{s+1}} \cdots E_{n}^{\xi_{n}} .
$$

Hence, it is sufficient to prove

$$
\begin{aligned}
E_{1}^{\xi_{1}} \cdots E_{s-1}^{\xi_{s-1}} E_{s}^{l}\left[F, E_{s}\right] E_{s}^{\xi_{s}-l-1} E_{s+1}^{\xi_{s+1}} \cdots E_{n}^{\xi_{n}} & \\
& \in\left(\sum_{\xi^{\prime} \in A(\xi)} \mathbb{C} E^{\xi^{\prime}}\right) U\left(\operatorname{Ad}\left(w_{i}\right)(\overline{\mathfrak{n}} \oplus \mathfrak{m}) \cap \mathfrak{n}_{0}\right) .
\end{aligned}
$$

We may assume that $F$ is a restricted root vector. If $\left[F, E_{s}\right] \in \operatorname{Ad}\left(w_{i}\right) \overline{\mathfrak{n}} \cap \overline{\mathfrak{n}_{0}}$ then 
the left hand side is in $U\left(\operatorname{Ad}\left(w_{i}\right) \overline{\mathfrak{n}} \cap \overline{\mathfrak{n}_{0}}\right)$ and its $\mathfrak{a}_{0}$-weight is greater than that of $E^{\xi}$. Hence it belongs to $\sum_{\xi^{\prime} \in A(\xi)} \mathbb{C} E^{\xi^{\prime}}$.

Assume that $\left[F, E_{s}\right] \in \operatorname{Ad}\left(w_{i}\right)(\overline{\mathfrak{n}} \oplus \mathfrak{m}) \cap \mathfrak{n}_{0}$. Define $\xi^{(1)}, \xi^{(2)} \in \mathbb{Z}^{n}$ by $\xi^{(1)}=$ $\left(\xi_{1}, \ldots, \xi_{s-1}, l, 0, \ldots, 0\right)$ and $\xi^{(2)}=\left(0, \ldots, 0, \xi_{s}-l-1, \xi_{s+1}, \ldots, \xi_{n}\right)$. Using inductive hypothesis, we have

$$
\begin{aligned}
E^{\xi^{(1)}}\left[\left[F, E_{s}\right], E^{\left.\xi^{(2)}\right]}\right. & \in E^{\xi^{(1)}}\left(\sum_{\xi^{\prime} \in A\left(\xi^{(2)}\right)} \mathbb{C} E^{\xi^{\prime}}\right) U\left(\operatorname{Ad}\left(w_{i}\right)(\overline{\mathfrak{n}} \oplus \mathfrak{m}) \cap \mathfrak{n}_{0}\right) \\
& \subset\left(\sum_{\xi^{\prime} \in A\left(\xi^{(1)}+\xi^{(2)}\right)} \mathbb{C} E^{\xi^{\prime}}\right) U\left(\operatorname{Ad}\left(w_{i}\right)(\overline{\mathfrak{n}} \oplus \mathfrak{m}) \cap \mathfrak{n}_{0}\right) \\
& \subset\left(\sum_{\xi^{\prime} \in A(\xi)} \mathbb{C} E^{\xi^{\prime}}\right) U\left(\operatorname{Ad}\left(w_{i}\right)(\overline{\mathfrak{n}} \oplus \mathfrak{m}) \cap \mathfrak{n}_{0}\right) .
\end{aligned}
$$

On the other hand,

$$
\begin{aligned}
E^{\xi^{(1)}} E^{\xi^{(2)}}\left[F, E_{s}\right] \in( & \left.\sum_{\left|\xi^{\prime}\right| \leq\left|\xi^{(1)}+\xi^{(2)}\right|} \mathbb{C} E^{\xi^{\prime}}\right)\left[F, E_{s}\right] \\
& \subset\left(\sum_{\left|\xi^{\prime}\right| \leq\left|\xi^{(1)}+\xi^{(2)}\right|} \mathbb{C} E^{\xi^{\prime}}\right)\left(\operatorname{Ad}\left(w_{i}\right)(\overline{\mathfrak{n}} \oplus \mathfrak{m}) \cap \mathfrak{n}_{0}\right) .
\end{aligned}
$$

Since $\left|\xi^{(1)}+\xi^{(2)}\right|=|\xi|-1<|\xi|$, we get the assertion.

Let $X$ be an element of the normalizer of $\operatorname{Ad}\left(w_{i}\right) \overline{\mathfrak{n}} \cap \mathfrak{n}_{0}$ in $\mathfrak{g}$. For $f \in C^{\infty}\left(O_{i}\right)$ we define $D_{i}(X) f \in C^{\infty}\left(O_{i}\right)$ by

$$
\left(D_{i}(X) f\right)\left(n w_{i}\right)=\left.\frac{d}{d t} f\left(\exp (-t X) n \exp (t X) w_{i}\right)\right|_{t=0}
$$

where $n \in w_{i} \bar{N} w_{i}^{-1} \cap N_{0}$.

Lemma 2.8. Fix $f \in C^{\infty}\left(O_{i}\right), u^{\prime} \in\left(\sigma \otimes e^{\lambda+\rho}\right)^{\prime}$ and $X \in \mathfrak{g}$.

(1) If $X \in \mathfrak{a}_{0}$, then $X$ normalizes $\operatorname{Ad}\left(w_{i}\right) \overline{\mathfrak{n}} \cap \mathfrak{n}_{0}$ and

$$
\begin{aligned}
X\left(\left(f \otimes u^{\prime}\right) \delta_{i}\right)= & \left(\left(D_{i}(X) f\right) \otimes u^{\prime}\right) \delta_{i}+\left(f \otimes\left(\left(\operatorname{Ad}\left(w_{i}\right)^{-1} X\right) u^{\prime}\right)\right) \delta_{i} \\
& +\left(w_{i} \rho_{0}-\rho_{0}\right)(X)\left(f \otimes u^{\prime}\right) \delta_{i} .
\end{aligned}
$$

(2) If $X \in \operatorname{Ad}\left(w_{i}\right)\left(\mathfrak{m} \cap \mathfrak{n}_{0}\right)$ or $X \in \mathfrak{m}_{0}$, then $X$ normalizes $\operatorname{Ad}\left(w_{i}\right) \overline{\mathfrak{n}} \cap \mathfrak{n}_{0}$ and

$$
X\left(\left(f \otimes u^{\prime}\right) \delta_{i}\right)=\left(\left(D_{i}(X) f\right) \otimes u^{\prime}\right) \delta_{i}+\left(f \otimes\left(\left(\operatorname{Ad}\left(w_{i}\right)^{-1} X\right) u^{\prime}\right)\right) \delta_{i} .
$$

Proof. First we prove that $X$ normalizes $\operatorname{Ad}\left(w_{i}\right) \overline{\mathfrak{n}} \cap \mathfrak{n}_{0}$. If $X \in \mathfrak{m}_{0}+\mathfrak{a}_{0}$, then $X$ normalizes each restricted root space. Hence, $X$ normalizes $\operatorname{Ad}\left(w_{i}\right) \overline{\mathfrak{n}} \cap \mathfrak{n}_{0}$. If $X \in \operatorname{Ad}\left(w_{i}\right)\left(\mathfrak{m} \cap \mathfrak{n}_{0}\right)$, then $X \in \mathfrak{n}_{0}$ by Remark 2.5. Hence, $X$ normalizes $\mathfrak{n}_{0}$. Since $\mathfrak{m}$ normalizes $\overline{\mathfrak{n}}, X$ normalizes $\operatorname{Ad}\left(w_{i}\right) \overline{\mathfrak{n}}$. 
Put $g_{t}=\exp (t X)$ for $t \in \mathbb{R}$. Set $D(t)=\left|\operatorname{det}\left(\left.\operatorname{Ad}\left(g_{t}\right)^{-1}\right|_{\operatorname{Ad}\left(w_{i}\right) \overline{\mathfrak{n}} \cap \mathfrak{n}_{0}}\right)\right|$. Take $\varphi \in C_{c}^{\infty}\left(U_{i}, \mathcal{L}\right)$ and regard $\varphi$ as a $\sigma$-valued $C^{\infty}$-function on $w_{i} \bar{N} P$ (Remark 2.3). In each case, $w_{i}^{-1} g_{t} w_{i} \in P$. Hence $\varphi\left(x w_{i}^{-1} g_{t} w_{i}\right)=\left(\sigma \otimes e^{\lambda+\rho}\right)\left(w_{i}^{-1} g_{t} w_{i}\right)^{-1} \varphi(x)$. Then

$$
\begin{aligned}
\langle X((f & \left.\left.\left.\otimes u^{\prime}\right) \delta_{i}\right), \varphi\right\rangle=\left\langle\left(f \otimes u^{\prime}\right) \delta_{i},-X \varphi\right\rangle \\
& =\left.\frac{d}{d t} \int_{w_{i} \bar{N} w_{i}^{-1} \cap N_{0}} u^{\prime}\left(\varphi\left(g_{t} n w_{i}\right)\right) f\left(n w_{i}\right) d n\right|_{t=0} \\
& =\left.\frac{d}{d t} \int_{w_{i} \bar{N} w_{i}^{-1} \cap N_{0}} u^{\prime}\left(\varphi\left(\left(g_{t} n g_{t}^{-1}\right) w_{i}\left(w_{i}^{-1} g_{t} w_{i}\right)\right)\right) f\left(n w_{i}\right) d n\right|_{t=0} \\
& =\left.\frac{d}{d t} \int_{w_{i} \bar{N} w_{i}^{-1} \cap N_{0}} u^{\prime}\left(\left(\sigma \otimes e^{\lambda+\rho}\right)\left(w_{i}^{-1} g_{t} w_{i}\right)^{-1} \varphi\left(\left(g_{t} n g_{t}^{-1}\right) w_{i}\right)\right) f\left(n w_{i}\right) d n\right|_{t=0} \\
& =\left.\frac{d}{d t} \int_{w_{i} \bar{N} w_{i}^{-1} \cap N_{0}} u^{\prime}\left(\left(\sigma \otimes e^{\lambda+\rho}\right)\left(w_{i}^{-1} g_{t} w_{i}\right)^{-1} \varphi\left(n w_{i}\right)\right) f\left(g_{t}^{-1} n g_{t} w_{i}\right) D(t) d n\right|_{t=0} \\
& =\left.\frac{d}{d t} \int_{w_{i} \bar{N} w_{i}^{-1} \cap N_{0}}\left(\left(w_{i}^{-1} g_{t} w_{i}\right) u^{\prime}\right)\left(\varphi\left(n w_{i}\right)\right) f\left(g_{t}^{-1} n g_{t} w_{i}\right) D(t) d n\right|_{t=0} .
\end{aligned}
$$

This implies

$$
\begin{aligned}
X\left(\left(f \otimes u^{\prime}\right) \delta_{i}\right)= & \left(\left(D_{i}(X) f\right) \otimes u^{\prime}\right) \delta_{i}+\left(f \otimes\left(\left(\operatorname{Ad}\left(w_{i}\right)^{-1} X\right) u^{\prime}\right)\right) \delta_{i} \\
& +\left.\frac{d}{d t}\left|\operatorname{det}\left(\left.\operatorname{Ad}\left(g_{t}\right)^{-1}\right|_{\operatorname{Ad}\left(w_{i}\right) \overline{\mathfrak{n}} \cap \mathfrak{n}_{0}}\right)\right|\right|_{t=0}\left(\left(f \otimes u^{\prime}\right) \delta_{i}\right) .
\end{aligned}
$$

(1) Assume that $X \in \mathfrak{a}_{0}$. Since $w_{i} \in W(M)$, we have $w_{i} \bar{N} w_{i}^{-1} \cap N_{0}=$ $w_{i} \bar{N}_{0} w_{i}^{-1} \cap N_{0}$. This implies that $\operatorname{det}\left(\operatorname{Ad}\left(g_{t}\right)^{-1} \mid \operatorname{Ad}\left(w_{i}\right) \overline{\mathfrak{n}} \cap \mathfrak{n}_{0}\right)=e^{t\left(w_{i} \rho_{0}-\rho_{0}\right)(X)}$.

(2) First assume that $X \in \mathfrak{m}_{0}$. Since $M_{0} \ni g \mapsto \operatorname{det}\left(\left.\operatorname{Ad}(g)^{-1}\right|_{\operatorname{Ad}\left(w_{i}\right)} \overline{\mathfrak{n}} \cap \mathfrak{n}_{0}\right)$ is a 1-dimensional representation, it is unitary since $M_{0}$ is compact. Hence we have $\left|\operatorname{det}\left(\operatorname{Ad}\left(g_{t}\right)^{-1} \mid \operatorname{Ad}\left(w_{i}\right) \overline{\mathfrak{n}} \cap \mathfrak{n}_{0}\right)\right|=1$. Next assume $X \in \operatorname{Ad}\left(w_{i}\right) \mathfrak{m} \cap \mathfrak{n}_{0}$. Then $\operatorname{ad}(X)$ is nilpotent. Hence, $\operatorname{Ad}\left(g_{t}\right)-1$ is nilpotent. This implies $\operatorname{det}\left(\left.\operatorname{Ad}\left(g_{t}\right)^{-1}\right|_{\operatorname{Ad}\left(w_{i}\right) \overline{\mathfrak{n}} \cap \mathfrak{n}_{0}}\right)$ $=1$.

Lemma 2.9. Let $x \in \mathcal{T}_{O_{i}}\left(U_{i}, \mathcal{L}\right)$. Assume that there exists a positive integer $k$ such that $(X-\eta(X))^{k} x=0$ for all $X \in \operatorname{Ad}\left(w_{i}\right) \overline{\mathfrak{p}} \cap \mathfrak{n}_{0}$. Then $x \in I_{i}^{\prime}$. In particular $\operatorname{Im} \operatorname{Res}_{i} \subset I_{i}^{\prime}$.

Proof. Let $E_{s}$ and $\alpha_{s}$ be as in Lemma 2.7. For $\xi=\left(\xi_{1}, \ldots, \xi_{n}\right) \in \mathbb{Z}_{\geq 0}^{n}$, set $E^{\xi}=$ $E_{1}^{\xi_{1}} \cdots E_{n}^{\xi_{n}}$. Since $x \in \mathcal{T}_{O_{i}}\left(U_{i}, \mathcal{L}\right) \hookrightarrow U\left(\operatorname{Ad}\left(w_{i}\right) \overline{\mathfrak{n}} \cap \overline{\mathfrak{n}_{0}}\right) \mathcal{D}^{\prime}\left(O_{i}, \mathcal{L}\right)$, there exist $x_{\xi} \in$ $\mathcal{D}^{\prime}\left(O_{i}, \mathcal{L}\right)$ such that $x=\sum_{\xi} E^{\xi} x_{\xi}$ (finite sum).

First we prove $x_{\xi} \in\left(\mathcal{P}\left(O_{i}\right) \eta_{i}^{-1} \otimes\left(\sigma \otimes e^{\lambda+\rho}\right)^{\prime}\right) \delta_{i}$ by backward induction on the lexicographic order of $\left(|\xi|,-\sum_{s} \xi_{s} \alpha_{s}\right)$. Fix a nonzero element $F \in \operatorname{Ad}\left(w_{i}\right) \overline{\mathfrak{n}} \cap \mathfrak{n}_{0}$. Then $(F-\eta(F))^{k} x=\sum_{\xi}\left[(F-\eta(F))^{k}, E^{\xi}\right]\left(x_{\xi}\right)+\sum_{\xi} E^{\xi}\left((F-\eta(F))^{k} x_{\xi}\right)$. Assume 
that $(F-\eta(F))^{k} x=0$. Define the set $A(\xi)$ as in Lemma 2.7. By that lemma,

$$
\begin{aligned}
\sum_{\xi} E^{\xi}\left((F-\eta(F))^{k} x_{\xi}\right) & =-\sum_{\xi}\left[(F-\eta(F))^{k}, E^{\xi}\right]\left(x_{\xi}\right) \\
& \in \sum_{\xi}\left(\sum_{\xi^{\prime} \in A(\xi)} \mathbb{C} E^{\xi^{\prime}}\right) U\left(\operatorname{Ad}\left(w_{i}\right)(\overline{\mathfrak{n}} \oplus \mathfrak{m}) \cap \mathfrak{n}_{0}\right)\left(x_{\xi}\right) .
\end{aligned}
$$

Put $B(\xi)=\left\{\xi^{\prime}|| \xi^{\prime}|>| \xi \mid\right.$, or $\left(\left|\xi^{\prime}\right|=|\xi|\right.$ and $\left.\left.\sum \xi_{s}^{\prime} \alpha_{s}<\sum \xi_{s} \alpha_{s}\right)\right\}$. Notice that $U\left(\operatorname{Ad}\left(w_{i}\right)(\overline{\mathfrak{n}} \oplus \mathfrak{m}) \cap \mathfrak{n}_{0}\right)\left(x_{\xi}\right) \subset \mathcal{D}^{\prime}\left(O_{i}, \mathcal{L}\right)$. Since we have $U\left(\operatorname{Ad}\left(w_{i}\right) \overline{\mathfrak{n}} \cap \overline{\mathfrak{n}_{0}}\right) \mathcal{D}^{\prime}\left(O_{i}, \mathcal{L}\right) \simeq$ $U\left(\operatorname{Ad}\left(w_{i}\right) \overline{\mathfrak{n}} \cap \overline{\mathfrak{n}_{0}}\right) \otimes \mathcal{D}^{\prime}\left(O_{i}, \mathcal{L}\right)$, it follows that

$$
(F-\eta(F))^{k} x_{\xi} \in \sum_{\xi^{\prime} \in B(\xi)} U\left(\operatorname{Ad}\left(w_{i}\right)(\overline{\mathfrak{n}} \oplus \mathfrak{m}) \cap \mathfrak{n}_{0}\right)\left(x_{\xi^{\prime}}\right) .
$$

By inductive hypothesis, $x_{\xi^{\prime}} \in\left(\mathcal{P}\left(O_{i}\right) \eta_{i}^{-1} \otimes\left(\sigma \otimes e^{\lambda+\rho}\right)^{\prime}\right) \delta_{i}$ for all $\xi^{\prime} \in B(\xi)$. Hence we have $(F-\eta(F))^{k} x_{\xi} \in\left(\mathcal{P}\left(O_{i}\right) \eta_{i}^{-1} \otimes\left(\sigma \otimes e^{\lambda+\rho}\right)^{\prime}\right) \delta_{i}$. Therefore $x_{\xi} \in$ $\left(\mathcal{P}\left(O_{i}\right) \eta_{i}^{-1} \otimes\left(\sigma \otimes e^{\lambda+\rho}\right)^{\prime}\right) \delta_{i}$ by Corollary A.5.

Hence, we can write $x=\sum_{\xi} E^{\xi} \sum_{l}\left(f_{\xi, l} \eta_{i}^{-1} \otimes u_{\xi, l}^{\prime}\right) \delta_{i}$ (finite sum), where $f_{\xi, l} \in$ $\mathcal{P}\left(O_{i}\right)$ and $u_{\xi, l}^{\prime} \in\left(\sigma \otimes e^{\lambda+\rho}\right)^{\prime}$. Moreover, we may assume that $f_{\xi, l}$ is an $\mathfrak{a}_{0}$-weight vector with respect to $D_{i}$ and $\left\{f_{\xi, l}\right\}_{l}$ is linearly independent for each $\xi$. We prove $u_{\xi, l}^{\prime} \in J_{w_{i}^{-1} \eta}^{\prime}\left(\sigma \otimes e^{\lambda+\rho}\right)$. Take $F \in \mathfrak{m} \cap \mathfrak{n}_{0}$. By Lemma 2.8,

$$
\begin{aligned}
& \left(\operatorname{Ad}\left(w_{i}\right) F-\eta\left(\operatorname{Ad}\left(w_{i}\right) F\right)\right)^{k} x \\
& =\sum_{\xi, l}\left[\left(\operatorname{Ad}\left(w_{i}\right) F-\eta\left(\operatorname{Ad}\left(w_{i}\right) F\right)\right)^{k}, E^{\xi}\right]\left(\left(f_{\xi, l} \eta_{i}^{-1} \otimes u_{\xi, l}^{\prime}\right) \delta_{i}\right) \\
& \quad+\sum_{\xi, l} E^{\xi} \sum_{p=1}^{k}\left(\begin{array}{c}
k \\
p
\end{array}\right)\left(\left(\left(D_{i}\left(\operatorname{Ad}\left(w_{i}\right) F\right)\right)^{p}\left(f_{\xi, l}\right) \eta_{i}^{-1}\right) \otimes\left(F-\eta\left(\operatorname{Ad}\left(w_{i}\right) F\right)\right)^{k-p}\left(u_{\xi, l}^{\prime}\right)\right) \delta_{i} \\
& \quad+\sum_{\xi, l} E^{\xi}\left(f_{\xi, l} \eta_{i}^{-1} \otimes\left(F-\eta\left(\operatorname{Ad}\left(w_{i}\right) F\right)\right)^{k} u_{\xi, l}^{\prime}\right) \delta_{i} .
\end{aligned}
$$

Now we prove $u_{\xi, l}^{\prime} \in J_{w_{i}^{-1} \eta}^{\prime}\left(\sigma \otimes e^{\lambda+\rho}\right)$ by backward induction on the lexicographic order of $\left(|\xi|,-\sum \xi_{s} \alpha_{s},-\right.$ wt $\left.f_{\xi, l}\right)$ where wt $f_{\xi, l}$ is the $\mathfrak{a}_{0}$-weight of $f_{\xi, l}$ with respect to $D_{i}$. Take $k$ such that $\left(\operatorname{Ad}\left(w_{i}\right) F-\eta\left(\operatorname{Ad}\left(w_{i}\right) F\right)\right)^{k} x=0$. Then

$$
\begin{aligned}
\sum_{\xi, l} & E^{\xi}\left(f_{\xi, l} \eta_{i}^{-1} \otimes\left(F-\eta\left(\operatorname{Ad}\left(w_{i}\right) F\right)\right)^{k} u_{\xi, l}^{\prime}\right) \delta_{i} \\
= & -\sum_{\xi, l}\left[\left(\operatorname{Ad}\left(w_{i}\right) F-\eta\left(\operatorname{Ad}\left(w_{i}\right) F\right)\right)^{k}, E^{\xi}\right]\left(\left(f_{\xi, l} \eta_{i}^{-1} \otimes u_{\xi, l}^{\prime}\right) \delta_{i}\right) \\
& -\sum_{\xi, l} E^{\xi} \sum_{p=1}^{k}\left(\begin{array}{c}
k \\
p
\end{array}\right)\left(\left(\left(D_{i}\left(\operatorname{Ad}\left(w_{i}\right) F\right)\right)^{p}\left(f_{\xi, l}\right) \eta_{i}^{-1}\right)\left(F-\eta\left(\operatorname{Ad}\left(w_{i}\right) F\right)\right)^{k-p}\left(u_{\xi, l}^{\prime}\right)\right) \delta_{i} .
\end{aligned}
$$


Consequently,

$$
\begin{aligned}
& \left(f_{\xi, l} \otimes\left(F-\eta\left(\operatorname{Ad}\left(w_{i}\right) F\right)\right)^{k}\left(u_{\xi, l}^{\prime}\right)\right) \delta_{i} \\
& \in \sum_{\xi^{\prime} \in B(\xi), l^{\prime}} U\left(\operatorname{Ad}\left(w_{i}\right)(\overline{\mathfrak{n}} \oplus \mathfrak{m}) \cap \mathfrak{n}_{0}\right)\left(\left(f_{\xi^{\prime}, l^{\prime}} \eta_{i}^{-1} \otimes u_{\xi^{\prime}, l^{\prime}}^{\prime}\right) \delta_{i}\right) \\
& \quad+\sum_{\operatorname{wt} f_{\xi^{\prime}, l^{\prime}}<\operatorname{wt} f_{\xi, l}} \sum_{p}\left(\left(\left(D_{i}\left(\operatorname{Ad}\left(w_{i}\right) F\right)\right)^{p} f_{\xi^{\prime}, l^{\prime}} \eta_{i}^{-1}\right) \otimes\left(U(\mathbb{C} F) u_{\xi^{\prime}, l^{\prime}}^{\prime}\right)\right) \delta_{i} .
\end{aligned}
$$

By inductive hypothesis, $\left(F-\eta\left(\operatorname{Ad}\left(w_{i}\right) F\right)\right)^{k} u_{\xi, l}^{\prime} \in J_{w_{i}^{-1} \eta}^{\prime}\left(\sigma \otimes e^{\lambda+\rho}\right)$. This implies that $u_{\xi, l}^{\prime} \in J_{w_{i}^{-1} \eta}^{\prime}\left(\sigma \otimes e^{\lambda+\rho}\right)$.

In fact, $\operatorname{Im} \operatorname{Res}_{i}=I_{i}^{\prime}$ if $\operatorname{Im} \operatorname{Res}_{i} \neq 0$. This is proved in Section 4.

\section{§3. Vanishing lemma}

In this section, we fix $i \in\{1, \ldots, r\}$ and a basis $\left\{e_{1}, \ldots, e_{l}\right\}$ of $\operatorname{Ad}\left(w_{i}\right) \overline{\mathfrak{n}} \cap \mathfrak{n}_{0}$. Here we assume that each $e_{s}$ is a restricted root vector and denote its root by $\alpha_{s}$. Moreover, we assume that $\bigoplus_{s \leq t-1} \mathbb{C} e_{s}$ is an ideal of $\bigoplus_{s \leq t} \mathbb{C} e_{s}$ for all $t=1, \ldots, l$.

By the decomposition (as groups)

$$
\begin{aligned}
N_{0} /\left[N_{0}, N_{0}\right] \simeq & \left(\left(w_{i} \bar{P} w_{i}^{-1} \cap N_{0}\right) /\left(w_{i} \bar{P} w_{i}^{-1} \cap\left[N_{0}, N_{0}\right]\right)\right) \\
& \times\left(\left(w_{i} N w_{i}^{-1} \cap N_{0}\right) /\left(w_{i} N w_{i}^{-1} \cap\left[N_{0}, N_{0}\right]\right)\right)
\end{aligned}
$$

where $[\cdot, \cdot]$ is the commutator group, we can define a character $\eta^{\prime}$ of $N_{0}$ by $\eta^{\prime}(n)=$ $\eta(n)$ for $n \in w_{i} \bar{P} w_{i}^{-1} \cap N_{0}$ and $\eta^{\prime}(n)=1$ for $n \in w_{i} N w_{i}^{-1} \cap N_{0}$. First, we prove the following lemma. This gives a necessary condition for $I_{i} / I_{i-1} \neq 0$.

Lemma 3.1. Let $X \in \mathfrak{n}_{0}$. Then for all $x \in I_{i}^{\prime}$ there exists a positive integer $k$ such that $\left(X-\eta^{\prime}(X)\right)^{k} x=0$.

For the proof, we need some notation and lemmas. For $X \in \operatorname{Ad}\left(w_{i}\right) \overline{\mathfrak{n}} \cap \mathfrak{n}_{0}$, we define a differential operator $R_{i}^{\prime}(X)$ on $O_{i}$ by

$$
\left(R_{i}^{\prime}(X) \varphi\right)\left(n w_{i} P / P\right)=\left.\frac{d}{d t} \varphi\left(n \exp (t X) w_{i} P / P\right)\right|_{t=0}
$$

where $n \in w_{i} \bar{N} w_{i}^{-1} \cap N_{0}$. (Recall that $w_{i} \bar{N} w_{i}^{-1} \cap N_{0} \simeq O_{i}$ via the map $n \mapsto$ $n w_{i} P / P$.)

For $X \in \mathfrak{g}$, we define a differential operator $R(X)$ on $G$ by

$$
(R(X) \varphi)(g)=\left.\frac{d}{d t} \varphi(g \exp (t X))\right|_{t=0}
$$


for a $C^{\infty}$-function $\varphi$ on $G$. We define $R_{i}^{\prime}(E)\left(E \in U\left(\operatorname{Ad}\left(w_{i}\right) \overline{\mathfrak{n}} \cap \mathfrak{n}_{0}\right)\right)$ and $R(E)$ $(E \in U(\mathfrak{g}))$ in the usual way. For $E \in U(\mathfrak{g}), f \in C^{\infty}\left(O_{i}\right)$ and $u^{\prime} \in\left(\sigma \otimes e^{\lambda+\rho}\right)^{\prime}$, we define $\delta_{i}\left(E, f, u^{\prime}\right) \in \mathcal{D}_{O_{i}}^{\prime}\left(U_{i}, \mathcal{L}\right)$ by

$$
\left\langle\delta_{i}\left(E, f, u^{\prime}\right), \varphi\right\rangle=\int_{w_{i} \bar{N} w_{i}^{-1} \cap N_{0}} f\left(n w_{i}\right) u^{\prime}\left(\left(R\left(\operatorname{Ad}\left(w_{i}\right)^{-1} E\right) \varphi\right)\left(n w_{i}\right)\right) d n
$$

where $\varphi \in C_{c}^{\infty}\left(U_{i}, \mathcal{L}\right)$ and we regard $\varphi$ as a function on $w_{i} \bar{N} P$ (Remark 2.3).

Lemma 3.2. We have the following properties:

(1) $\delta_{i}\left(X E, f, u^{\prime}\right)=\delta_{i}\left(E, R_{i}^{\prime}(-X)(f), u^{\prime}\right)$ for $X \in \operatorname{Ad}\left(w_{i}\right) \overline{\mathfrak{n}} \cap \mathfrak{n}_{0}$.

(2) $\delta_{i}\left(E X, f, u^{\prime}\right)=\delta_{i}\left(E, f, \operatorname{Ad}\left(w_{i}\right)^{-1} X u^{\prime}\right)$ for $X \in \operatorname{Ad}\left(w_{i}\right) \mathfrak{p}$.

(3) The map $C^{\infty}\left(O_{i}\right) \otimes_{U\left(\operatorname{Ad}\left(w_{i}\right) \overline{\mathfrak{n}} \cap \mathfrak{n}_{0}\right)} U(\mathfrak{g}) \otimes_{U\left(\operatorname{Ad}\left(w_{i}\right) \mathfrak{p}\right)} w_{i}\left(\sigma \otimes e^{\lambda+\rho}\right)^{\prime} \rightarrow \mathcal{D}_{O_{i}}^{\prime}\left(U_{i}, \mathcal{L}\right)$ defined by $f \otimes E \otimes u^{\prime} \mapsto \delta_{i}\left(E, f, u^{\prime}\right)$ is injective.

Proof. (1) and (2) are obvious. To prove (3), the same argument in the proof of Proposition A.3 can be applied.

Lemma 3.3. Let $E \in \mathfrak{g}, E^{\prime} \in U(\mathfrak{g}), f \in C^{\infty}\left(O_{i}\right)$ and $u^{\prime} \in\left(\sigma \otimes e^{\lambda+\rho}\right)^{\prime}$. Then

$$
E \delta_{i}\left(E^{\prime}, f, u^{\prime}\right)=\sum_{\left(k_{1}, \ldots, k_{l}\right) \in \mathbb{Z}_{\geq 0}^{l}} \delta_{i}\left(\left(\operatorname{ad}\left(e_{l}\right)^{k_{l}} \cdots \operatorname{ad}\left(e_{1}\right)^{k_{1}} E\right) E^{\prime}, f \prod_{s=1}^{l} \frac{\left(-x_{s}\right)^{k_{s}}}{k_{s} !}, u^{\prime}\right),
$$

where $x_{i}$ is a polynomial on $O_{i}$ given by $\exp \left(a_{1} e_{1}\right) \cdots \exp \left(a_{l} e_{l}\right) w_{i} P / P \mapsto a_{i}$. (Notice that the right hand side is a finite sum since $\operatorname{ad}\left(e_{i}\right)$ is nilpotent.)

Proof. We remark that $\left(a_{1}, \ldots, a_{l}\right) \mapsto \exp \left(a_{1} e_{1}\right) \cdots \exp \left(a_{l} e_{l}\right)$ yields a diffeomorphism $\mathbb{R}^{l} \simeq w_{i} \bar{N} w_{i}^{-1} \cap N_{0}$, and a Haar measure of $w_{i} \bar{N} w_{i}^{-1} \cap N_{0}$ corresponds to the Euclidean measure of $\mathbb{R}^{l}$. Take $\varphi \in C_{c}^{\infty}\left(w_{i} \bar{N} P, \sigma \otimes e^{\lambda+\rho}\right)$. Put $n(a)=$ $\exp \left(a_{1} e_{1}\right) \cdots \exp \left(a_{l} e_{l}\right)$ for $a=\left(a_{1}, \ldots, a_{l}\right)$. By the definition, the action of $E \in \mathfrak{g}$ and $R_{i}\left(E^{\prime}\right)\left(E^{\prime} \in \mathfrak{g}\right)$ commute with each other. For $E \in \mathfrak{g}$, we have

$$
\begin{aligned}
& \left\langle E \delta_{i}\left(E^{\prime}, f, u^{\prime}\right), \varphi\right\rangle \\
& \quad=\int_{\mathbb{R}^{l}} u^{\prime}\left(\left((-E) R\left(\operatorname{Ad}\left(w_{i}\right)^{-1} E^{\prime}\right) \varphi\right)\left(n(a) w_{i}\right)\right) f\left(n(a) w_{i}\right) d a \\
& \left.\quad=\frac{d}{d t} \int_{\mathbb{R}^{l}} u^{\prime}\left(R\left(\operatorname{Ad}\left(w_{i}\right)^{-1} E^{\prime}\right) \varphi\right)\left(\exp (t E) n(a) w_{i}\right)\right)\left.f\left(n(a) w_{i}\right) d a\right|_{t=0} \\
& \left.\quad=\frac{d}{d t} \int_{\mathbb{R}^{l}} u^{\prime}\left(R\left(\operatorname{Ad}\left(w_{i}\right)^{-1} E^{\prime}\right) \varphi\right)\left(n(a) \exp \left(t \operatorname{Ad}(n(a))^{-1} E\right) w_{i}\right)\right)\left.f\left(n(a) w_{i}\right) d a\right|_{t=0} .
\end{aligned}
$$


The formula

$$
\begin{aligned}
\operatorname{Ad}(n(a))^{-1} E & =e^{-\operatorname{ad}\left(a_{l} e_{l}\right)} \cdots e^{-\operatorname{ad}\left(a_{1} e_{1}\right)} E \\
& =\sum_{\left(k_{1}, \ldots, k_{l}\right) \in \mathbb{Z}_{\geq 0}^{l}} \frac{\left(-a_{1}\right)^{k_{1}}}{k_{1} !} \cdots \frac{\left(-a_{l}\right)^{k_{l}}}{k_{l} !} \operatorname{ad}\left(e_{l}\right)^{k_{l}} \cdots \operatorname{ad}\left(e_{1}\right)^{k_{1}} E
\end{aligned}
$$

gives the lemma.

For $\mathbf{k}=\left(k_{1}, \ldots, k_{l}\right)$, we denote the operator $\operatorname{ad}\left(e_{l}\right)^{k_{l}} \cdots \operatorname{ad}\left(e_{1}\right)^{k_{1}}$ on $\mathfrak{g}$ by $\operatorname{ad}(e)^{\mathbf{k}}$ and the polynomial $\left(\left(-x_{1}\right)^{k_{1}} / k_{1} !\right) \cdots\left(\left(-x_{l}\right)^{k_{l}} / k_{l} !\right) \in \mathcal{P}\left(O_{i}\right)$ by $f_{\mathbf{k}}$; here the polynomial $x_{i}$ is defined in Lemma 3.3.

Lemma 3.4. Let $\mathbf{k}=\left(k_{1}, \ldots, k_{l}\right) \in \mathbb{Z}_{>0}^{l}$ and $X \in \mathfrak{n}_{0}$. Assume that $\operatorname{ad}(e)^{\mathbf{k}} X \in$ $\operatorname{Ad}\left(w_{i}\right) \overline{\mathfrak{n}} \cap \mathfrak{n}_{0}$. Then $R_{i}^{\prime}\left(\operatorname{ad}(e)^{\mathbf{k}} X\right) f_{\mathbf{k}}=0$.

Proof. We may assume that $X$ is a restricted root vector and denote its restricted root by $\alpha$. We consider the $\mathfrak{a}_{0}$-weight with respect to $D_{i}$. The polynomial $f_{\mathbf{k}}$ is an $\mathfrak{a}_{0}$-weight vector of weight $-\sum_{s} k_{s} \alpha_{s}$. This implies that $R_{i}^{\prime}\left(\operatorname{ad}(e)^{\mathbf{k}} X\right) f_{\mathbf{k}}$ is an $\mathfrak{a}_{0}$-weight vector of weight $\alpha$. However, $\mathcal{P}\left(O_{i}\right)$ has a decomposition into the direct sum of $\mathfrak{a}_{0^{-}}$weight spaces and its weight belongs to $\left\{\sum_{\beta \in \Sigma^{+}} b_{\beta} \beta \mid b_{\beta} \in \mathbb{Z}_{\leq 0}\right\} \not \supset \alpha$. Hence, $R_{i}^{\prime}\left(\operatorname{ad}(e)^{\mathbf{k}} X\right) f_{\mathbf{k}}=0$.

For $f \in \mathcal{P}\left(O_{i}\right)$ and $X \in \mathfrak{n}_{0}$ we define $L(X)(f)$ by

$$
L(X)(f)\left(n w_{i}\right)=\left.\frac{d}{d t} f\left(\exp (-t X) n w_{i}\right)\right|_{t=0} .
$$

Recall that the $C^{\infty}$-function $\eta_{i}$ on $O_{i}$ is defined by $\eta_{i}\left(n w_{i} P / P\right)=\eta(n)$ for $n \in w_{i} \bar{N} w_{i}^{-1} \cap N_{0}$, and the character $\eta^{\prime}$ of $N_{0}$ is defined by $\eta^{\prime}(n)=\eta(n)$ for $n \in w_{i} \bar{P} w_{i}^{-1} \cap N_{0}$ and $\eta^{\prime}(n)=1$ for $n \in w_{i} N w_{i}^{-1} \cap N_{0}$.

Lemma 3.5. Let $X \in \mathfrak{n}_{0}$ be a restricted root vector. For $f \in \mathcal{P}\left(O_{i}\right)$ and $u^{\prime} \in$ $J_{w_{i}^{-1} \eta}^{\prime}\left(\sigma \otimes e^{\lambda+\rho}\right)$, we have

$$
\begin{aligned}
& \left(X-\eta^{\prime}(X)\right) \delta_{i}\left(1, f \eta_{i}^{-1}, u^{\prime}\right)=\delta_{i}\left(1, L(X)(f) \eta_{i}^{-1}, u^{\prime}\right) \\
& \quad+\sum_{\operatorname{ad}(e)^{\mathbf{k}} X \in \operatorname{Ad}\left(w_{i}\right) \mathfrak{n}_{0} \cap \mathfrak{n}_{0}} \delta_{i}\left(1, f f_{\mathbf{k}} \eta_{i}^{-1},\left(\operatorname{Ad}\left(w_{i}\right)^{-1}\left(\operatorname{ad}(e)^{\mathbf{k}} X\right)-\eta^{\prime}\left(\operatorname{ad}(e)^{\mathbf{k}} X\right)\right) u^{\prime}\right) .
\end{aligned}
$$

(Again the sum on the right hand side is finite.)

In particular, if $X \in \operatorname{Ad}\left(w_{i}\right) \overline{\mathfrak{n}} \cap \mathfrak{n}_{0}$, then

$$
\left(X-\eta^{\prime}(X)\right) \delta_{i}\left(1, f \eta_{i}^{-1}, u^{\prime}\right)=\delta_{i}\left(1, L(X)(f) \eta_{i}^{-1}, u^{\prime}\right) .
$$


Proof. We have

$$
X \delta_{i}\left(1, f \eta_{i}^{-1}, u^{\prime}\right)=\sum_{\mathbf{k} \in \mathbb{Z}_{\geq 0}^{l}} \delta_{i}\left(\operatorname{ad}(e)^{\mathbf{k}} X, f f_{\mathbf{k}} \eta_{i}^{-1}, u^{\prime}\right) .
$$

by Lemma 3.3. Since $\operatorname{ad}(e)^{\mathbf{k}} X$ belongs to $\mathfrak{n}_{0}$ and is a restricted root vector, we have either $\operatorname{ad}(e)^{\mathbf{k}} X \in \operatorname{Ad}\left(w_{i}\right) \overline{\mathfrak{n}}_{0} \cap \mathfrak{n}_{0}$ or $\operatorname{ad}(e)^{\mathbf{k}} X \in \operatorname{Ad}\left(w_{i}\right) \mathfrak{n}_{0} \cap \mathfrak{n}_{0}$. Recall that $\operatorname{Ad}\left(w_{i}\right) \overline{\mathfrak{n}_{0}} \cap \mathfrak{n}_{0}=\operatorname{Ad}\left(w_{i}\right) \overline{\mathfrak{n}} \cap \mathfrak{n}_{0}$ since $w_{i} \in W(M)$. Assume that $\operatorname{ad}(e)^{\mathbf{k}} X \in$ $\operatorname{Ad}\left(w_{i}\right) \overline{\mathfrak{n}} \cap \mathfrak{n}_{0}$. By the definition of $\eta_{i}$ and $\eta^{\prime}$, we have $R_{i}^{\prime}\left(-\operatorname{ad}(e)^{\mathbf{k}} X\right)\left(\eta_{i}^{-1}\right)=$ $\eta\left(\operatorname{ad}(e)^{\mathbf{k}} X\right) \eta_{i}^{-1}=\eta^{\prime}\left(\operatorname{ad}(e)^{\mathbf{k}} X\right) \eta_{i}^{-1}$. Hence, using Lemma 3.4,

$$
\begin{aligned}
\delta_{i}\left(\operatorname{ad}(e)^{\mathbf{k}} X, f f_{\mathbf{k}} \eta_{i}^{-1}, u^{\prime}\right)= & \delta_{i}\left(1, R_{i}^{\prime}\left(-\operatorname{ad}(e)^{\mathbf{k}} X\right)\left(f f_{\mathbf{k}} \eta_{i}^{-1}\right), u^{\prime}\right) \\
= & \delta_{i}\left(1, R_{i}^{\prime}\left(-\operatorname{ad}(e)^{\mathbf{k}} X\right)(f) f_{\mathbf{k}} \eta_{i}^{-1}, u^{\prime}\right) \\
& +\eta^{\prime}\left(\operatorname{ad}(e)^{\mathbf{k}} X\right) \delta_{i}\left(1, f f_{\mathbf{k}} \eta_{i}^{-1}, u^{\prime}\right) .
\end{aligned}
$$

Next assume that $\operatorname{ad}(e)^{\mathbf{k}} X \in \operatorname{Ad}\left(w_{i}\right) \mathfrak{n}_{0} \cap \mathfrak{n}_{0}$. For $h \in \mathcal{P}\left(O_{i}\right)$, define $\widetilde{h} \in \mathcal{P}\left(U_{i}\right)$ by $\widetilde{h}\left(n n_{0} w_{i} P\right)=h\left(n w_{i} P\right)$ for $n \in w_{i} \bar{N} w_{i}^{-1} \cap N_{0}$ and $n_{0} \in w_{i} \bar{N} w_{i}^{-1} \cap \overline{N_{0}}$. Then $\left(R_{i}^{\prime}(Y) h\right)^{\sim}=R\left(\operatorname{Ad}\left(w_{i}\right)^{-1} Y\right) \widetilde{h}$ for all $Y \in \operatorname{Ad}\left(w_{i}\right) \overline{\mathfrak{n}_{0}} \cap \mathfrak{n}_{0}$. Since $\widetilde{f}\left(p n w_{i}\right)=\widetilde{f}\left(p w_{i}\right)$ for $p \in w_{i} \bar{N} P w_{i}^{-1}$ and $n \in w_{i} N_{0} w_{i}^{-1} \cap N_{0}$, we have $R\left(\operatorname{Ad}\left(w_{i}\right)^{-1}\left(-\operatorname{ad}(e)^{\mathbf{k}} X\right)\right)(\widetilde{f})$ $=0$. By Lemma 3.2(2),

$$
\begin{aligned}
\delta_{i}\left(\operatorname{ad}(e)^{\mathbf{k}} X, f f_{\mathbf{k}} \eta_{i}^{-1}, u^{\prime}\right)= & \delta_{i}\left(1, f f_{\mathbf{k}} \eta_{i}^{-1}, \operatorname{Ad}\left(w_{i}\right)^{-1}\left(\operatorname{ad}(e)^{\mathbf{k}} X\right) u^{\prime}\right) \\
= & \delta_{i}\left(1,\left.R\left(\operatorname{Ad}\left(w_{i}\right)^{-1}\left(-\operatorname{ad}(e)^{\mathbf{k}} X\right)\right)(\widetilde{f})\right|_{O_{i}} f_{\mathbf{k}} \eta_{i}^{-1}, u^{\prime}\right) \\
& +\delta_{i}\left(1, f f_{\mathbf{k}} \eta_{i}^{-1}, \operatorname{Ad}\left(w_{i}\right)^{-1}\left(\operatorname{ad}(e)^{\mathbf{k}} X\right) u^{\prime}\right) .
\end{aligned}
$$

By the same calculation as in the proof of Lemma 3.3,

$$
L(X)(f)^{\sim}=L(X)(\widetilde{f})=\sum_{\mathbf{k} \in \mathbb{Z}_{\geq 0}^{l}} R\left(\operatorname{Ad}\left(w_{i}\right)^{-1}\left(-\operatorname{ad}(e)^{\mathbf{k}} X\right)\right)(\widetilde{f}) \widetilde{f_{\mathbf{k}}}
$$

Hence

$$
\begin{aligned}
\sum_{\mathbf{k} \in \mathbb{Z}_{\geq 0}^{l}} \delta_{i}\left(1,\left.R\left(\operatorname{Ad}\left(w_{i}\right)^{-1}\left(-\operatorname{ad}(e)^{\mathbf{k}} X\right)\right)(\tilde{f})\right|_{O_{i}} f_{\mathbf{k}} \eta_{i}^{-1}, u^{\prime}\right) \\
=\delta_{i}\left(1,\left.(L(X)(f))^{\sim}\right|_{O_{i}} \eta_{i}^{-1}, u^{\prime}\right)=\delta_{i}\left(1, L(X)(f) \eta_{i}^{-1}, u^{\prime}\right) .
\end{aligned}
$$

These imply that

$$
\begin{aligned}
& \left(X-\eta^{\prime}(X)\right) \delta_{i}\left(1, f \eta_{i}^{-1}, u^{\prime}\right)=\delta_{i}\left(1, L(X)(f) \eta_{i}^{-1}, u^{\prime}\right) \\
& +\sum_{\operatorname{ad}(e)^{\mathbf{k}} X \in \operatorname{Ad}\left(w_{i}\right) \mathfrak{n}_{0} \cap \mathfrak{n}_{0}} \delta_{i}\left(1, f f_{\mathbf{k}} \eta_{i}^{-1}, \operatorname{Ad}\left(w_{i}\right)^{-1}\left(\operatorname{ad}(e)^{\mathbf{k}} X\right) u^{\prime}\right) \\
& \quad+\sum_{\operatorname{ad}(e)^{\mathbf{k}} X \in \operatorname{Ad}\left(w_{i}\right) \overline{\mathfrak{n}} \cap \mathfrak{n}_{0}} \eta^{\prime}\left(\operatorname{ad}(e)^{\mathbf{k}} X\right) \delta_{i}\left(1, f f_{\mathbf{k}} \eta_{i}^{-1}, u^{\prime}\right)-\eta^{\prime}(X) \delta_{i}\left(1, f \eta_{i}^{-1}, u^{\prime}\right) .
\end{aligned}
$$


Since $\eta^{\prime}$ is a character, if $\mathbf{k} \neq(0, \ldots, 0)$ then $\eta^{\prime}\left(\operatorname{ad}(e)^{\mathbf{k}} X\right)=0$. Hence

$$
\sum_{\mathbf{k} \in \mathbb{Z}_{\geq 0}^{n}} \eta^{\prime}\left(\operatorname{ad}(e)^{\mathbf{k}} X\right) \delta_{i}\left(1, f f_{\mathbf{k}} \eta_{i}^{-1}, u^{\prime}\right)=\eta^{\prime}(X) \delta_{i}\left(1, f \eta_{i}^{-1}, u^{\prime}\right) .
$$

This implies

$$
\begin{array}{r}
\left(\sum_{\operatorname{ad}(e)^{\mathbf{k}} X \in \operatorname{Ad}\left(w_{i}\right) \overline{\mathfrak{n}} \cap \mathfrak{n}_{0}} \eta^{\prime}\left(\operatorname{ad}(e)^{\mathbf{k}} X\right) \delta_{i}\left(1, f f_{\mathbf{k}} \eta_{i}^{-1}, u^{\prime}\right)\right)-\eta^{\prime}(X) \delta_{i}\left(1, f \eta_{i}^{-1}, u^{\prime}\right) \\
=-\sum_{\operatorname{ad}(e)^{\mathbf{k}} X \in \operatorname{Ad}\left(w_{i}\right) \mathfrak{n}_{0} \cap \mathfrak{n}_{0}} \eta^{\prime}\left(\operatorname{ad}(e)^{\mathbf{k}} X\right) \delta_{i}\left(1, f f_{\mathbf{k}} \eta_{i}^{-1}, u^{\prime}\right),
\end{array}
$$

proving the lemma.

Proof of Lemma 3.1. Since $\operatorname{ad}\left(\mathfrak{n}_{0}\right)$ is nilpotent, the subspace

$$
\left\{x \in I_{i}^{\prime} \mid \text { for some } k \text { and for all } X \in \mathfrak{n}_{0},\left(X-\eta^{\prime}(X)\right)^{k} x=0\right\}
$$

is $\mathfrak{g}$-stable. Hence we may assume that $x=\left(\left(f \eta_{i}^{-1}\right) \otimes u^{\prime}\right) \delta_{i}=\delta_{i}\left(1, f \eta_{i}^{-1}, u^{\prime}\right)$ for some $f \in \mathcal{P}\left(O_{i}\right)$ and $u^{\prime} \in J_{w_{i}^{-1} \eta}^{\prime}\left(\sigma \otimes e^{\lambda+\rho}\right)$.

Now define $V=U\left(\operatorname{Ad}\left(w_{i}\right)^{-1} \mathfrak{n}_{0} \cap \mathfrak{n}_{0}\right) u^{\prime} \subset J_{w_{i}^{-1} \eta}^{\prime}\left(\sigma \otimes e^{\lambda+\rho}\right)$ where $\mathfrak{n}$ acts on $J_{w_{i}^{-1} \eta}^{\prime}\left(\sigma \otimes e^{\lambda+\rho}\right)$ trivially. Then $V$ is finite-dimensional. Since $\mathfrak{n}$ acts on $V$ trivially, $X-\left(w_{i}^{-1} \eta^{\prime}\right)(X)$ acts on $V$ as a nilpotent operator for $X \in \operatorname{Ad}\left(w_{i}\right)^{-1} \mathfrak{n}_{0} \cap \mathfrak{n}_{0}$ by the definition of $\eta^{\prime}$. By applying Engel's theorem for $V \otimes\left(-w_{i}^{-1} \eta^{\prime}\right)$, there exists a filtration $0=V_{0} \subset V_{1} \subset \cdots \subset V_{p}=V$ such that $\left(V_{s} / V_{s-1}\right) \otimes\left(-\left.w_{i}^{-1} \eta^{\prime}\right|_{\operatorname{Ad}\left(w_{i}\right)^{-1} \mathfrak{n}_{0} \cap \mathfrak{n}_{0}}\right)$ is the trivial representation of $\operatorname{Ad}\left(w_{i}\right)^{-1} \mathfrak{n}_{0} \cap \mathfrak{n}_{0}$. Then $V_{s} /\left.V_{s-1} \simeq w_{i}^{-1} \eta^{\prime}\right|_{\operatorname{Ad}\left(w_{i}\right)^{-1} \mathfrak{n}_{0} \cap \mathfrak{n}_{0}}$ for all $s=1, \ldots, p$. We prove the lemma by induction on $p=\operatorname{dim} V$.

We may assume that $X$ is a restricted root vector. By Lemma 3.5,

$$
\begin{aligned}
\left(X-\eta^{\prime}(X)\right) \delta_{i}\left(1, f \eta_{i}^{-1}, u^{\prime}\right) \in \delta_{i}\left(1, L(X)(f) \eta_{i}^{-1}, u^{\prime}\right) & \\
& +\sum_{h \in \mathcal{P}\left(O_{i}\right), v^{\prime} \in V_{p-1}} \mathbb{C} \delta_{i}\left(1, h \eta_{i}^{-1}, v^{\prime}\right) .
\end{aligned}
$$

Since $f$ is a polynomial, $(L(X))^{c}(f)=0$ for some positive integer $c$. Then we have $\left(X-\eta^{\prime}(X)\right)^{c} \delta_{i}\left(1, f \eta_{i}^{-1}, u^{\prime}\right) \in \sum_{h \in \mathcal{P}\left(O_{i}\right), v^{\prime} \in V_{p-1}} \mathbb{C} \delta_{i}\left(1, h \eta_{i}^{-1}, v^{\prime}\right)$. By inductive hypothesis, the lemma follows.

From the lemma, we get the following vanishing lemma. Recall that we define the character $w_{i}^{-1} \eta$ of $\mathfrak{m} \cap \mathfrak{n}_{0}$ by $\left(w_{i}^{-1} \eta\right)(X)=\eta\left(\operatorname{Ad}\left(w_{i}\right) X\right)$ and we have the injective homomorphism $\operatorname{Res}_{i}: I_{i} / I_{i-1} \rightarrow I_{i}^{\prime}$. 
Lemma 3.6. Assume that $I_{i} / I_{i-1} \neq 0$. Then:

(1) The character $\eta$ is unitary.

(2) The character $\eta$ is zero on $\operatorname{Ad}\left(w_{i}\right) \mathfrak{n} \cap \mathfrak{n}_{0}$. (This is equivalent to $\eta=\eta^{\prime}$.)

(3) The module $J_{w_{i}^{-1} \eta}^{\prime}\left(\sigma \otimes e^{\lambda+\rho}\right)$ is not zero.

Proof. (2) By Lemma 3.1 and the definition of $J_{\eta}^{\prime}$, if $I_{i} / I_{i-1} \neq 0$ then $\eta=\eta^{\prime}$.

(3) This is clear from Lemma 2.9.

(1) It is sufficient to prove that if $\eta$ is not unitary then $J_{\eta}^{\prime}(V)=0$ for all irreducible representations $V$ of $G$. By Casselman's subrepresentation theorem, $V$ is a subrepresentation of a principal series representation. Since $J_{\eta}^{\prime}$ is an exact functor, we may assume $V$ is a principal series representation $\operatorname{Ind}_{P_{0}}^{G}\left(\sigma_{0} \otimes e^{\lambda_{0}+\rho_{0}}\right)$.

Take the Bruhat filtration $\left\{I_{i}\right\}$ of $J_{\eta}^{\prime}(V)$. We will prove $I_{i} / I_{i-1}=0$ for all $i$. By (2), if $\eta$ is non-trivial on $w_{i} N_{0} w_{i}^{-1} \cap N_{0}$ then $I_{i} / I_{i-1}=0$. Hence we may assume that $\eta$ is not unitary on $w_{i} \overline{N_{0}} w_{i}^{-1} \cap N_{0}$. In this case, by the same argument as in the classical case (for example, see Schwartz's book [Sch66, Ch. VII, §4]), a nonzero element of $I_{i}^{\prime}$ is not tempered. Hence $I_{i} / I_{i-1}=0$.

Remark 3.7. In the next section it is proved that the conditions of Lemma 3.6 are also sufficient (Theorem 4.7).

Remark 3.8. If $\Pi=\operatorname{supp} \eta$, Lemma 3.6 follows from [CHM00, Theorem 5.12].

Definition 3.9 (Whittaker vectors). Let $V$ be a $U\left(\mathfrak{n}_{0}\right)$-module. We define a vector space $\mathrm{Wh}_{\eta}(V)$ by

$$
\mathrm{Wh}_{\eta}(V)=\left\{v \in V \mid X v=\eta(X) v \text { for all } X \in \mathfrak{n}_{0}\right\} .
$$

An element of $\mathrm{Wh}_{\eta}(V)$ is called a Whittaker vector.

Lemma 3.10. Assume that $\left.\eta\right|_{\operatorname{Ad}\left(w_{i}\right) \mathfrak{n} \cap \mathfrak{n}_{0}}=0$. Then

$$
\begin{aligned}
\mathrm{Wh}_{\eta}\left(\left\{\sum_{s}\left(f_{s} \eta_{i}^{-1} \otimes u_{s}^{\prime}\right) \delta_{i} \mid f_{s}\right.\right. & \left.\left.\in \mathcal{P}\left(O_{i}\right), u_{s}^{\prime} \in J_{w_{i}^{-1} \eta}^{\prime}\left(\sigma \otimes e^{\lambda+\rho}\right)\right\}\right) \\
& =\left\{\left(\eta_{i}^{-1} \otimes u^{\prime}\right) \delta_{i} \mid u^{\prime} \in \mathrm{Wh}_{w_{i}^{-1} \eta}\left(\sigma \otimes e^{\lambda+\rho}\right)\right\} .
\end{aligned}
$$

Proof. By assumption, we have $\eta=\eta^{\prime}$. Hence the right hand side is a subspace of the left hand side by Lemma 3.5.

Take $x=\sum_{s}\left(f_{s} \eta_{i}^{-1} \otimes u_{s}^{\prime}\right)=\sum_{s} \delta_{i}\left(1, f_{s} \eta_{i}^{-1}, u_{s}^{\prime}\right) \in \mathrm{Wh}_{\eta}\left(I_{i}^{\prime}\right)$. We assume that $\left\{u_{s}^{\prime}\right\}$ is linearly independent. Take $X \in \operatorname{Ad}\left(w_{i}\right) \overline{\mathfrak{n}} \cap \mathfrak{n}_{0}$. It then follows that $\sum_{s} \delta_{i}\left(1, L(X)\left(f_{s}\right) \eta_{i}^{-1}, u_{s}^{\prime}\right)=0$ by Lemma 3.5. Hence $L(X)\left(f_{s}\right)=0$. This implies $f_{s} \in \mathbb{C}$. 
From the above argument, $x=\delta_{i}\left(1, \eta_{i}^{-1}, u^{\prime}\right)$ for some $u^{\prime} \in J_{w_{i}^{-1} \eta}^{\prime}\left(\sigma \otimes e^{\lambda+\rho}\right)$. Take $X \in \operatorname{Ad}\left(w_{i}\right)\left(\mathfrak{m} \cap \mathfrak{n}_{0}\right)$. By Lemma 3.5 , we have

$$
\delta_{i}\left(1, \eta_{i}^{-1},\left(\operatorname{Ad}\left(w_{i}\right)^{-1} X-\eta(X)\right) u^{\prime}\right) \in \sum_{\mathbf{k} \neq 0, u_{\mathbf{k}} \in J_{w_{i}^{\prime} \eta}^{\prime}\left(\sigma \otimes e^{\lambda+\rho}\right)} \mathbb{C} \delta_{i}\left(1, f_{\mathbf{k}} \eta_{i}^{-1}, u_{\mathbf{k}}\right) .
$$

If $\mathbf{k} \neq 0$ then the degree of $f_{\mathbf{k}}$ is greater than 0 . So the left hand side must be 0 . Hence $\left(\operatorname{Ad}\left(w_{i}\right)^{-1} X-\eta(X)\right) u^{\prime}=0$, proving the lemma.

The following lemma is well-known, but we give a proof for the reader's convenience (cf. Casselman-Hecht-Miličić [CHM00], Yamashita [Yam86]).

Lemma 3.11. Assume that $\operatorname{supp} \eta=\Pi$.

(1) $\mathrm{Wh}_{\eta}\left(I(\sigma, \lambda)^{\prime}\right) \hookrightarrow \mathrm{Wh}_{\eta}\left(I_{r}^{\prime}\right)$, where the homomorphism is induced by $\operatorname{Res}_{r}$.

(2) For all $x \in \mathrm{Wh}_{\eta}\left(I_{r}^{\prime}\right)$, there exists $u^{\prime} \in \mathrm{Wh}_{w_{r}^{-1} \eta}\left(\left(\sigma \otimes e^{\lambda+\rho}\right)^{\prime}\right)$ such that $x=$ $\left(\eta_{r}^{-1} \otimes u^{\prime}\right) \delta_{r}$.

Recall that $r=\# W(M)=\#\left(W / W_{M}\right)$ and $w_{M, 0}$ is the longest element of the little Weyl group of $M$.

Proof. Assume that $i<r$. Then $w_{i} w_{M, 0}$ is not the longest element of $W$. There exists a simple root $\alpha \in \Pi$ such that $s_{\alpha} w_{i} w_{M, 0}>w_{i} w_{M, 0}$. This means that $w_{i} w_{M, 0} \Sigma^{+} \cap \Sigma^{+}=s_{\alpha}\left(s_{\alpha} w_{i} w_{M, 0} \Sigma^{+} \cap \Sigma^{+}\right) \cup\{\alpha\}$. The left hand side is $w_{i}\left(\Sigma^{+} \backslash\right.$ $\left.\Sigma_{M}^{+}\right) \cap \Sigma^{+}$. Hence, $\eta$ is not trivial on $\operatorname{Ad}\left(w_{i}\right) \mathfrak{n} \cap \mathfrak{n}_{0}$. By Lemma 3.6, $I_{i} / I_{i-1}=0$. This implies that $J_{\eta}^{\prime}(I(\sigma, \lambda)) \subset I_{r}^{\prime}$. Since $\operatorname{Ad}\left(w_{r}\right) \overline{\mathfrak{n}} \cap \overline{\mathfrak{n}_{0}}=0$, there exists a polynomial $f_{s} \in \mathcal{P}\left(O_{r}\right)$ and $u_{s}^{\prime} \in J_{w_{r}^{-1} \eta}^{\prime}\left(\sigma \otimes e^{\lambda+\rho}\right)$ such that $x=\sum_{s}\left(\left(f_{s} \eta_{r}^{-1}\right) \otimes u_{s}^{\prime}\right) \delta_{r}$. Now Lemma 3.10 yields the assertion.

\section{$\S 4$. Analytic continuation}

The aim of this section is to prove that $\operatorname{Im} \operatorname{Res}_{i}=I_{i}^{\prime}$ if $I_{i} / I_{i-1} \neq 0$. Namely, we extend an element of $I_{i}^{\prime}$ (which is a distribution on $U_{i}$ ) to $G / P$. An element of $I_{i}^{\prime}$ and $\varphi \in C_{c}^{\infty}\left(U_{i}\right)$ is given by an integral. Formally, this integral is valid for any $\varphi \in I(\sigma, \lambda)$. We prove the integral converges if $\lambda$ is sufficiently dominant. Moreover, as a function of $\lambda$, we prove this integral has a meromorphic continuation to $\mathfrak{a}^{*}$. (These are essentially known, but we give a proof for the sake of completeness.) The resulting distribution is a distribution on $G / P$ with a parameter $\lambda$. If it has no pole, this is an extension we need. In general, we can modify the distribution and remove the pole. This is the outline of the proof.

For $w \in W$, there is an open dense subset $w \bar{N} P / P$ of $G / P$ and it is diffeomorphic to $\bar{N}$. Then for $w, w^{\prime} \in W$, there exists a map $\Phi_{w, w^{\prime}}$ from some open 
dense subset $U \subset \bar{N}$ to $\bar{N}$ such that $w \bar{n} P / P=w^{\prime} \Phi_{w, w^{\prime}}(\bar{n}) P / P$ for $\bar{n} \in U$. The map $\Phi_{w, w^{\prime}}$ is a rational function.

Define $H: G \rightarrow \operatorname{Lie}(A)$ by $g \in K M \exp (H(g)) N$ via the Iwasawa decomposition.

Lemma 4.1. (1) The map $\bar{N} \rightarrow \mathbb{R}$ defined by $\bar{n} \mapsto e^{8 \rho(H(\bar{n}))}$ is a polynomial.

(2) For all $\bar{n} \in \bar{N}$ we have $e^{8 \rho(H(\bar{n}))} \geq 1$.

(3) Take $H_{0} \in \operatorname{Lie}(A)$ such that $\alpha\left(H_{0}\right)=-1$ for all $\alpha \in \Pi \backslash \Sigma_{M}$. There exists a continuous function $Q(\bar{n}) \geq 0$ on $\bar{N}$ such that the following conditions hold:

(a) The function $Q$ vanishes only at the unit element.

(b) $e^{8 \rho(H(\bar{n}))} \geq Q(\bar{n})$.

(c) $Q\left(\exp \left(t H_{0}\right) \bar{n} \exp \left(-t H_{0}\right)\right) \geq e^{8 t} Q(\bar{n})$ for $t \in \mathbb{R}_{>0}$ and $\bar{n} \in \bar{N}$.

Proof. By Knapp [Kna01, Proposition 7.19], there exists an irreducible finitedimensional representation $V_{4 \rho}$ of $G$ with highest weight $4 \rho \in \mathfrak{a}_{0}^{*} \subset \mathfrak{h}^{*}$. Let $v_{4 \rho} \in V_{4 \rho}$ be a highest weight vector and $v_{-4 \rho}^{*} \in V_{4 \rho}^{*}$ a lowest weight vector of $V_{4 \rho}^{*}$. Take $\bar{n} \in \bar{N}$ and decompose $\bar{n}=k a n$ where $k \in K, a \in A_{0}$ and $n \in N_{0}$. Then $\log (a) \in\left(\mathfrak{m} \cap \mathfrak{a}_{0}\right)+H(\bar{n})$. Hence $\rho(\log (a))=\rho(H(\bar{n}))$.

First we prove (1). We have $\theta(\bar{n})^{-1} \bar{n}=\theta(n)^{-1} a^{2} n$. Hence

$$
\begin{aligned}
\left\langle\theta(\bar{n})^{-1} \bar{n} v_{4 \rho}, v_{-4 \rho}^{*}\right\rangle & =\left\langle\theta(n)^{-1} a^{2} n v_{4 \rho}, v_{-4 \rho}^{*}\right\rangle=\left\langle a^{2} n v_{4 \rho}, \theta(n) v_{-4 \rho}^{*}\right\rangle \\
& =e^{8 \rho(H(\bar{n}))}\left\langle v_{4 \rho}, v_{-4 \rho}^{*}\right\rangle .
\end{aligned}
$$

The left hand side is a polynomial.

Next we prove (2) and (3). Fix a compact real form of $\mathfrak{g}$ containing Lie $(K)$ and take an inner product on $V_{4 \rho}$ which is invariant under the action of this compact real form. We normalize an inner product $\|\cdot\|$ so that $\left\|v_{4 \rho}\right\|=1$. Then $\left\|\bar{n} v_{4 \rho}\right\|=$ $\|$ kanv $_{4 \rho}\|=\| a v_{4 \rho}\left\|=e^{4 \rho(H(\bar{n}))}\right\| v_{4 \rho} \|=e^{4 \rho(H(\bar{n}))}$. For $\nu \in \mathfrak{h}^{*}$ let $Q_{\nu}(\bar{n}) \in V_{4 \rho}$ be a vector of weight $\nu$ such that $\bar{n} v_{4 \rho}=\sum_{\nu} Q_{\nu}(\bar{n})$. Then $e^{8 \rho(H(\bar{n}))}=\sum_{\nu}\left\|Q_{\nu}(\bar{n})\right\|^{2}$. Since $Q_{4 \rho}(\bar{n})=v_{4 \rho}$, we have $e^{8 \rho(H(\bar{n}))} \geq 1$.

Put $Q(\bar{n})=\sum_{w \in W(M) \backslash\{e\}}\left\|Q_{4 w \rho}(\bar{n})\right\|^{2}$. Assume that $\bar{n} \neq e$. Then there exist $w \in W(M) \backslash\{e\}, m^{\prime} \in M, a^{\prime} \in A, n^{\prime} \in N$ and $\bar{n}^{\prime} \in \bar{N}$ such that $\bar{n}=w \bar{n}^{\prime} m^{\prime} a^{\prime} n^{\prime}$. Let $v_{-4 w \rho}^{*} \in V_{4 \rho}^{*}$ be a weight vector with $\mathfrak{h}$-weight $-4 w \rho$ such that for all $v \in V_{4 w \rho}$, $\left|\left\langle v, v_{-4 w \rho}^{*}\right\rangle\right|=\|v\|$. Then

$$
\begin{aligned}
\left\|Q_{4 w \rho}(\bar{n})\right\| & =\left|\left\langle\bar{n} v_{4 \rho}, v_{-4 w \rho}^{*}\right\rangle\right|=\left|\left\langle w \bar{n}^{\prime} m^{\prime} a^{\prime} n^{\prime} v_{4 \rho}, v_{-4 w \rho}^{*}\right\rangle\right| \\
& =\left|\left\langle a^{\prime} v_{4 \rho}, w^{-1} v_{-4 w \rho}^{*}\right\rangle\right|=e^{4 \rho\left(\log a^{\prime}\right)}\left|\left\langle v_{4 \rho}, w^{-1} v_{-4 w \rho}^{*}\right\rangle\right| \neq 0 .
\end{aligned}
$$

Hence, if $\bar{n} \in \bar{N} \backslash\{e\}$ then $Q(\bar{n}) \neq 0$. 
Let $t>0$. Using $Q_{\nu}\left(\exp \left(t H_{0}\right) \bar{n} \exp \left(-t H_{0}\right)\right)=e^{t(\nu-4 \rho)\left(H_{0}\right)} Q_{\nu}(\bar{n})$, we have

$$
Q\left(\exp \left(t H_{0}\right) \bar{n} \exp \left(-t H_{0}\right)\right)=\sum_{w \in W(M) \backslash\{e\}} e^{8 t(w \rho-\rho)\left(H_{0}\right)}\left\|Q_{4 w \rho}(\bar{n})\right\|^{2} .
$$

Since $(w \rho-\rho)\left(H_{0}\right) \geq 1$ for $w \in W(M) \backslash\{e\}$, we get the lemma.

Remark 4.2. The conditions in Lemma 4.1(3) imply that $\lim _{\bar{n} \rightarrow \infty} Q(\bar{n})=\infty$. Indeed, take $H_{0}$ as in Lemma 4.1. Let $\left\{e_{1}, \ldots, e_{l}\right\}$ be a basis of $\overline{\mathfrak{n}}$. We assume that each $e_{s}$ is a restricted root vector and denote its root by $\alpha_{s}$. Any $\bar{n} \in \bar{N}$ can be written as $\bar{n}=\exp \left(\sum_{s=1}^{l} a_{s} e_{s}\right)$ where $a_{s} \in \mathbb{R}$. We have $\alpha_{s}\left(H_{0}\right)>0$ for all $s=$ $1, \ldots, l$. Put $r(\bar{n})=\sum_{s=1}^{l}\left|a_{s}\right|^{1 / \alpha_{s}\left(H_{0}\right)}$. Set $C=\min _{r(\bar{n})=1} Q(\bar{n})$. Since $Q(\bar{n})>0$ if $\bar{n}$ is not the unit element, $C>0$. Put $t=\log r(\bar{n})$ and set $\bar{n}^{\prime}=\exp \left(-t H_{0}\right) \bar{n} \exp \left(t H_{0}\right)$. Then $\bar{n}^{\prime}=\exp \left(\sum_{s=1}^{l} a_{s} e^{-t \alpha_{s}\left(H_{0}\right)} e_{s}\right)$. Therefore, $r\left(\bar{n}^{\prime}\right)=\sum_{s=1}^{l}\left|a_{s}\right|^{1 / \alpha_{s}\left(H_{0}\right)} e^{-t}=1$. Hence, if $r(\bar{n})>1$, then $Q(\bar{n})=Q\left(\exp \left(t H_{0}\right) \bar{n}^{\prime} \exp \left(-t H_{0}\right)\right) \geq C e^{8 t}=C r(\bar{n})^{8}$ by Lemma 4.1(3). If $\bar{n} \rightarrow \infty$ then $r(\bar{n}) \rightarrow \infty$. Hence, $Q(\bar{n}) \rightarrow \infty$.

Lemma 4.3. Let $f$ be a polynomial on $\bar{N}$. There exists a positive integer $k$ such that a $C^{\infty}$-function $h$ on $w_{i} \bar{N} P / P$ defined by $h\left(w_{i} \bar{n} P / P\right)=e^{-k \rho(H(\bar{n}))} f(\bar{n})$ can be extended to a $C^{\infty}$-function on $G / P$.

Proof. By Lemma 4.1 and Remark 4.2, we can choose a positive integer $k$ such that $\lim _{\bar{n} \rightarrow \infty} e^{-8 k \rho(H(\bar{n}))} f(\bar{n})=0$. Let $h$ be a function on $U_{i}$ defined by $h\left(w_{i} \bar{n} P / P\right)=$ $e^{-8 k \rho(H(\bar{n}))} f(\bar{n})$ for $\bar{n} \in \bar{N}$. We prove that $h$ can be extended to $G / P$ as a $C^{\infty}$ function. Take $w \in W(M)$. Then $h$ is defined on a subset of $w \bar{N} P / P$. Using a diffeomorphism $\bar{N} \simeq w \bar{N} P / P, h$ defines a rational function $h \circ \Phi_{w_{i}, w}$ defined on an open dense subset of $\bar{N}$. By the condition on $k$, the function $h \circ \Phi_{w_{i}, w}$ has no pole. Hence, $h$ defines a $C^{\infty}$-function on $w \bar{N} P / P$. Since $\bigcup_{w \in W(M)} w \bar{N} P / P=G / P$, the lemma follows.

Recall that for a representation $V$ of $\mathfrak{m}, \nu \in\left(\mathfrak{m} \cap \mathfrak{a}_{0}\right)^{*} \subset \mathfrak{a}_{0}^{*}$ is called an exponent of $V$ if $\nu+\left.\rho_{0}\right|_{\mathfrak{m} \cap \mathfrak{a}_{0}}$ is an $\mathfrak{a}_{0}$-weight of $V /\left(\mathfrak{m} \cap \mathfrak{n}_{0}\right) V$.

Proposition 4.4. Let $\varphi$ be a $\sigma$-valued function on $K$ which satisfies $\varphi(k m)=$ $\sigma(m)^{-1} \varphi(k)$ for all $k \in K$ and $m \in M \cap K$. Define $\varphi_{\lambda} \in I(\sigma, \lambda)$ by $\varphi_{\lambda}($ kman $)=$ $e^{-(\lambda+\rho)(\log a)} \sigma(m)^{-1} \varphi(k)$ for $k \in K, m \in M, a \in A$ and $n \in N$. For $u^{\prime} \in$ $J_{w_{i}^{-1} \eta}^{\prime}\left(\sigma \otimes e^{\lambda+\rho}\right)$ and $f \in \mathcal{P}\left(O_{i}\right)$, put

$$
I_{f, u^{\prime}}\left(\varphi_{\lambda}\right)=\int_{w_{i} \bar{N} w_{i}^{-1} \cap N_{0}} u^{\prime}\left(\varphi_{\lambda}\left(n w_{i}\right)\right) \eta(n)^{-1} f\left(n w_{i}\right) d n .
$$

(1) If $\langle\check{\alpha}, \operatorname{Re} \lambda\rangle$ is sufficiently large for each $\alpha \in \Sigma^{+} \backslash \Sigma_{M}^{+}$then the integral $I_{f, u^{\prime}}\left(\varphi_{\lambda}\right)$ absolutely converges. 
(2) As a function of $\lambda$, the integral $I_{f, u^{\prime}}\left(\varphi_{\lambda}\right)$ has a meromorphic continuation to $\mathfrak{a}^{*}$.

(3) If $\operatorname{supp} \eta=\Pi$ and $i=r$ then $I_{f, u^{\prime}}\left(\varphi_{\lambda}\right)$ is holomorphic at any $\lambda \in \mathfrak{a}^{*}$.

(4) Let $u^{\prime} \in \mathrm{Wh}_{w_{i}^{-1} \eta}\left(\left(\sigma \otimes e^{\lambda+\rho}\right)^{\prime}\right)$. If $\langle\check{\alpha}, \lambda+\nu\rangle \notin \mathbb{Z}_{\leq 0}$ for all exponents $\nu$ of $\sigma$ and $\alpha \in \Sigma^{+} \backslash w_{i}^{-1}\left(\Sigma^{+} \cup \Sigma_{\eta}^{-}\right)$, then $I_{1, u^{\prime}}\left(\varphi_{\mu}\right)$ is holomorphic at $\mu=\lambda$.

For a proof, we use the following notation. (It will also be used in Sections 7 and 8.)

Let $P_{\eta} \supset P_{0}$ be the parabolic subgroup corresponding to $\operatorname{supp} \eta \subset \Pi$ and $P_{\eta}=M_{\eta} A_{\eta} N_{\eta}$ its Langlands decomposition such that $A_{\eta} \subset A_{0}$. Denote the complexifications of the Lie algebras of $P_{\eta}, M_{\eta}, A_{\eta}, N_{\eta}$ by $\mathfrak{p}_{\eta}, \mathfrak{m}_{\eta}, \mathfrak{a}_{\eta}, \mathfrak{n}_{\eta}$, respectively. Put $\mathfrak{l}_{\eta}=\mathfrak{m}_{\eta} \oplus \mathfrak{a}_{\eta}, \overline{N_{\eta}}=\theta\left(N_{\eta}\right)$ and $\overline{\mathfrak{n}_{\eta}}=\theta\left(\mathfrak{n}_{\eta}\right)$. Set $\Sigma_{\eta}^{+}=\left\{\sum_{\alpha \in \operatorname{supp} \eta} n_{\alpha} \alpha \in \Sigma^{+} \mid\right.$ $\left.n_{\alpha} \in \mathbb{Z}_{\geq 0}\right\}$ and $\Sigma_{\eta}^{-}=-\Sigma_{\eta}^{+}$.

Proof. First we prove (1). If $f=1$ then this is a well-known result. (See, for example, Knapp's book [Kna01, Theorem 7.22].) For a general $f$, extend $f$ to a function on $w_{i} \bar{N} P / P$ by $f\left(n n^{\prime} w_{i}\right)=f\left(n w_{i}\right)$ for $n \in w_{i} \bar{N} w_{i}^{-1} \cap N_{0}$ and $n^{\prime} \in$ $w_{i} \bar{N} w_{i}^{-1} \cap \overline{N_{0}}$. Then by Lemma 4.3 there exists a positive integer $C$ such that $\bar{n} \mapsto e^{-C \rho(H(\bar{n}))} f\left(w_{i} \bar{n}\right)$ extends to a $C^{\infty}$-function $h$ on $G / P$. Define $\kappa: G \rightarrow K$ by $g \in \kappa(g) A_{0} N_{0}$. Since

$$
I_{f, u^{\prime}}\left(\varphi_{\lambda}\right)=\int_{w_{i} \bar{N} w_{i}^{-1} \cap N_{0}} u^{\prime}(\varphi(\kappa(n w))) e^{-(\lambda+\rho)\left(H\left(n w_{r}\right)\right)} f\left(n w_{r}\right) \eta(n)^{-1} d n,
$$

we have $I_{f, u^{\prime}}\left(\varphi_{\lambda}\right)=I_{1, u^{\prime}}\left((\varphi h)_{\lambda-C \rho}\right)$.

We prove (3). By dualizing Casselman's subrepresentation theorem, there exist an irreducible representation $\sigma_{0}$ of $M_{0}$ and $\lambda_{0} \in \mathfrak{a}_{0}^{*}$ such that $\sigma$ is a quotient of $\operatorname{Ind}_{M \cap P_{0}}^{M}\left(\sigma_{0} \otimes e^{\lambda_{0}}\right)$. We may regard $u^{\prime} \in J_{w_{r}^{-1} \eta}^{\prime}\left(\operatorname{Ind}_{M \cap P_{0}}^{M}\left(\sigma_{0} \otimes e^{\lambda_{0}}\right)\right)$. By the proof of Lemma 3.11, there exist a polynomial $f_{0}$ on $\left(M \cap N_{0}\right) w_{M, 0}\left(M \cap P_{0}\right) /\left(M \cap P_{0}\right)$ and $u_{0}^{\prime} \in\left(\sigma_{0} \otimes e^{\lambda_{0}}\right)^{\prime}$ such that $u^{\prime}$ is given by

$$
\varphi_{0} \mapsto \int_{M \cap N_{0}} u_{0}^{\prime}\left(\varphi_{0}\left(n_{0} w_{M, 0}\right)\right) f_{0}\left(n_{0} w_{M, 0}\right) \eta\left(n_{0}\right)^{-1} d n_{0}
$$

Let $\pi: \operatorname{Ind}_{P_{0}}^{G}\left(\sigma_{0} \otimes e^{\lambda+\lambda_{0}+\rho}\right) \rightarrow I(\sigma, \lambda)$ be the map induced from the quotient map $\operatorname{Ind}_{M \cap P_{0}}^{M}\left(\sigma_{0} \otimes e^{\lambda_{0}}\right) \rightarrow \sigma$. Take $\widetilde{\varphi}: K \rightarrow \sigma_{0}$ with $\tilde{\varphi}(k m)=\sigma_{0}^{-1}(m) \widetilde{\varphi}(k)(k \in K$, $\left.m \in M_{0}\right)$ and $\pi\left(\widetilde{\varphi}_{\lambda+\lambda_{0}}\right)=\varphi_{\lambda}$. Define a polynomial $\widetilde{f} \in \mathcal{P}\left(w_{r} w_{M, 0} \overline{N_{0}} P_{0} / P_{0}\right)$ by

$$
\widetilde{f}\left(w_{r} w_{M, 0} n n_{0} P_{0} / P_{0}\right)=f\left(w_{r} n P / P\right) f_{0}\left(w_{M, 0} n_{0}\left(M \cap P_{0}\right) /\left(M \cap P_{0}\right)\right)
$$

for $n \in \bar{N}$ and $n_{0} \in M \cap \overline{N_{0}}$. (Notice that $w_{M, 0}\left(M \cap \overline{N_{0}}\right)=\left(M \cap N_{0}\right) w_{M, 0}$, so $f$ 
is a polynomial on $w_{M, 0}\left(M \cap \overline{N_{0}}\right)\left(M \cap P_{0}\right) /\left(M \cap P_{0}\right)$.) We have

$$
\begin{aligned}
I_{f, u^{\prime}}\left(\varphi_{\lambda}\right)=\int_{w_{r} w_{M, 0} \overline{N_{0}}\left(w_{r} w_{M, 0}\right)^{-1} \cap N_{0}} u_{0}^{\prime}\left(\widetilde{\varphi}_{\lambda+\lambda_{0}}\left(n w_{r} w_{M, 0}\right)\right) \\
\times \widetilde{f}\left(w_{r} w_{M, 0} n P_{0} / P_{0}\right) \eta(n)^{-1} d n .
\end{aligned}
$$

Hence, we may assume that $P$ is minimal. By the same argument as in the proof of (1), we may assume $f=1$. If $f=1$ then this integral is known as a Jacquet integral and its analytic continuation is known [Jac67].

We prove (2) and (4). By the same argument in the proof of (1), we may assume that $f=1$. Using Casselman's subrepresentation theorem, there exist an irreducible representation $\sigma_{0}$ of $M_{0}, \nu \in \mathfrak{a}_{0}^{*}$ and a surjective homomorphism $\operatorname{Ind}_{P_{0}}^{G}\left(\sigma_{0} \otimes e^{\lambda+\nu+\rho_{0}}\right) \rightarrow I(\sigma, \lambda)$. Moreover, $\nu$ is an exponent of $\sigma$. By the same argument as in the proof of (3), we may assume $P=P_{0}$. (Hence each exponent of $\sigma$ is 0 .)

Take $w^{\prime} \in W_{M_{\eta}}$ and $w^{\prime \prime} \in W\left(M_{\eta}\right)^{-1}$ such that $w_{i}=w^{\prime} w^{\prime \prime}$. Then we have $w_{i} \bar{N} w_{i}^{-1} \cap N_{0}=w_{i} \overline{N_{0}} w_{i}^{-1} \cap N_{0}=\left(w^{\prime} \overline{N_{0}}\left(w^{\prime}\right)^{-1} \cap N_{0}\right) w^{\prime}\left(w^{\prime \prime} \overline{N_{0}}\left(w^{\prime \prime}\right)^{-1} \cap N_{0}\right)\left(w^{\prime}\right)^{-1}$. The condition $w^{\prime} \in W_{M_{\eta}}$ implies that $w^{\prime}\left(\Sigma^{+} \backslash \Sigma_{\eta}^{+}\right)=\Sigma^{+} \backslash \Sigma_{\eta}^{+}$. Hence, supp $\eta \cap w^{\prime} \Sigma^{+}$ $=\operatorname{supp} \eta \cap w^{\prime} \Sigma_{\eta}^{+}$. This implies

$$
\begin{aligned}
& \operatorname{supp} \eta \cap w^{\prime}\left(w^{\prime \prime} \Sigma^{-} \cap \Sigma^{+}\right)=\operatorname{supp} \eta \cap w_{i} \Sigma^{-} \cap w^{\prime} \Sigma^{+} \\
& =\operatorname{supp} \eta \cap w_{i} \Sigma^{-} \cap w_{i}\left(w^{\prime \prime}\right)^{-1} \Sigma_{\eta}^{+} \subset \operatorname{supp} \eta \cap w_{i} \Sigma^{-} \cap w_{i} \Sigma^{+}=\emptyset,
\end{aligned}
$$

i.e., $\eta$ is trivial on $w^{\prime}\left(w^{\prime \prime} \overline{N_{0}}\left(w^{\prime \prime}\right)^{-1} \cap N_{0}\right)\left(w^{\prime}\right)^{-1}$. Hence

$$
I_{1, u^{\prime}}(\varphi)=\int_{w^{\prime} \overline{N_{0}}\left(w^{\prime}\right)^{-1} \cap N_{0}} \int_{w^{\prime \prime} \overline{N_{0}}\left(w^{\prime \prime}\right)^{-1} \cap N_{0}} u^{\prime}\left(\varphi\left(n_{1} w^{\prime} n_{2} w^{\prime \prime}\right)\right) \eta\left(n_{1}\right)^{-1} d n_{2} d n_{1} .
$$

Define a $G$-module homomorphism $A(\sigma, \lambda): I(\sigma, \lambda) \rightarrow \operatorname{Ind}_{P_{0}}^{G}\left(w^{\prime \prime}(\sigma) \otimes e^{w^{\prime \prime} \lambda+\rho_{0}}\right)$ by

$$
(A(\sigma, \lambda) \psi)(x)=\int_{w^{\prime \prime} \overline{N_{0}}\left(w^{\prime \prime}\right)^{-1} \cap N_{0}} \psi\left(x n w^{\prime \prime}\right) d n .
$$

By a result of Knapp and Stein [KS80], this homomorphism has a meromorphic continuation. We have

$$
I_{1, u^{\prime}}(\psi)=\int_{w^{\prime} \overline{N_{0}}\left(w^{\prime}\right)^{-1} \cap N_{0}} u^{\prime}\left((A(\sigma, \lambda) \psi)\left(n w^{\prime}\right)\right) \eta(n)^{-1} d n
$$

Notice that $w^{\prime} \overline{N_{0}}\left(w^{\prime}\right)^{-1} \cap N_{0} \subset M_{\eta}$. Hence $I_{1, u^{\prime}}$ is given by the composition

$$
\begin{aligned}
I(\sigma, \lambda) \stackrel{A(\sigma, \lambda)}{\longrightarrow} \operatorname{Ind}_{P_{0}}^{G}( & \left.w^{\prime \prime}(\sigma) \otimes e^{w^{\prime \prime} \lambda+\rho_{0}}\right) \\
& \stackrel{\text { restriction to } M_{\eta}}{\longrightarrow} \operatorname{Ind}_{M_{\eta} \cap P_{0}}^{M_{\eta}}\left(w^{\prime \prime}(\sigma) \otimes e^{w^{\prime \prime} \lambda+\rho_{0}}\right) \rightarrow \mathbb{C} .
\end{aligned}
$$


Here the last map is given by

$$
\psi \mapsto \int_{w^{\prime} \overline{N_{0}} w^{\prime-1} \cap N_{0}} u^{\prime}\left(\psi\left(n w^{\prime}\right)\right) \eta(n)^{-1} d n .
$$

By (3), this integral is holomorphic. Hence we get (2).

To prove (4), we calculate $\left(w^{\prime \prime}\right)^{-1} \Sigma^{-} \cap \Sigma^{+}$. Since $\left(w^{\prime \prime}\right)^{-1} \in W\left(M_{\eta}\right)$, we have $\left(w^{\prime \prime}\right)^{-1} \Sigma_{\eta}^{-} \subset \Sigma^{-}$. Hence $\left(w^{\prime \prime}\right)^{-1} \Sigma_{\eta}^{-} \cap \Sigma^{+}=\emptyset$. Then

$$
\begin{aligned}
\left(w^{\prime \prime}\right)^{-1} \Sigma^{-} \cap \Sigma^{+} & =\left(w^{\prime \prime}\right)^{-1}\left(\Sigma^{-} \backslash \Sigma_{\eta}^{-}\right) \cap \Sigma^{+}=\left(w^{\prime \prime}\right)^{-1}\left(w^{\prime}\right)^{-1}\left(\Sigma^{-} \backslash \Sigma_{\eta}^{-}\right) \cap \Sigma^{+} \\
& =w_{i}^{-1}\left(\Sigma^{-} \backslash \Sigma_{\eta}^{-}\right) \cap \Sigma^{+}=\Sigma^{+} \backslash w_{i}^{-1}\left(\Sigma^{+} \cup \Sigma_{\eta}^{-}\right) .
\end{aligned}
$$

Hence $\langle\check{\alpha}, \lambda\rangle \notin \mathbb{Z}_{\geq 0}$ for all $\alpha \in\left(w^{\prime \prime}\right)^{-1} \Sigma^{-} \cap \Sigma^{+}$. By an argument of Knapp and Stein [KS80], $A(\sigma, \mu)$ is holomorphic at $\mu=\lambda$ if $\lambda$ satisfies the conditions of (4). Hence we get (4).

In the rest of this section, we denote the Bruhat filtration $I_{i} \subset J_{\eta}^{\prime}(I(\sigma, \lambda))$ by $I_{i}(\lambda)$. The following result is a corollary of Proposition 4.4.

Lemma 4.5. Let $x \in I_{i}^{\prime}$. Then there exists a distribution $x_{t} \in I_{i}(\lambda+t \rho)$ with a meromorphic parameter $t$ such that $\left.x_{t}\right|_{U_{i}}$ is a distribution with a holomorphic parametert and $\left.\left(\left.x_{t}\right|_{U_{i}}\right)\right|_{t=0}=x$. Moreover, for $E \in U(\mathfrak{g}), E x=0$ implies $E x_{t}=0$.

Proof. By the definition of $I_{i}^{\prime}$, we may assume $x=E\left(\left(f \eta_{i}^{-1} \otimes u^{\prime}\right) \delta_{i}\right)$ for some $E \in U\left(\operatorname{Ad}\left(w_{i}\right) \overline{\mathfrak{n}} \cap \overline{\mathfrak{n}_{0}}\right), f \in \mathcal{P}\left(O_{i}\right)$ and $u^{\prime} \in J_{w_{i}^{-1} \eta}^{\prime}\left(\sigma \otimes e^{\lambda+\rho}\right)$. By (1) and (2) of the above proposition, $\varphi \mapsto I_{f, u^{\prime}}\left(\varphi_{\lambda+t \rho}\right)$ is a distribution with a meromorphic parameter $t$. Moreover, it does not have a pole near $t=0$ by (4) of the proposition. Let $x_{t}^{\prime}$ be this distribution. Put $x_{t}=E x_{t}^{\prime}$. By construction, $x_{t}$ is as desired.

Let $C^{\infty}(K, \sigma)$ be the space of $\sigma$-valued $C^{\infty}$-functions on $K$. For $X \in \mathfrak{g}$ and $\lambda \in \mathfrak{a}^{*}$, we define an operator $D(X, \lambda)$ on $C^{\infty}(K, \sigma)$ by

$$
(D(X, \lambda) \varphi)(k)=\left.\frac{d}{d t}\left(\sigma \otimes e^{\lambda+\rho}\right)(\exp (-H(\exp (-t X) k))) \varphi(\kappa(\exp (-t X) k))\right|_{t=0}
$$

for $\varphi \in C^{\infty}(K, \sigma)$. If we regard $I(\sigma, \lambda)$ as a subspace of $C^{\infty}(K, \sigma)$, then $(X \varphi)(k)=$ $(D(X, \lambda) \varphi)(k)$ for $\varphi \in I(\sigma, \lambda)$. It is easy to see that there exist differential operators $D_{1}, D_{2}$ on $\varphi$ such that $D(X, \lambda+t \rho)=D_{1}+t D_{2}$ for all $t \in \mathbb{C}$. (The operators $D_{1}, D_{2}$ may depend on $X$ and $\lambda$, but do not depend on $t$.)

Lemma 4.6. Assume that the following conditions hold.

(1) The character $\eta$ is unitary. 
(2) The character $\eta$ is zero on $\operatorname{Ad}\left(w_{i}\right) \mathfrak{n} \cap \mathfrak{n}_{0}$.

(3) The module $J_{w_{i}^{-1} \eta}^{\prime}\left(\sigma \otimes e^{\lambda+\rho}\right)$ is not zero.

(See Lemma 3.6.) For $x \in I_{i}^{\prime}$ there exists a distribution $x_{t} \in I_{i}(\lambda+t \rho)$ with a holomorphic parameter $t$ defined near $t=0$ such that $x_{0}=x$ on $U_{i}$.

Proof. First we remark that $\eta=\eta^{\prime}$ by (2).

We argue by induction on $i$. If $i=1$, then $x \in I_{1}^{\prime}$. Take a distribution $x_{t} \in I_{1}(\lambda+t \rho)$ as in Lemma 4.5. Then $\left.x_{t}\right|_{U_{1}}$ is holomorphic with respect to $t$. Since $\operatorname{supp} x_{t} \subset O_{1},\left.x_{t}\right|_{(G / P) \backslash O_{1}}$ is holomorphic with respect to $t$. Hence $x_{t}$ is holomorphic with respect to $t$ on $U_{1} \cup\left((G / P) \backslash O_{1}\right)=G / P$ as desired.

Assume that $i>1$. First we prove the following claim: for $y \in I_{i-1}(\lambda)$, there exists a distribution $y_{t} \in I_{i-1}(\lambda+t \rho)$ with a holomorphic parameter $t$ defined near $t=0$ such that $y_{0}=y$. Applying the inductive hypothesis to $\left.y\right|_{U_{i-1}}$, there exists a distribution $y_{t}^{(i-1)} \in I_{i-1}(\lambda+t \rho)$ with a holomorphic parameter $t$ defined near $t=0$ such that $y_{0}^{(i-1)}=y$ on $U_{i-1}$. Since the supports of both sides are contained in $\bigcup_{j \leq i-1} N_{0} w_{j} P / P$, we have $y_{0}^{(i-1)}=y$ on $\bigcup_{j \geq i-1} N_{0} w_{j} P / P$. Applying the inductive hypothesis to $\left.\left(y-y_{0}^{(i-1)}\right)\right|_{U_{i-2}}$, there exists a distribution $y_{t}^{(i-2)} \in I_{i-2}(\lambda+t \rho)$ with a holomorphic parameter $t$ defined near $t=0$ such that $y_{0}^{(i-2)}=y-y_{0}^{(i-1)}$ on $U_{i-2}$. Since the supports of both sides are contained in $\bigcup_{j \leq i-2} N_{0} w_{j} P / P$, we have $y_{0}^{(i-1)}+y_{0}^{(i-2)}=y$ on $\bigcup_{j \geq i-2} N_{0} w_{j} P / P$. Iterating this argument, for $j=1, \ldots, i-1$ there exists a distribution $y_{t}^{(j)} \in I_{j}(\lambda+t \rho)$ with a holomorphic parameter $t$ defined near $t=0$ such that $y=y_{0}^{(1)}+\cdots+y_{0}^{(i-1)}$ on $G / P$. Hence we get the claim.

Now we prove the lemma. By Lemma 4.5, there exists a distribution $x_{t}^{\prime} \in$ $I_{i}(\lambda+t \rho)$ with a meromorphic parameter $t$ such that $\left.x_{t}^{\prime}\right|_{U_{i}}$ is holomorphic and $\left.\left(\left.x_{t}^{\prime}\right|_{U_{i}}\right)\right|_{t=0}=x$. Let $x_{t}^{\prime}=\sum_{s=-p}^{\infty} x^{(s)} t^{s}$ be the Laurent series of $x_{t}^{\prime}$. Now we prove the following claim: if there exists a distribution $x_{t}^{\prime}=\sum_{s=-p}^{\infty} x^{(s)} t^{s} \in I_{i}(\lambda+t \rho)$ with a meromorphic parameter $t$ defined near $t=0$ such that $\left.x_{t}^{\prime}\right|_{U_{i}}$ is holomorphic and $\left.\left(\left.x_{t}^{\prime}\right|_{U_{i}}\right)\right|_{t=0}=x$, then there exists $x_{t} \in I_{i}(\lambda+t \rho)$ with a holomorphic parameter $t$ defined near $t=0$ such that $\left.x_{0}\right|_{U_{i}}=x$. We prove the claim by induction on $p$.

If $p=0$, we have nothing to prove. Assume $p>0$. Take $E \in \mathfrak{n}_{0}$ and define differential operators $E_{0}$ and $E_{1}$ by $D(E, \lambda+t \rho)=E_{0}+t E_{1}$. There exists a positive integer $k$ such that $\left(E_{0}+t E_{1}-\eta(E)\right)^{k} x_{t}^{\prime}=0$. Hence $\left(E_{0}-\eta(E)\right)^{k} x^{(-p)}=0$. Since $\left.x_{t}\right|_{U_{i}}$ is holomorphic, $\operatorname{supp} x^{(-p)} \subset \bigcup_{j<i} N_{0} w_{j} P / P$. Hence $x^{(-p)} \in I_{i-1}(\lambda)$. By the claim in the third paragraph of this proof, there exists $x_{t}^{\prime \prime} \in I_{i-1}(\lambda+t \rho)$ with a holomorphic parameter $t$ defined near $t=0$ such that $x_{0}^{\prime \prime}=x^{(-p)}$. Using the inductive hypothesis for $x_{t}^{\prime}-t^{-p} x_{t}^{\prime \prime}$, we get the claim and the assertion of the lemma. 
Theorem 4.7. (1) The module $I_{i} / I_{i-1}$ is non-zero if and only if the following conditions hold:

(a) The character $\eta$ is unitary.

(b) The character $\eta$ is zero on $\operatorname{Ad}\left(w_{i}\right) \mathfrak{n} \cap \mathfrak{n}_{0}$.

(c) The module $J_{w_{i}^{-1} \eta}^{\prime}\left(\sigma \otimes e^{\lambda+\rho}\right)$ is not zero.

(2) If $I_{i} / I_{i-1} \neq 0$ then $I_{i} / I_{i-1} \simeq I_{i}^{\prime}$.

Proof. Assume that conditions (a)-(c) hold. We prove that the homomorphism $\operatorname{Res}_{i}: I_{i} \rightarrow I_{i}^{\prime}$ defined in Section 2 is surjective. Indeed, for $x \in I_{i}^{\prime}$, take $x_{t} \in$ $I_{i}(\lambda+t \rho)$ as in Lemma 4.6. Then $\operatorname{Res}_{i}\left(x_{0}\right)=\left.\left(x_{0}\right)\right|_{U_{i}}=x$.

\section{$\S 5$. Twisting functors}

Arkhipov defined the twisting functor for $\widetilde{w} \in \widetilde{W}$ [Ark04]. In this section, we define a modification of the twisting functor.

Let $\mathfrak{g}_{\alpha}^{\mathfrak{h}}$ be the root space of $\alpha \in \Delta$. Set $\mathfrak{u}_{0}=\bigoplus_{\alpha \in \Delta^{+}} \mathfrak{g}_{\alpha}^{\mathfrak{h}}, \overline{\mathfrak{u}_{0}}=\bigoplus_{\alpha \in \Delta^{+}} \mathfrak{g}_{-\alpha}^{\mathfrak{h}}$ and $\mathfrak{u}_{0, \widetilde{w}}=\operatorname{Ad}(\widetilde{w}) \overline{\mathfrak{u}_{0}} \cap \mathfrak{u}_{0}$. Let $\psi$ be a character of $\mathfrak{u}_{0, \widetilde{w}}$. Let $\left\{e_{1}, \ldots, e_{l}\right\}$ be a basis of $\mathfrak{u}_{0, \widetilde{w}}$ such that each $e_{i}$ is a root vector and $\bigoplus_{s \leq t-1} \mathbb{C} e_{s}$ is an ideal of $\bigoplus_{s \leq t} \mathbb{C} e_{s}$ for each $t=1, \ldots, l$. Notice that the multiplicative set $\left\{\left(e_{k}-\psi\left(e_{k}\right)\right)^{n} \mid n \in \mathbb{Z}_{\geq 0}\right\}$ satisfies the Ore condition for $k=1, \ldots, l$. Then we can consider the localization of $U(\mathfrak{g})$ with respect to $\left\{\left(e_{k}-\psi\left(e_{k}\right)\right)^{n} \mid n \in \mathbb{Z}_{\geq 0}\right\}$. We denote the resulting algebra by $U(\mathfrak{g})_{e_{k}-\psi\left(e_{k}\right)}$. Put $S_{e_{k}-\psi\left(e_{k}\right)}=U(\mathfrak{g})_{e_{k}-\psi\left(e_{k}\right)} / U(\mathfrak{g})$. Then $S_{e_{k}-\psi\left(e_{k}\right)}$ is a $U(\mathfrak{g})-$ bimodule.

Proposition 5.1. The $U(\mathfrak{g})$-bimodule structure on

$$
S_{e_{1}-\psi\left(e_{1}\right)} \otimes_{U(\mathfrak{g})} \cdots \otimes_{U(\mathfrak{g})} S_{e_{l}-\psi\left(e_{l}\right)}
$$

is independent of the choice of $e_{1}, \ldots, e_{l}$.

We denote this module by $S_{\widetilde{w}, \psi}$.

The proof of this proposition is similar to that of [Ark04, Theorem 2.1.6]. We omit it. Every element of $S_{\widetilde{w}, \psi}$ can be written as a sum of elements of the form $\left(e_{1}-\psi\left(e_{1}\right)\right)^{-\left(k_{1}+1\right)} \otimes \cdots \otimes\left(e_{l}-\psi\left(e_{l}\right)\right)^{-\left(k_{l}+1\right)} E$ for $E \in U(\mathfrak{g})$. We denote this element by $\left(e_{1}-\psi\left(e_{1}\right)\right)^{-\left(k_{1}+1\right)} \cdots\left(e_{l}-\psi\left(e_{l}\right)\right)^{-\left(k_{l}+1\right)} E$ for short.

For any $U(\mathfrak{g})$-module $V$, we now define a $U(\mathfrak{g})$-module $T_{\widetilde{w}, \psi} V$ by $T_{\widetilde{w}, \psi} V=$ $S_{\widetilde{w}, \psi} \otimes_{U(\mathfrak{g})}(\widetilde{w} V)$. (Recall that $\widetilde{w} V$ is a $\mathfrak{g}$-module twisted by $\widetilde{w}$, see Notation.) This gives the twisting functor $T_{\widetilde{w}, \psi}$. This is an endo-functor of the category of $\mathfrak{g}$-modules. If $\psi$ is the trivial representation, $T_{\widetilde{w}, \psi}$ is the twisting functor defined by Arkhipov. We put $T_{\widetilde{w}}=T_{\widetilde{w}, 0}$ where 0 is the trivial representation of $\mathfrak{u}_{0, \widetilde{w}}$. 
Remark 5.2. Arkhipov [Ark04] denotes the twisting functor by $\Theta_{w}$. We follow the notation of Andersen-Lauritzen [AL03].

We have a natural homomorphism $N_{K}(\mathfrak{h}) / Z_{K}(\mathfrak{h}) \rightarrow N_{K}\left(\mathfrak{a}_{0}\right) / Z_{K}\left(\mathfrak{a}_{0}\right)=W$.

Lemma 5.3. Let $w \in W$. Then there exists $\iota(w) \in N_{K}(\mathfrak{h})$ such that $\left.\operatorname{Ad}(\iota(w))\right|_{\mathfrak{a}_{0}}$ $=w$ and $\operatorname{Ad}(\iota(w))\left(\Delta_{M_{0}}^{+}\right)=\Delta_{M_{0}}^{+}$. If $\iota(w)$ and $\iota(w)^{\prime}$ both satisfy these conditions, then $\iota(w) \in \iota(w)^{\prime} Z_{K}(\mathfrak{h})$.

Proof. Since $W=N_{K}\left(\mathfrak{a}_{0}\right) / Z_{K}\left(\mathfrak{a}_{0}\right)$, there is $k \in N_{K}\left(\mathfrak{a}_{0}\right)$ such that $\left.\operatorname{Ad}(k)\right|_{\mathfrak{a}_{0}}=w$. Then $k$ normalizes $M_{0}$. Hence there exists $m \in M_{0}$ such that $k m$ normalizes $T_{0}$. This implies $k m \in N_{K}\left(A_{0} T_{0}\right)$. Take $w^{\prime} \in N_{M_{0}}\left(\mathfrak{t}_{0}\right)$ such that $\operatorname{Ad}\left(k m w^{\prime}\right)\left(\Delta_{M_{0}}^{+}\right)=$ $\Delta_{M_{0}}^{+}$and put $\iota(w)=k m w^{\prime}$. Then $\iota(w)$ satisfies the conditions of the lemma.

Assume that $\iota(w) \in N_{K}(\mathfrak{h})$ and $\iota(w)^{\prime} \in N_{K}(\mathfrak{h})$ satisfy these conditions. Put $w_{1}=\iota(w)^{-1} \iota(w)^{\prime} \in N_{K}(\mathfrak{h})$. Then $\left.\operatorname{Ad}\left(w_{1}\right)\right|_{\mathfrak{a}_{0}}=$ id, so $w_{1} \in N_{K}\left(\mathfrak{a}_{0}\right)=M_{0}$. Hence $w_{1}$ gives an element of the Weyl group of $M_{0}$. Consequently, $\operatorname{Ad}\left(w_{1}\right)\left(\Delta_{M_{0}}^{+}\right)=\Delta_{M_{0}}^{+}$. Hence $w_{1}$ centralizes $\mathfrak{h}$. Therefore, $\iota(w) \in \iota(w)^{\prime} Z_{K}(\mathfrak{h})$.

The correspondence $w \mapsto \iota(w)$ gives a map $\iota: W \rightarrow N_{K}(\mathfrak{h}) / Z_{K}(\mathfrak{h})$. By the characterization of $\iota(w)$, this map is injective. Since the group $N_{K}(\mathfrak{h}) / Z_{K}(\mathfrak{h})$ is a subgroup of $\widetilde{W}$, we can regard $W$ as a subgroup of $\widetilde{W}$. Hence we can define the twisting functor $T_{\iota(w), \psi}$ for $w \in W$ and the character $\psi$ of $\operatorname{Ad}(w) \overline{\mathfrak{n}_{0}} \cap \mathfrak{n}_{0}$. For simplicity, we write $w$ instead of $\iota(w)$. (We regard $W$ as a subgroup of $\widetilde{W}$ via $\iota$.)

Lemma 5.4. Let $e$ be a nilpotent element of $\mathfrak{g}, X \in \mathfrak{g}$ and $k \in \mathbb{Z}_{\geq 0}$. For $c \in \mathbb{C}$ we have the following equation in $U(\mathfrak{g})_{e-c}$ :

$$
X(e-c)^{-(k+1)}=\sum_{n=0}^{\infty}\left(\begin{array}{c}
n+k \\
k
\end{array}\right)(e-c)^{-(n+k+1)} \operatorname{ad}(e)^{n}(X) .
$$

Proof. We prove the lemma by induction on $k$. If $k=0$, the statement is wellknown. Assume that $k>0$. Then

$$
\begin{aligned}
X(e-c)^{-(k+1)} & =\sum_{k_{0}=0}^{\infty}(e-c)^{-\left(k_{0}+1\right)} \operatorname{ad}(e)^{k_{0}}(X)(e-c)^{-k} \\
& =\sum_{k_{0}=0}^{\infty} \sum_{k_{1}=0}^{\infty}\left(\begin{array}{c}
k_{1}+k-1 \\
k-1
\end{array}\right)(e-c)^{-\left(k_{0}+k_{1}+k+1\right)} \operatorname{ad}(e)^{k_{0}+k_{1}}(X) \\
& =\sum_{n=0}^{\infty} \sum_{l^{\prime}=0}^{n}\left(\begin{array}{c}
l^{\prime}+k-1 \\
k-1
\end{array}\right)(e-c)^{-(n+k+1)} \operatorname{ad}(e)^{n}(X) \\
& =\sum_{n=0}^{\infty}\left(\begin{array}{c}
n+k \\
k
\end{array}\right)(e-c)^{-(n+k+1)} \operatorname{ad}(e)^{n}(X) .
\end{aligned}
$$


$\S 6$. The module $I_{i} / I_{i-1}$

Put $J_{i}=U(\mathfrak{g}) \otimes_{U(\mathfrak{p})} J_{w_{i}^{-1} \eta}^{\prime}\left(\sigma \otimes e^{\lambda+\rho}\right)$, where $\mathfrak{n}$ acts on $J_{w_{i}^{-1} \eta}^{\prime}\left(\sigma \otimes e^{\lambda+\rho}\right)$ trivially. In this section, we prove the following theorem.

Theorem 6.1. Assume that $I_{i} / I_{i-1} \neq 0$. Then $I_{i} / I_{i-1} \simeq T_{w_{i}, \eta} J_{i}$.

Notice that $\mathfrak{u}_{0, w_{i}}=\operatorname{Ad}\left(w_{i}\right) \overline{\mathfrak{n}} \cap \mathfrak{n}_{0}$ since $w_{i}\left(\Delta_{M}^{+}\right) \subset \Delta^{+}$. In this section we fix $i \in\{1, \ldots, l\}$ and a basis $\left\{e_{1}, \ldots, e_{l}\right\}$ of $\mathfrak{u}_{0, w_{i}}$ such that each vector $e_{s}$ is a root vector and $\bigoplus_{s \leq t-1} \mathbb{C} e_{s}$ is an ideal of $\bigoplus_{s \leq t} \mathbb{C} e_{s}$. Let $\alpha_{s}$ be the restricted root corresponding to $e_{s}$. As in Section 3 , for $\mathbf{k}=\left(k_{1}, \ldots, k_{l}\right) \in \mathbb{Z}_{\geq 0}^{l}$ we denote $\operatorname{ad}\left(e_{l}\right)^{k_{l}} \cdots \operatorname{ad}\left(e_{1}\right)^{k_{1}}$ by $\operatorname{ad}(e)^{\mathbf{k}}$ and $\left(\left(-x_{1}\right)^{k_{1}} / k_{1} !\right) \cdots\left(\left(-x_{l}\right)^{k_{l}} / k_{l} !\right)$ by $f_{\mathbf{k}}$.

Lemma 6.2. We have

$$
I_{i}^{\prime}=\left\{\begin{array}{l|l}
\sum_{s=1}^{t} \delta_{i}\left(E_{s}, f_{s} \eta_{i}^{-1}, u_{s}^{\prime}\right) & \begin{array}{l}
E_{s} \in U(\mathfrak{g}), f_{s} \in \mathcal{P}\left(O_{i}\right), \\
u_{s}^{\prime} \in J_{w_{i}^{\prime} \eta}^{\prime}\left(\sigma \otimes e^{\lambda+\rho}\right)
\end{array}
\end{array}\right\} .
$$

Proof. By Lemma 3.3,

$$
E\left(\left(f \otimes u^{\prime}\right) \delta_{i}\right)=\sum_{\mathbf{k} \in \mathbb{Z}_{\geq 0}^{l}} \delta_{i}\left(\operatorname{ad}(e)^{\mathbf{k}} E, f f_{\mathbf{k}}, u^{\prime}\right)
$$

for $E \in U(\mathfrak{g}), f \in \mathcal{P}\left(O_{i}\right) \eta_{i}^{-1}$ and $u^{\prime} \in \sigma^{\prime}$. Hence, the left hand side in the statement is a subset of the right hand side. Define $f_{\mathbf{k}}^{\prime} \in \mathcal{P}\left(O_{i}\right)$ by $f_{\mathbf{k}}^{\prime}=\left(x_{1}^{k_{1}} / k_{1} !\right) \cdots\left(x_{l}^{k_{l}} / k_{l} !\right)$. By a similar calculation to the proof of Lemma 3.3, we have

$$
\delta_{i}\left(E, f, u^{\prime}\right)=\sum_{\mathbf{k} \in \mathbb{Z}_{\geq 0}^{l}}\left(\operatorname{ad}(e)^{\mathbf{k}} E\right)\left(\left(\left(f f_{\mathbf{k}}^{\prime}\right) \otimes u^{\prime}\right) \delta_{i}\right) .
$$

This implies that the right hand side is contained in the left hand side.

By the definition of the twisting functor and the Poincaré-Birkhoff-Witt theorem, we have the lemma below. For $\mathbf{k}=\left(k_{1}, \ldots, k_{l}\right) \in \mathbb{Z}^{l}$ put $(e-\eta(e))^{\mathbf{k}}=\left(e_{1}-\right.$ $\left.\eta\left(e_{1}\right)\right)^{k_{1}} \cdots\left(e_{l}-\eta\left(e_{l}\right)\right)^{k_{l}} \in S_{w_{i}, \eta}$. Set $\mathbf{1}=(1, \ldots, 1) \in \mathbb{Z}^{l}$. By multiplication from the right, the subspace $\sum_{\mathbf{k} \in \mathbb{Z}_{\geq 0}^{l}} \mathbb{C}(e-\eta(e))^{-(\mathbf{k}+\mathbf{1})} \subset S_{w_{i}, \eta}$ is a $U\left(\operatorname{Ad}\left(w_{i}\right) \overline{\mathfrak{n}} \cap \mathfrak{n}_{0}\right)$ submodule.

Lemma 6.3. Let $V$ be a p-module. Then

$$
\begin{aligned}
& \left(\sum_{\mathbf{k} \in \mathbb{Z}_{\geq 0}^{l}} \mathbb{C}(e-\eta(e))^{-(\mathbf{k}+\mathbf{1})}\right) \otimes U\left(\operatorname{Ad}\left(w_{i}\right) \overline{\mathfrak{n}} \cap \overline{\mathfrak{n}_{0}}\right) \otimes w_{i} V \\
& \quad \simeq\left(\sum_{\mathbf{k} \in \mathbb{Z}_{\geq 0}^{l}} \mathbb{C}(e-\eta(e))^{-(\mathbf{k}+\mathbf{1})}\right) \otimes_{U\left(\operatorname{Ad}\left(w_{i}\right) \overline{\mathfrak{n}} \cap \mathfrak{n}_{0}\right)} U(\mathfrak{g}) \otimes_{U\left(\operatorname{Ad}\left(w_{i}\right) \mathfrak{p}\right)} w_{i} V \\
& \quad \simeq T_{w_{i}, \eta}\left(U(\mathfrak{g}) \otimes_{U(\mathfrak{p})} V\right)
\end{aligned}
$$


The second isomorphism is given by $E \otimes F \otimes v \mapsto E F \otimes(1 \otimes v)$. (Notice that $\left.E F \in S_{w_{i}, \eta} \cdot\right)$

Proof of Theorem 6.1. By Lemmas 6.2 and 3.2, we have an isomorphism of vector spaces

$$
I_{i}^{\prime} \simeq \mathcal{P}\left(O_{i}\right) \otimes_{U\left(\operatorname{Ad}\left(w_{i}\right) \overline{\mathfrak{n}} \cap \mathfrak{n}_{0}\right)} U(\mathfrak{g}) \otimes_{U\left(\operatorname{Ad}\left(w_{i}\right) \mathfrak{p}\right)} w_{i} J_{w_{i}^{-1} \eta}^{\prime}\left(\sigma \otimes e^{\lambda+\rho}\right)
$$

given by $\delta_{i}\left(E, f, u^{\prime}\right) \mapsto f \otimes E \otimes u^{\prime}$.

Notice that $\mathfrak{u}_{0, w_{i}}=\operatorname{Ad}\left(w_{i}\right) \overline{\mathfrak{n}} \cap \mathfrak{n}_{0}$ since $w_{i} \in W(M)$. By Lemma 6.3,

$$
\begin{aligned}
T_{w_{i}, \eta}\left(J_{i}\right) \simeq & \left(\sum_{\mathbf{k} \in \mathbb{Z}_{\geq 0}^{l}} \mathbb{C}(e-\eta(e))^{-(\mathbf{k}+\mathbf{1})}\right) \otimes_{U\left(\operatorname{Ad}\left(w_{i}\right) \overline{\mathfrak{n}} \cap \mathfrak{n}_{0}\right)} U(\mathfrak{g}) \\
& \otimes_{U\left(\operatorname{Ad}\left(w_{i}\right) \mathfrak{p}\right)} w_{i} J_{w_{i}^{-1} \eta}^{\prime}\left(\sigma \otimes e^{\lambda+\rho}\right) .
\end{aligned}
$$

Here $\sum_{\mathbf{k} \in \mathbb{Z}_{\geq 0}^{l}} \mathbb{C}(e-\eta(e))^{-(\mathbf{k}+\mathbf{1})}$ is an $\operatorname{Ad}\left(w_{i}\right) \overline{\mathfrak{n}} \cap \mathfrak{n}_{0}$-stable subspace of $S_{w_{i}, \eta}$. Hence, we can define a $\mathbb{C}$-vector space isomorphism $\Phi: T_{w_{i}, \eta}\left(J_{i}\right) \rightarrow I_{i}^{\prime}$ by

$$
\Phi\left((e-\eta(e))^{-(\mathbf{k}+\mathbf{1})} \otimes E \otimes u^{\prime}\right)=\delta_{i}\left(E, f_{\mathbf{k}} \eta_{i}^{-1}, u^{\prime}\right) .
$$

We now prove that $\Phi$ is a $\mathfrak{g}$-homomorphism. Fix $X \in \mathfrak{g}$. We will prove that

$$
\Phi\left(X\left((e-\eta(e))^{-(\mathbf{k}+\mathbf{1})} \otimes E \otimes u^{\prime}\right)\right)=X \Phi\left((e-\eta(e))^{-(\mathbf{k}+\mathbf{1})} \otimes E \otimes u^{\prime}\right) .
$$

By Lemma 5.4,

$$
\begin{aligned}
& X\left((e-\eta(e))^{-(\mathbf{k}+\mathbf{1})} \otimes E \otimes u^{\prime}\right) \\
& \quad=\sum_{p_{s} \geq 0}\left(\begin{array}{c}
p_{1}+k_{1} \\
k_{1}
\end{array}\right) \cdots\left(\begin{array}{c}
p_{l}+k_{l} \\
k_{l}
\end{array}\right)(e-\eta(e))^{-(\mathbf{k}+\mathbf{p}+\mathbf{1})} \otimes\left(\operatorname{ad}(e)^{\mathbf{p}} X\right) E \otimes u^{\prime} .
\end{aligned}
$$

where $\mathbf{p}=\left(p_{1}, \ldots, p_{l}\right)$. Hence,

$$
\begin{aligned}
\Phi(X((e- & \left.\left.\eta(e))^{-(\mathbf{k}+\mathbf{1})} \otimes E \otimes u^{\prime}\right)\right) \\
& =\sum_{p_{s} \geq 0} \delta_{i}\left(\left(\operatorname{ad}(e)^{\mathbf{p}} X\right) E,\left(\frac{\left(-x_{1}\right)^{k_{1}+p_{1}}}{k_{1} ! p_{1} !} \cdots \frac{\left(-x_{l}\right)^{k_{l}+p_{l}}}{k_{l} ! p_{l} !}\right) \eta_{i}^{-1}, u^{\prime}\right) .
\end{aligned}
$$

By Lemma 3.3,

$$
\begin{aligned}
X \Phi\left((e-\eta(e))^{-(\mathbf{k}+1)} \otimes E \otimes u^{\prime}\right) & =X \delta_{i}\left(E, f_{\mathbf{k}} \eta_{i}^{-1}, u^{\prime}\right) \\
& =\sum_{\mathbf{p} \in \mathbb{Z}_{\geq 0}^{l}} \delta_{i}\left(\left(\operatorname{ad}(e)^{\mathbf{p}} X\right) E, f_{\mathbf{k}} f_{\mathbf{p}} \eta_{i}^{-1}, u^{\prime}\right) .
\end{aligned}
$$

Hence the conclusion follows. 


\section{$\S 7$. The module $J_{\eta}^{*}(I(\sigma, \lambda))$}

Now we investigate the module $J_{\eta}^{*}(I(\sigma, \lambda))$. For a finite-length moderate growth Fréchet representation $V$ of $G$, define a $\mathfrak{g}$-module $J(V)$ by

$$
J(V)=\left(\lim _{k \rightarrow \infty}\left(V_{K \text {-finite }} / \mathfrak{n}_{0}^{k} V_{K \text {-finite }}\right)\right)_{\mathfrak{a} \text {-finite }}
$$

This is also called the Jacquet module of $V$ [Cas80]. Define $\mathcal{O}_{P_{0}}^{\prime}$ to be the full subcategory of finitely generated $\mathfrak{g}$-modules $V$ satisfying the following conditions:

(1) The action of $\mathfrak{p}_{0}$ is locally finite. (In particular, the action of $\mathfrak{n}_{0}$ is locally nilpotent.)

(2) The module $V$ is $Z(\mathfrak{g})$-finite.

(3) The group $M_{0}$ acts on $V$ and its differential coincides with the action of $\mathfrak{m}_{0} \subset \mathfrak{g}$.

(4) For $\nu \in \mathfrak{a}_{0}^{*}$ let $V_{\nu}$ be the generalized $\mathfrak{a}_{0}$-weight space with weight $\nu$. Then $V=\bigoplus_{\nu \in \mathfrak{a}_{0}^{*}} V_{\nu}$ and $\operatorname{dim} V_{\nu}<\infty$.

We define $\mathcal{O}_{\overline{P_{0}}}^{\prime}$ similarly. We write $\mathcal{O}_{P_{0}, G}^{\prime}$ to emphasize the group $G$. Then for a finite-length Fréchet representation $V$ of $G$ we have $J(V) \in \mathcal{O}_{\overline{P_{0}}}^{\prime}$ and $J^{*}(V) \in \mathcal{O}_{P_{0}}^{\prime}$. For a $U(\mathfrak{g})$-module $V$, put $D^{\prime}(V)=\left(V^{*}\right)_{\mathfrak{h} \text {-finite }}$ and $C(V)=\left(D^{\prime}(V)\right)^{*}$. The character $\eta: \mathfrak{n}_{0} \rightarrow \mathbb{C}$ defines an algebra homomorphism $U\left(\mathfrak{n}_{0}\right) \rightarrow \mathbb{C}$ by the universality of the universal enveloping algebra. Let Ker $\eta$ be the kernel of this algebra homomorphism and put $\Gamma_{\eta}(V)=\left\{v \in V \mid(\operatorname{Ker} \eta)^{k} v=0\right.$ for some $\left.k\right\}$. Then $J_{\eta}^{*}(V)=\Gamma_{\eta}\left(\left(V_{K \text {-finite }}\right)^{*}\right)$ by Remark 2.2. We will prove the following proposition.

Proposition 7.1. Let $V$ be a finite-length moderate growth Fréchet representation of $G$. Then $J_{\eta}^{*}(V) \simeq \Gamma_{\eta}\left(J(V)^{*}\right) \simeq \Gamma_{\eta}\left(C\left(J^{*}(V)\right)\right)$.

From this proposition, Theorem 6.1 and the automatic continuity theorem [Wal83, Theorem 4.8], we get the structure of $J_{\eta}^{*}(I(\sigma, \lambda))$.

Proposition 7.1 was proved by Matumoto [Mat90, Theorem 4.9.2] when supp $\eta$ $=\Pi$. We deduce the general case from his theorem. To do this, we need some lemmas. We use the following well-known properties (see Wallach's book [Wal88]):

Proposition 7.2. Let $V$ be a finite-length moderate growth Fréchet representation of $G$.

(1) $D^{\prime}\left(J^{*}(V)\right) \simeq J(V)$

(2) $V / \mathfrak{n}_{0}^{k} V \simeq J(V) / \mathfrak{n}_{0}^{k} J(V)$.

(3) The functor $\Gamma_{\eta} \circ C$ from $\mathcal{O}_{P_{0}}^{\prime}$ or $\mathcal{O}_{\overline{P_{0}}}^{\prime}$ to the category of $\mathfrak{g}$-modules is exact.

(4) $D^{\prime}\left(\mathcal{O}_{P_{0}}^{\prime}\right) \subset \mathcal{O}_{\overline{P_{0}}}^{\prime}$ and $D^{\prime}\left(\mathcal{O}_{\overline{P_{0}}}^{\prime}\right) \subset \mathcal{O}_{P_{0}}^{\prime}$. If $V \in \mathcal{O}_{P_{0}}^{\prime}$ or $V \in \mathcal{O}_{\overline{P_{0}}}^{\prime}$, then $D^{\prime} D^{\prime} V \simeq V$. 
Lemma 7.3. Let $\mathfrak{c}$ be a nilpotent Lie algebra and $\psi$ its character. Denote the corresponding $\mathbb{C}$-algebra homomorphism $U(\mathfrak{c}) \rightarrow \mathbb{C}$ again by $\psi$, and its kernel by Ker $\psi$. Let $V$ be a $\mathfrak{c}$-module and $\mathfrak{c}_{1}, \mathfrak{c}_{2}$ subalgebras such that $\mathfrak{c}=\mathfrak{c}_{1} \oplus \mathfrak{c}_{2}$ and $\mathfrak{c}_{2}$ is an ideal of $\mathfrak{c}$. Set $\psi_{i}=\left.\psi\right|_{U\left(\mathfrak{c}_{i}\right)}$. Then

$$
\bigcup_{k}\left\{v \in V \mid(\operatorname{Ker} \psi)^{k} v=0\right\}=\bigcup_{k, l}\left\{v \in V \mid\left(\operatorname{Ker} \psi_{1}\right)^{k} v=0,\left(\operatorname{Ker} \psi_{2}\right)^{l} v=0\right\} .
$$

Proof. Replacing $V$ with $V \otimes(-\psi)$, we may assume $\psi$ is trivial. By the same proof as in Remark 2.2, if $\mathfrak{c}_{1}^{k} v_{0}=0, \mathfrak{c}_{2}^{l} v_{0}=0$, then there exists $k^{\prime}$ such that $\mathfrak{c}^{k^{\prime}} v_{0}=0$. Apply this to $v_{0}=1 \in V_{0}=U(\mathfrak{c}) /\left(U(\mathfrak{c}) \mathfrak{c}_{1}^{k}+U(\mathfrak{c}) \mathfrak{c}_{2}^{l}\right)$. Then there exists $k^{\prime}$ such that $\mathfrak{c}^{k^{\prime}} v_{0}=0$.

Take $v$ such that $\mathfrak{c}_{1}^{k} v=0, \mathfrak{c}_{2}^{l} v=0$. Then there exists a homomorphism $V_{0} \rightarrow V$ such that $v_{0} \mapsto v$. Hence $\mathfrak{c}^{k^{\prime}} v=0$. Therefore,

$$
\left\{v \in V \mid \mathfrak{c}_{1}^{k} v=0, \mathfrak{c}_{2}^{l} v=0\right\} \subset\left\{v \in V \mid \mathfrak{c}^{k^{\prime}} v=0\right\}
$$

On the other hand,

$$
\left\{v \in V \mid \mathfrak{c}^{k} v=0\right\} \subset\left\{v \in V \mid \mathfrak{c}_{1}^{k} v=0, \mathfrak{c}_{2}^{k} v=0\right\} .
$$

This implies the lemma.

From the above lemma, we get the lemma below. Recall that $\mathfrak{p}_{\eta}=\mathfrak{m}_{\eta} \oplus \mathfrak{a}_{\eta} \oplus \mathfrak{n}_{\eta}$ is the complexification of the Lie algebra of the parabolic subgroup corresponding to $\operatorname{supp} \eta($ Section 4$)$.

Lemma 7.4. Denote the $\mathbb{C}$-algebra homomorphism $U\left(\mathfrak{n}_{0}\right) \rightarrow \mathbb{C}$ corresponding to $\eta$ again by $\eta$. Put $\eta_{0}=\left.\eta\right|_{U\left(\mathfrak{m}_{\eta} \cap \mathfrak{n}_{0}\right)}$. Then for any $\mathfrak{g}$-module $V$, we have

$$
\Gamma_{\eta}(V)=\bigcup_{k, l}\left\{v \in V \mid \mathfrak{n}_{\eta}^{l} v=0,\left(\operatorname{Ker} \eta_{0}\right)^{k} v=0\right\} .
$$

Proof of Proposition 7.1. The second isomorphism follows from the definition of $C, D^{\prime}$ and Proposition 7.2(1).

We will prove $J_{\eta}^{*}(V) \simeq \Gamma_{\eta}\left(J(V)^{*}\right)$. If $\operatorname{supp} \eta=\Pi$, this was proved by Matumoto [Mat90, Theorem 4.9.2].

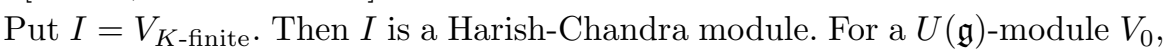

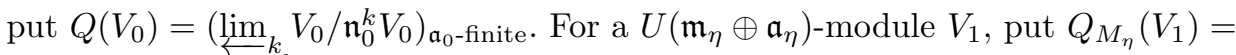

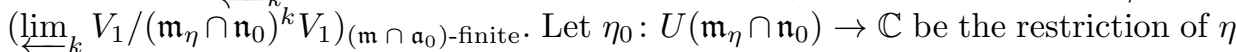
to $U\left(\mathfrak{m}_{\eta} \cap \mathfrak{n}_{0}\right)$. Since $I / \mathfrak{n}_{\eta}^{l} I$ is a Harish-Chandra module of $\mathfrak{m}_{\eta} \oplus \mathfrak{a}_{\eta}$, by the result of Matumoto we have $\Gamma_{\eta_{0}}\left(\left(I / \mathfrak{n}_{\eta}^{l} I\right)^{*}\right)=\Gamma_{\eta_{0}}\left(Q_{M_{\eta}}\left(I / \mathfrak{n}_{\eta}^{l} I\right)^{*}\right)$. Therefore,

$$
\left\{v \in\left(I / \mathfrak{n}_{\eta}^{l} I\right)^{*} \mid\left(\operatorname{Ker} \eta_{0}\right)^{k} v=0\right\}=\left\{v \in Q_{M_{\eta}}\left(I / \mathfrak{n}_{\eta}^{l} I\right)^{*} \mid\left(\operatorname{Ker} \eta_{0}\right)^{k} v=0\right\}
$$

for all $k \in \mathbb{Z}_{\geq 0}$. 
We will prove $Q_{M_{\eta}}\left(I / \mathfrak{n}_{\eta}^{l} I\right) \simeq Q(I) / \mathfrak{n}_{\eta}^{l} Q(I)$. It is sufficient to show that $D^{\prime}\left(Q_{M_{\eta}}\left(I / \mathfrak{n}_{\eta}^{l} I\right)\right) \simeq D^{\prime}\left(Q(I) / \mathfrak{n}_{\eta}^{l} Q(I)\right)$. By Proposition 7.2(1),

$$
\begin{aligned}
D^{\prime}\left(Q_{M_{\eta}}\left(I / \mathfrak{n}_{\eta}^{l} I\right)\right) & \simeq\left\{v \in\left(I / \mathfrak{n}_{\eta}^{l} I\right)^{*} \mid\left(\mathfrak{m}_{\eta} \cap \mathfrak{n}_{0}\right)^{k} v=0 \text { for some } k\right\} \\
& \simeq\left\{v \in I^{*} \mid \mathfrak{n}_{\eta}^{l} v=0,\left(\mathfrak{m}_{\eta} \cap \mathfrak{n}_{0}\right)^{k} v=0 \text { for some } k\right\} \\
& =\left\{v \in I^{*} \mid \mathfrak{n}_{\eta}^{l} v=0, \mathfrak{n}_{0}^{k} v=0 \text { for some } k\right\}
\end{aligned}
$$

Using Proposition 7.2(1) again, we obtain

$$
\left\{v \in I^{*} \mid \mathfrak{n}_{0}^{k} v=0 \text { for some } k\right\} \simeq D^{\prime}(Q(I)) .
$$

Hence

$$
D^{\prime}\left(Q_{M_{\eta}}\left(I / \mathfrak{n}_{\eta}^{l} I\right)\right) \simeq\left\{v \in D^{\prime}(Q(I)) \mid \mathfrak{n}_{\eta}^{l} v=0\right\}
$$

By its definition, $D^{\prime}$ is left exact. Hence we have an exact sequence

$$
0 \rightarrow D^{\prime}\left(Q(I) / \mathfrak{n}_{\eta}^{l} Q(I)\right) \rightarrow D^{\prime}(Q(I)) \rightarrow D^{\prime}\left(\mathfrak{n}_{\eta}^{l} Q(I)\right)
$$

Therefore, $\left\{v \in D^{\prime}(Q(I)) \mid \mathfrak{n}_{\eta}^{l} v=0\right\} \simeq D^{\prime}\left(Q(I) / \mathfrak{n}_{\eta}^{l} Q(I)\right)$. Hence $Q_{M_{\eta}}\left(I / \mathfrak{n}_{\eta}^{l} I\right) \simeq$ $Q(I) / \mathfrak{n}_{\eta}^{l} Q(I)$. This implies

$$
\left\{v \in\left(I / \mathfrak{n}_{\eta}^{l} I\right)^{*} \mid\left(\operatorname{Ker} \eta_{0}\right)^{k} v=0\right\} \simeq\left\{v \in\left(Q(I) / \mathfrak{n}_{\eta}^{l} Q(I)\right)^{*} \mid\left(\operatorname{Ker} \eta_{0}\right)^{k} v=0\right\} .
$$

Hence

$$
\left\{v \in I^{*} \mid \mathfrak{n}_{\eta}^{l} v=0,\left(\operatorname{Ker} \eta_{0}\right)^{k} v=0\right\} \simeq\left\{v \in Q(I)^{*} \mid \mathfrak{n}_{\eta}^{l} v=0,\left(\operatorname{Ker} \eta_{0}\right)^{k} v=0\right\}
$$

Therefore, by the previous lemma, we have

$$
\Gamma_{\eta}\left(I^{*}\right) \simeq \Gamma_{\eta}\left(Q(I)^{*}\right)
$$

By the definition and Remark 2.2, $Q(I)=J(V)$ and $\Gamma_{\eta}\left(I^{*}\right)=J_{\eta}^{*}(I)$.

Combining Theorem 6.1, Proposition 7.1 and the automatic continuity theorem [Wal83, Theorem 4.8], we have the following theorem. Let $I_{i}$ be the Bruhat filtration of $J^{\prime}(I(\sigma, \lambda)) \simeq J^{*}(I(\sigma, \lambda))$. Put $\widetilde{I}_{i}=\Gamma_{\eta}\left(C\left(I_{i}\right)\right) \subset \Gamma_{\eta}\left(C\left(J^{*}(I(\sigma, \lambda))\right)\right) \simeq$ $J_{\eta}^{*}(I(\sigma, \lambda))$.

Theorem 7.5. The filtration $0=\widetilde{I_{1}} \subset \cdots \subset \widetilde{I_{r}}=J_{\eta}^{*}(I(\sigma, \lambda))$ satisfies $\widetilde{I_{i}} / \widetilde{I_{i-1}} \simeq$ $\Gamma_{\eta}\left(C\left(T_{w_{i}}\left(U(\mathfrak{g}) \otimes_{U(\mathfrak{p})} J^{*}\left(\sigma \otimes e^{\lambda+\rho}\right)\right)\right)\right)$.

Proof. This follows from Theorem 6.1 and Propositions 7.2 and 7.1. 


\section{$\S 8$. Whittaker vectors}

We now study the space of Whittaker vectors of $I(\sigma, \lambda)^{\prime}$ and $\left(I(\sigma, \lambda)_{K \text {-finite }}\right)^{*}$ (Definition 3.9) using the Bruhat filtration.

First, we consider $\mathrm{Wh}_{\eta}\left(I(\sigma, \lambda)^{\prime}\right)$. To calculate its dimension, we calculate $\operatorname{dim} \mathrm{Wh}_{\eta}\left(I_{i} / I_{i-1}\right)$. The idea is to use the Harish-Chandra isomorphism. To explain the idea, recall a proof of the following fact: the Verma module has a unique highest weight if its infinitesimal character is generic. (Here, for a $\mathfrak{g}$-module $V$, we call $\widetilde{\lambda} \in \mathfrak{h}^{*}$ a highest weight of $V$ if $\widetilde{\lambda}$ is the weight of a vector in $V$ killed by the nilpotent radical of the Borel subalgebra.) The proof is the following. Let $\widetilde{\lambda}$ be the infinitesimal character of the Verma module and assume that the set of weights of the Verma module is $\widetilde{\lambda}+\mathbb{Z}_{\leq 0} \Delta$. Then by the Harish-Chandra isomorphism, each highest weight of the Verma module has the form $\widetilde{w}(\widetilde{\lambda}+\widetilde{\rho})-\widetilde{\rho}$. Therefore, $\widetilde{w} \widetilde{\lambda}-\widetilde{\lambda} \in \mathbb{Z} \Delta$. Since $\widetilde{\lambda}$ is generic, $\widetilde{w}=1$. We use an analogous proof. To do it, we decompose the Harish-Chandra homomorphism, using the following lemma.

Lemma 8.1. If $I_{i} / I_{i-1} \neq 0$, then $\mathfrak{l}_{\eta} \cap \operatorname{Ad}\left(w_{i}\right) \overline{\mathfrak{n}} \subset \mathfrak{n}_{0}$.

Proof. By Lemma 3.6, the restriction of $\eta$ to $\operatorname{Ad}\left(w_{i}\right) \mathfrak{n} \cap \mathfrak{n}_{0}$ is trivial. This is equivalent to supp $\eta \cap w_{i}\left(\Sigma^{+} \backslash \Sigma_{M}^{+}\right) \cap \Sigma^{+}=\emptyset$. Thus, $\left(-\operatorname{supp} \eta \cap \Sigma^{-}\right) \cap w_{i}\left(\Sigma^{-} \backslash \Sigma_{M}^{-}\right)=\emptyset$, so $\left(\mathfrak{l}_{\eta} \cap \overline{\mathfrak{n}_{0}}\right) \cap \operatorname{Ad}\left(w_{i}\right) \overline{\mathfrak{n}}=0$.

For $i$ such that $I_{i} / I_{i-1} \neq 0$, we define $\gamma_{1}$ to $\gamma_{4}$ to be the first projections with respect to the corresponding decompositions below:

$$
\begin{aligned}
& \gamma_{1}: U(\mathfrak{g})=U\left(\mathfrak{l}_{\eta}\right) \oplus\left(\overline{\mathfrak{n}_{\eta}} U(\mathfrak{g})+U(\mathfrak{g}) \mathfrak{n}_{\eta}\right) \rightarrow U\left(\mathfrak{l}_{\eta}\right), \\
& \gamma_{2}: U\left(\mathfrak{l}_{\eta}\right)=\left.U\left(\mathfrak{l}_{\eta} \cap \operatorname{Ad}\left(w_{i}\right) \mathfrak{p}\right) \oplus U\left(\mathfrak{l}_{\eta}\right) \operatorname{Ker} \eta\right|_{\mathfrak{l}_{\eta} \cap \operatorname{Ad}\left(w_{i}\right) \overline{\mathfrak{n}}} \rightarrow U\left(\mathfrak{l}_{\eta} \cap \operatorname{Ad}\left(w_{i}\right) \mathfrak{p}\right), \\
& \gamma_{3}: U\left(\mathfrak{l}_{\eta} \cap \operatorname{Ad}\left(w_{i}\right) \mathfrak{p}\right)=U\left(\mathfrak{l}_{\eta} \cap \operatorname{Ad}\left(w_{i}\right) \mathfrak{l}\right) \oplus\left(\mathfrak{l}_{\eta} \cap \operatorname{Ad}\left(w_{i}\right) \mathfrak{n}\right) U\left(\mathfrak{l}_{\eta} \cap \operatorname{Ad}\left(w_{i}\right) \mathfrak{p}\right) \\
& \rightarrow U\left(\mathfrak{l}_{\eta} \cap \operatorname{Ad}\left(w_{i}\right) \mathfrak{l}\right), \\
& \gamma_{4}: U\left(\mathfrak{l}_{\eta} \cap \operatorname{Ad}\left(w_{i}\right) \mathfrak{l}\right)=U(\mathfrak{h}) \oplus\left(\left(\overline{\mathfrak{u}_{0}} \cap \mathfrak{l}_{\eta} \cap \operatorname{Ad}\left(w_{i}\right) \mathfrak{l}\right) U\left(\mathfrak{l}_{\eta} \cap \operatorname{Ad}\left(w_{i}\right) \mathfrak{l}\right)\right. \\
& \left.\rightarrow U\left(\mathfrak{l}_{\eta} \cap \operatorname{Ad}\left(w_{i}\right) \mathfrak{l}\right)\left(\mathfrak{l}_{\eta} \cap \operatorname{Ad}\left(w_{i}\right) \mathfrak{l} \cap \mathfrak{u}_{0}\right)\right) \rightarrow U(\mathfrak{h}) .
\end{aligned}
$$

To define $\gamma_{2}$, we must check $\mathfrak{l}_{\eta} \cap \operatorname{Ad}\left(w_{i}\right) \overline{\mathfrak{n}} \subset \mathfrak{n}_{0}$. This follows from $I_{i} / I_{i-1} \neq 0$ and the previous lemma. Then the restriction of $\gamma_{4} \circ \gamma_{3} \circ \gamma_{2} \circ \gamma_{1}$ to $Z(\mathfrak{g})$ is the (non-shifted) Harish-Chandra homomorphism. If $x \in \mathrm{Wh}_{\eta}\left(I_{i} / I_{i-1}\right)$ then $E x=$ $\gamma_{2} \gamma_{1}(E) x$ for $E \in Z(\mathfrak{g})$.

Lemma 8.2. Let $V$ be a $U(\mathfrak{g})$-module with infinitesimal character $\widetilde{\lambda}$, and $\chi a$ character of $Z(\mathfrak{g})$ such that $z \in Z(\mathfrak{g})$ acts by $\chi(z)$ on $V$. Let $v \in V \backslash\{0\}$ and $\mu \in \mathfrak{a}^{*}$ be such that $\left(\gamma_{3} \gamma_{2} \gamma_{1}(z)-\chi(z)\right) v=0$ and $H v=\left(w_{i} \mu+\rho_{0}\right)(H) v$ for all $z \in Z(\mathfrak{g})$ and $H \in \operatorname{Ad}\left(w_{i}\right) \mathfrak{a}$. Then there exists $\widetilde{w} \in \widetilde{W}$ such that $\left.\widetilde{w} \widetilde{\lambda}\right|_{\mathfrak{a}}=\mu$. 
Proof. Put $Z=\gamma_{3} \gamma_{2} \gamma_{1}(Z(\mathfrak{g})) U\left(\operatorname{Ad}\left(w_{i}\right) \mathfrak{a}\right)$. By assumption, there exists a character $\chi_{0}$ of $Z$ such that $z v=\chi_{0}(z) v$ for all $z \in Z$. By a theorem of Harish-Chandra, $\left.\gamma_{4}\right|_{Z}$ is injective and finite. Hence there exists $\widetilde{\lambda_{1}} \in \mathfrak{h}^{*}$ such that $\widetilde{\lambda_{1}} \circ \gamma_{4}=\chi_{0}$ where we denote the algebra homomorphism $U(\mathfrak{h}) \rightarrow \mathbb{C}$ corresponding to $\widetilde{\lambda_{1}}$ again by $\widetilde{\lambda_{1}}$. Since $V$ has infinitesimal character $\widetilde{\lambda}$, we have $\widetilde{\lambda_{1}} \in \widetilde{W} \widetilde{\lambda}+\widetilde{\rho}$. Since $\gamma_{4}$ is trivial on $U\left(\operatorname{Ad}\left(w_{i}\right) \mathfrak{a}\right),\left.\widetilde{\lambda_{1}}\right|_{\operatorname{Ad}\left(w_{i}\right) \mathfrak{a}}=\left.\left(w_{i} \mu+\rho_{0}\right)\right|_{\operatorname{Ad}\left(w_{i}\right) \mathfrak{a}}$. The restriction of $\widetilde{\rho}$ to $\mathfrak{a}_{0}$ is $\rho_{0}$. Hence $\left.\widetilde{\rho}\right|_{\operatorname{Ad}\left(w_{i}\right) \mathfrak{a}}=\left.\rho_{0}\right|_{\operatorname{Ad}\left(w_{i}\right) \mathfrak{a}}$. Then for some $\widetilde{w} \in \widetilde{W}$ we have $\left.w_{i} \mu\right|_{\operatorname{Ad}\left(w_{i}\right) \mathfrak{a}}=\left.\widetilde{w} \widetilde{\lambda}\right|_{\operatorname{Ad}\left(w_{i}\right) \mathfrak{a}}$, proving the lemma.

Lemma 8.3. Let $X_{1}, \ldots, X_{n} \in \mathfrak{g}, f_{1} \in C^{\infty}\left(O_{i}\right), f_{2} \in C^{\infty}\left(U_{i}\right), u^{\prime} \in\left(\sigma \otimes e^{\lambda+\rho}\right)^{\prime}$. Assume that $R\left(\operatorname{Ad}\left(w_{i}\right)^{-1} X_{s}\right)\left(f_{2}\right)=0$ for all $s=1, \ldots, n$. Then

$$
\delta_{i}\left(X_{1} \cdots X_{n}, f_{1} f_{2}, u^{\prime}\right)=\delta_{i}\left(X_{1} \cdots X_{n}, f_{1}, u^{\prime}\right) f_{2} .
$$

Proof. Put $E=X_{1} \cdots X_{n}$. By assumption and Leibniz's rule, we have

$$
f_{2}\left(n w_{i}\right)\left(R\left(\operatorname{Ad}\left(w_{i}\right)^{-1} E\right) \varphi\right)\left(n w_{i}\right)=\left(R\left(\operatorname{Ad}\left(w_{i}\right)^{-1} E\right)\left(\varphi f_{2}\right)\right)\left(n w_{i}\right) .
$$

Hence, by definition, for $\varphi \in C_{c}^{\infty}\left(U_{i}, \mathcal{L}\right)$, we have

$$
\begin{aligned}
\left\langle\delta_{i}\left(E, f_{1} f_{2}, u^{\prime}\right), \varphi\right\rangle & =\int_{w_{i} \bar{N} w_{i}^{-1} \cap N_{0}} f_{1}\left(n w_{i}\right) f_{2}\left(n w_{i}\right)\left(u^{\prime}\left(R\left(\operatorname{Ad}\left(w_{i}\right)^{-1} E\right) \varphi\right)\left(n w_{i}\right)\right) d n \\
& =\int_{w_{i} \bar{N} w_{i}^{-1} \cap N_{0}} f_{1}\left(n w_{i}\right)\left(u^{\prime}\left(R\left(\operatorname{Ad}\left(w_{i}\right)^{-1} E\right)\left(\varphi f_{2}\right)\right)\left(n w_{i}\right)\right) d n \\
& =\left\langle\delta_{i}\left(E, f_{1}, u^{\prime}\right), f_{2} \varphi\right\rangle=\left\langle\delta_{i}\left(E, f_{1}, u^{\prime}\right) f_{2}, \varphi\right\rangle,
\end{aligned}
$$

and the lemma follows.

Recall that the $C^{\infty}$-function $\eta_{i}$ on $O_{i}$ is defined by $\eta_{i}\left(n w_{i} P / P\right)=\eta(n)$ for $n \in w_{i} \bar{N} w_{i}^{-1} \cap N_{0}$. For $\nu \in \mathfrak{a}^{*}$ put

$$
V(\nu)=\left\{\begin{array}{l|l}
\sum_{s} \delta_{i}\left(F_{s}, h_{s}, v_{s}^{\prime}\right) & \begin{array}{l}
F_{s} \in U\left(\operatorname{Ad}\left(w_{i}\right) \overline{\mathfrak{n}} \cap \overline{\mathfrak{n}_{0}}\right), h_{s} \in \mathcal{P}\left(O_{i}\right), \\
v_{s}^{\prime} \in J_{w_{i}^{-1} \eta}^{\prime}\left(\sigma \otimes e^{\lambda+\rho}\right), \\
\left.w_{i}^{-1}\left(\operatorname{wt} h_{s}+\operatorname{wt} F_{s}\right)\right)\left.\right|_{\mathfrak{a}}=\nu
\end{array}
\end{array}\right\} .
$$

Here, wt $h_{s}$ is the $\mathfrak{a}_{0}$-weight of $h_{s}$ with respect to $D_{i}$ (see page 430 ) and wt $F_{s}$ is the $\mathfrak{a}_{0}$-weight of $F_{s}$ with respect to the adjoint action. We have no weight in $I_{i} / I_{i-1}$. The spaces $V(\nu)$ play the role of weight spaces.

Remark 8.4. By Lemma 3.2(1), we have

$$
V(\nu)=\left\{\begin{array}{l|l}
\sum_{s} \delta_{i}\left(F_{s}, h_{s}, v_{s}^{\prime}\right) & \begin{array}{l}
F_{s} \in U\left(\operatorname{Ad}\left(w_{i}\right) \overline{\mathfrak{n}}\right), h_{s} \in \mathcal{P}\left(O_{i}\right), \\
v_{s}^{\prime} \in J_{w_{i}^{-1} \eta}^{\prime}\left(\sigma \otimes e^{\lambda+\rho}\right), \\
\left.\left(w_{i}^{-1}\left(\mathrm{wt} h_{s}+\mathrm{wt} F_{s}\right)\right)\right|_{\mathfrak{a}}=\nu
\end{array}
\end{array}\right\} .
$$


Lemma 8.5. Let $X \in U(\mathfrak{g})$ be an $\mathfrak{a}_{0}$-weight vector. Then

$$
X V(\nu) \subset V\left(\nu+\left.w_{i}^{-1} \operatorname{wt}(X)\right|_{\mathfrak{a}}\right) .
$$

Proof. We may assume $X \in \mathfrak{g}$. Let $\delta_{i}\left(E, f, u^{\prime}\right) \in V(\nu)$. By Lemma 3.3, we have

$$
X \delta_{i}\left(E, f, u^{\prime}\right)=\sum_{\mathbf{k} \in \mathbb{Z}_{\geq 0}^{l}} \delta_{i}\left(\left(\operatorname{ad}(e)^{\mathbf{k}} X\right) E, f f_{\mathbf{k}}, u^{\prime}\right) .
$$

Assume $\operatorname{ad}(e)^{\mathbf{k}} X \in \operatorname{Ad}\left(w_{i}\right) \mathfrak{p}$. Then

$$
\delta_{i}\left(\left(\operatorname{ad}(e)^{\mathbf{k}} X\right) E, f f_{\mathbf{k}}, u^{\prime}\right)=\delta_{i}\left(E, f f_{\mathbf{k}}, \operatorname{Ad}\left(w_{i}\right)^{-1}\left(\left(\operatorname{ad}(e)^{\mathbf{k}} X\right)\right) u^{\prime}\right) .
$$

If $\operatorname{ad}(e)^{\mathbf{k}}(X) \in \operatorname{Ad}\left(w_{i}\right) \mathfrak{n}$, then this is 0 . If $\operatorname{ad}(e)^{\mathbf{k}}(X) \in \operatorname{Ad}\left(w_{i}\right) \mathfrak{l}$, then we have $\left.w_{i}^{-1} \operatorname{wt}\left(\operatorname{ad}(e)^{\mathbf{k}} X\right)\right|_{\mathfrak{a}}=0$. Hence $\left.w_{i}^{-1} \operatorname{wt}(X)\right|_{\mathfrak{a}}=\left.w_{i}^{-1} \operatorname{wt}\left(f_{\mathbf{k}}\right)\right|_{\mathfrak{a}}$. Therefore, $\left.w_{i}^{-1}\left(\operatorname{wt}(E)+\operatorname{wt}\left(f f_{\mathbf{k}}\right)\right)\right|_{\mathfrak{a}}=\nu+\left.w_{i}^{-1} \operatorname{wt}(X)\right|_{\mathfrak{a}}$.

If $\operatorname{ad}(e)^{\mathbf{k}}(X) \in \operatorname{Ad}\left(w_{i}\right) \overline{\mathfrak{n}}$, then $\left(\operatorname{ad}(e)^{\mathbf{k}} X\right) E \in \operatorname{Ad}\left(w_{i}\right) \overline{\mathfrak{n}}$. We have

$$
w_{i}^{-1}\left(\operatorname{wt}\left(\operatorname{ad}(e)^{\mathbf{k}} X\right) E+\operatorname{wt} f f_{\mathbf{k}}\right)=w_{i}^{-1}(\operatorname{wt} E+\operatorname{wt} f+\operatorname{wt} X) .
$$

This implies the lemma.

Lemma 8.6. Define $\widetilde{\eta}_{i} \in C^{\infty}\left(U_{i}\right)$ by $\widetilde{\eta}_{i}\left(n n_{0} w_{i} P / P\right)=\eta_{i}(n)$ for $n \in w_{i} \bar{N} w_{i}^{-1} \cap N_{0}$ and $n_{0} \in w_{i} \bar{N} w_{i}^{-1} \cap \overline{N_{0}}$. Let $X \in U(\mathfrak{g})$. Assume that $X$ is an $\mathfrak{a}_{0}$-weight vector. For $\delta_{i}\left(E, f, u^{\prime}\right) \in V(\nu)$, we have

$$
X \delta_{i}\left(E, f \eta_{i}^{-1}, u^{\prime}\right)-\left(X \delta_{i}\left(E, f, u^{\prime}\right)\right) \widetilde{\eta}_{i}^{-1} \in \sum_{\nu^{\prime}>\nu} V\left(\nu^{\prime}+w_{i}^{-1} \text { wt }\left.X\right|_{\mathfrak{a}}\right) \widetilde{\eta}_{i}^{-1} .
$$

Here, wt $X$ is the $\mathfrak{a}_{0}$-weight of $X$ with respect to the adjoint action.

Proof. Fix a basis $\left\{e_{1}, \ldots, e_{l}\right\}$ of $\mathfrak{u}_{0, w_{i}}$ such that each $e_{s}$ is a root vector and $\bigoplus_{s \leq t-1} \mathbb{C} e_{s}$ is an ideal of $\bigoplus_{s \leq t} \mathbb{C} e_{s}$. Let $\alpha_{s}$ be the restricted root of $e_{s}$. As in Section 3 , for $\mathbf{k}=\left(k_{1}, \ldots, k_{l}\right) \in \mathbb{Z}_{\geq 0}^{l}$ we denote $\operatorname{ad}\left(e_{l}\right)^{k_{l}} \cdots \operatorname{ad}\left(e_{1}\right)^{k_{1}}$ by ad $(e)^{\mathbf{k}}$ and $\left(\left(-x_{1}\right)^{k_{1}} / k_{1} !\right) \cdots\left(\left(-x_{l}\right)^{k_{l}} / k_{l} !\right)$ by $f_{\mathbf{k}}$. By Lemma 3.3 ,

$$
X \delta_{i}\left(E, f \eta_{i}^{-1}, u^{\prime}\right)=\sum_{\mathbf{k} \in \mathbb{Z}_{\geq 0}^{l}} \delta_{i}\left(\left(\operatorname{ad}(e)^{\mathbf{k}} X\right) E, f f_{\mathbf{k}} \eta_{i}^{-1}, u^{\prime}\right) .
$$

Take $a_{\mathbf{k}}^{(p)} \in U\left(\operatorname{Ad}\left(w_{i}\right) \overline{\mathfrak{n}} \cap \mathfrak{n}_{0}\right), b_{\mathbf{k}}^{(p)} \in U\left(\operatorname{Ad}\left(w_{i}\right) \overline{\mathfrak{n}} \cap \overline{\mathfrak{n}_{0}}\right)$ and $c_{\mathbf{k}}^{(p)} \in U\left(\operatorname{Ad}\left(w_{i}\right) \mathfrak{p}\right)$ such that $\left(\operatorname{ad}(e)^{\mathbf{k}} X\right) E=\sum_{p} a_{\mathbf{k}}^{(p)} b_{\mathbf{k}}^{(p)} c_{\mathbf{k}}^{(p)}$ and $\operatorname{wt}\left(\left(\operatorname{ad}(e)^{\mathbf{k}} X\right) E\right)=\operatorname{wt} a_{\mathbf{k}}^{(p)}+\operatorname{wt} b_{\mathbf{k}}^{(p)}+$ $\operatorname{wt} c_{\mathbf{k}}^{(p)}$. Then

$$
\begin{aligned}
\delta_{i}\left(\left(\operatorname{ad}(e)^{\mathbf{k}} X\right) E, f f_{\mathbf{k}} \eta_{i}^{-1}, u^{\prime}\right) & =\sum_{p} \delta_{i}\left(a_{\mathbf{k}}^{(p)} b_{\mathbf{k}}^{(p)} c_{\mathbf{k}}^{(p)}, f f_{\mathbf{k}} \eta_{i}^{-1}, u^{\prime}\right) \\
& =\sum_{p} \delta_{i}\left(b_{\mathbf{k}}^{(p)}, R_{i}^{\prime}\left(\left(a_{\mathbf{k}}^{(p)}\right)^{\vee}\right)\left(f f_{\mathbf{k}} \eta_{i}^{-1}\right), \operatorname{Ad}\left(w_{i}\right)^{-1}\left(c_{\mathbf{k}}^{(p)}\right) u^{\prime}\right) .
\end{aligned}
$$


By the Leibniz rule, there is a finite subset $\mathcal{A}_{\mathbf{k}}^{(p)} \subset\left\{\left(a^{\prime}, a^{\prime \prime}\right) \in U\left(\operatorname{Ad}\left(w_{i}\right) \overline{\mathfrak{n}} \cap \mathfrak{n}_{0}\right)^{2} \mid\right.$ wt $a^{\prime}+$ wt $a^{\prime \prime}=$ wt $\left.a_{\mathbf{k}}^{(p)}, a^{\prime \prime} \notin \mathbb{C}\right\}$ such that

$$
\begin{aligned}
\delta_{i}\left(b_{\mathbf{k}}^{(p)}, R_{i}^{\prime}\left(\left(a_{\mathbf{k}}^{(p)}\right)^{\vee}\right)\left(f f_{\mathbf{k}} \eta_{i}^{-1}\right)-R_{i}^{\prime}\left(\left(a_{\mathbf{k}}^{(p)}\right)^{\vee}\right)\left(f f_{\mathbf{k}}\right) \eta_{i}^{-1}, \operatorname{Ad}\left(w_{i}\right)^{-1} c_{\mathbf{k}}^{(p)} u^{\prime}\right) \\
=\sum_{\left(a^{\prime}, a^{\prime \prime}\right) \in \mathcal{A}_{\mathbf{k}}^{(p)}} \delta_{i}\left(b_{\mathbf{k}}^{(p)}, R_{i}^{\prime}\left(a^{\prime}\right)\left(f f_{\mathbf{k}}\right) R_{i}^{\prime}\left(a^{\prime \prime}\right)\left(\eta_{i}^{-1}\right), \operatorname{Ad}\left(w_{i}\right)^{-1} c_{\mathbf{k}}^{(p)} u^{\prime}\right) \\
=\sum_{\left(a^{\prime}, a^{\prime \prime}\right) \in \mathcal{A}_{\mathbf{k}}^{(p)}}-\eta\left(a^{\prime \prime}\right) \delta_{i}\left(b_{\mathbf{k}}^{(p)}, R_{i}^{\prime}\left(a^{\prime}\right)\left(f f_{\mathbf{k}}\right) \eta_{i}^{-1}, \operatorname{Ad}\left(w_{i}\right)^{-1} c_{\mathbf{k}}^{(p)} u^{\prime}\right) .
\end{aligned}
$$

By the definition of $\tilde{\eta}_{i}$, we have $R\left(\operatorname{Ad}\left(w_{i}\right)^{-1} X^{\prime}\right) \widetilde{\eta}_{i}=0$ for $X^{\prime} \in \operatorname{Ad}\left(w_{i}\right) \overline{\mathfrak{n}} \cap \overline{\mathfrak{n}_{0}}$. Hence by Lemma 8.3,

$$
\delta_{i}\left(b_{\mathbf{k}}^{(p)}, f^{\prime} \eta_{i}^{-1}, \operatorname{Ad}\left(w_{i}\right)^{-1}\left(c_{\mathbf{k}}^{(p)}\right) u^{\prime}\right)=\delta_{i}\left(b_{\mathbf{k}}^{(p)}, f^{\prime}, \operatorname{Ad}\left(w_{i}\right)^{-1}\left(c_{\mathbf{k}}^{(p)}\right) u^{\prime}\right) \widetilde{\eta}_{i}^{-1}
$$

for all $f^{\prime} \in \mathcal{P}\left(O_{i}\right)$. Thus

$$
\begin{aligned}
\delta_{i}\left(b_{\mathbf{k}}^{(p)}, R_{i}^{\prime}\left(\left(a_{\mathbf{k}}^{(p)}\right)^{\vee}\right)\left(f f_{\mathbf{k}} \eta_{i}^{-1}\right), \operatorname{Ad}\left(w_{i}\right)^{-1} c_{\mathbf{k}}^{(p)} u^{\prime}\right) & \\
& -\delta_{i}\left(b_{\mathbf{k}}^{(p)}, R_{i}^{\prime}\left(\left(a_{\mathbf{k}}^{(p)}\right)^{\vee}\right)\left(f f_{\mathbf{k}}\right), \operatorname{Ad}\left(w_{i}\right)^{-1} c_{\mathbf{k}}^{(p)} u^{\prime}\right) \widetilde{\eta}_{i}^{-1} \\
= & \sum_{\left(a^{\prime}, a^{\prime \prime}\right) \in \mathcal{A}_{\mathbf{k}}^{(p)}}-\eta\left(a^{\prime \prime}\right) \delta_{i}\left(b_{\mathbf{k}}^{(p)}, R_{i}^{\prime}\left(a^{\prime}\right)\left(f f_{\mathbf{k}}\right), \operatorname{Ad}\left(w_{i}\right)^{-1} c_{\mathbf{k}}^{(p)} u^{\prime}\right) \widetilde{\eta}_{i}^{-1} .
\end{aligned}
$$

By the Poincaré-Birkhoff-Witt theorem, we have a decomposition $U\left(\operatorname{Ad}\left(w_{i}\right) \mathfrak{p}\right)$ $=U\left(\operatorname{Ad}\left(w_{i}\right) \mathfrak{p}\right)\left(\operatorname{Ad}\left(w_{i}\right) \mathfrak{n}\right) \oplus U\left(\operatorname{Ad}\left(w_{i}\right) \mathfrak{l}\right)$. Hence we may assume that $c_{\mathbf{k}}^{(p)} \in$ $U\left(\operatorname{Ad}\left(w_{i}\right) \mathfrak{p}\right)\left(\operatorname{Ad}\left(w_{i}\right) \mathfrak{n}\right)$ or $c_{\mathbf{k}}^{(p)} \in U\left(\operatorname{Ad}\left(w_{i}\right) \mathfrak{l}\right)$. If $c_{\mathbf{k}}^{(p)} \in U\left(\operatorname{Ad}\left(w_{i}\right) \mathfrak{p}\right)\left(\operatorname{Ad}\left(w_{i}\right) \mathfrak{n}\right)$ then $\operatorname{Ad}\left(w_{i}\right)^{-1} c_{\mathbf{k}}^{(p)} u^{\prime}=0$ since $\mathfrak{n}$ acts on $J_{w_{i}^{-1}}^{\prime}\left(\sigma \otimes e^{\lambda+\rho}\right)$ trivially. If $c_{\mathbf{k}}^{(p)} \in U\left(\operatorname{Ad}\left(w_{i}\right) \mathfrak{l}\right)$ then $w_{i}^{-1}$ wt $\left.c_{\mathbf{k}}^{(p)}\right|_{\mathfrak{a}}=0$. Hence

$$
\begin{aligned}
w_{i}^{-1}\left(\operatorname{wt} b_{\mathbf{k}}^{(p)}+\right. & \left.\operatorname{wt}\left(R_{i}^{\prime}\left(a^{\prime}\right)\left(f f_{\mathbf{k}}\right)\right)\right)\left.\right|_{\mathfrak{a}} \\
& =\left.w_{i}^{-1}\left(\operatorname{wt} c_{\mathbf{k}}^{(p)}+\operatorname{wt} b_{\mathbf{k}}^{(p)}+\operatorname{wt} a^{\prime}+\operatorname{wt} f+\operatorname{wt} f_{\mathbf{k}}\right)\right|_{\mathfrak{a}} \\
& =\left.w_{i}^{-1}\left(\operatorname{wt} a_{\mathbf{k}}^{(p)}+\operatorname{wt} b_{\mathbf{k}}^{(p)}+\operatorname{wt} c_{\mathbf{k}}^{(p)}+\operatorname{wt} f+\operatorname{wt} f_{\mathbf{k}}-\operatorname{wt} a^{\prime \prime}\right)\right|_{\mathfrak{a}} \\
& =\left.w_{i}^{-1}\left(\operatorname{wt}\left(\left(\operatorname{ad}(e)^{\mathbf{k}} X\right) E\right)+\operatorname{wt} f+\operatorname{wt} f_{\mathbf{k}}-\operatorname{wt} a^{\prime \prime}\right)\right|_{\mathfrak{a}} \\
& =\left.w_{i}^{-1}\left(\operatorname{wt} X+\operatorname{wt} E+\operatorname{wt} f-\operatorname{wt} a^{\prime \prime}\right)\right|_{\mathfrak{a}} \\
& =\nu+\left.w_{i}^{-1}\left(\operatorname{wt} X-\operatorname{wt} a^{\prime \prime}\right)\right|_{\mathfrak{a}}>\nu+\left.w_{i}^{-1} \operatorname{wt} X\right|_{\mathfrak{a}} .
\end{aligned}
$$

So we have

$$
\begin{aligned}
& \delta_{i}\left(b_{\mathbf{k}}^{(p)}, R_{i}^{\prime}\left(\left(a_{\mathbf{k}}^{(p)}\right)^{\vee}\right)\left(f f_{\mathbf{k}} \eta_{i}^{-1}\right), \operatorname{Ad}\left(w_{i}\right)^{-1} c_{\mathbf{k}}^{(p)} u^{\prime}\right) \\
& \quad-\delta_{i}\left(b_{\mathbf{k}}^{(p)}, R_{i}^{\prime}\left(\left(a_{\mathbf{k}}^{(p)}\right)^{\vee}\right)\left(f f_{\mathbf{k}}\right), \operatorname{Ad}\left(w_{i}\right)^{-1} c_{\mathbf{k}}^{(p)} u^{\prime}\right) \widetilde{\eta}_{i}^{-1} \in \sum_{\nu^{\prime}>\nu} V\left(\nu^{\prime}+w_{i}^{-1} \text { wt }\left.X\right|_{\mathfrak{a}}\right) \widetilde{\eta}_{i}^{-1} .
\end{aligned}
$$


Therefore,

$$
\begin{aligned}
X \delta_{i}(E, & \left.f \eta_{i}^{-1}, u^{\prime}\right)+\sum_{\nu^{\prime}>\nu} V\left(\nu^{\prime}+\left.w_{i}^{-1} \mathrm{wt} X\right|_{\mathfrak{a}}\right) \widetilde{\eta}_{i}^{-1} \\
& \in \sum_{\mathbf{k}, p} \delta_{i}\left(b_{\mathbf{k}}^{(p)}, R_{i}^{\prime}\left(\left(a_{\mathbf{k}}^{(p)}\right)^{\vee}\right)\left(f f_{\mathbf{k}}\right), \operatorname{Ad}\left(w_{i}\right)^{-1}\left(c_{\mathbf{k}}^{(p)}\right) u^{\prime}\right) \widetilde{\eta}_{i}^{-1} \\
& =\sum_{\mathbf{k}, p} \delta_{i}\left(a_{\mathbf{k}}^{(p)} b_{\mathbf{k}}^{(p)} c_{\mathbf{k}}^{(p)}, f f_{\mathbf{k}}, u^{\prime}\right) \widetilde{\eta}_{i}^{-1}+\sum_{\nu^{\prime}>\nu} V\left(\nu^{\prime}+\left.w_{i}^{-1} \mathrm{wt} X\right|_{\mathfrak{a}}\right) \widetilde{\eta}_{i}^{-1} \\
& =\sum_{\mathbf{k}} \delta_{i}\left(\operatorname{ad}(e)^{\mathbf{k}}(X) E, f f_{\mathbf{k}}, u^{\prime}\right) \widetilde{\eta}_{i}^{-1}+\sum_{\nu^{\prime}>\nu} V\left(\nu^{\prime}+\left.w_{i}^{-1} \mathrm{wt} X\right|_{\mathfrak{a}}\right) \widetilde{\eta}_{i}^{-1} \\
& =\left(X \delta_{i}\left(E, f, u^{\prime}\right)\right) \widetilde{\eta}_{i}^{-1}+\sum_{\nu^{\prime}>\nu} V\left(\nu^{\prime}+\left.w_{i}^{-1} \mathrm{wt} X\right|_{\mathfrak{a}}\right) \widetilde{\eta}_{i}^{-1} .
\end{aligned}
$$

Proposition 8.7. Let $\widetilde{\mu} \in(\mathfrak{h} \cap \mathfrak{m})^{*}$ be the infinitesimal character of $\sigma$. Assume that $I_{i} / I_{i-1} \neq 0$ and for all $\widetilde{w} \in \widetilde{W}$,

$$
\lambda-\left.\left.\widetilde{w}(\lambda+\widetilde{\mu})\right|_{\mathfrak{a}} \notin \mathbb{Z}_{\leq 0}\left(\left(\Sigma^{+} \backslash \Sigma_{M}^{+}\right) \cap w_{i}^{-1} \Sigma^{+}\right)\right|_{\mathfrak{a}} \backslash\{0\} .
$$

Then

$$
\mathrm{Wh}_{\eta}\left(I_{i}^{\prime}\right)=\left\{\left(\eta_{i}^{-1} \otimes u^{\prime}\right) \delta_{i} \mid u^{\prime} \in \mathrm{Wh}_{w_{i}^{-1} \eta}\left(\left(\sigma \otimes e^{\lambda+\rho}\right)^{\prime}\right)\right\} .
$$

Proof. Let $x=\sum_{s} \delta_{i}\left(E_{s}, f_{s} \eta_{i}^{-1}, u_{s}^{\prime}\right) \in \mathrm{Wh}_{\eta}\left(I_{i}^{\prime}\right)$ where $E_{s} \in U\left(\operatorname{Ad}\left(w_{i}\right) \overline{\mathfrak{n}} \cap \overline{\mathfrak{n}_{0}}\right)$, $f_{s} \in \mathcal{P}\left(O_{i}\right)$ and $u_{s}^{\prime} \in J_{w_{i}^{-1} \eta}^{\prime}\left(\sigma \otimes e^{\lambda+\rho}\right)$. By Lemma 3.5, we have $(X-\eta(X)) x=$ $\sum_{s} \delta_{i}\left(E_{s}, L(X)\left(f_{s}\right) \eta_{i}^{-1}, u_{s}^{\prime}\right)$ for $X \in \operatorname{Ad}\left(w_{i}\right) \overline{\mathfrak{n}} \cap \mathfrak{n}_{0}$. Hence, we may assume $f_{s}=1$.

Let $z \in Z(\mathfrak{g})$. Since $J_{\eta}^{\prime}(I(\sigma, \lambda))$ has infinitesimal character $-(\lambda+\widetilde{\mu}), I_{i}^{\prime}$ has the same character. Let $\chi(z)$ be a complex number such that $z$ acts by $\chi(z)$ on $I_{i}^{\prime}$. Take $E_{s}$ and $u_{s}^{\prime}$ such that $E_{s}$ are $\mathfrak{a}_{0}$-weight vectors and $\left\{E_{s}\right\}$ is linearly independent. Let $\nu=\min \left\{w_{i}^{-1} \text { wt }\left.E_{s}\right|_{\mathfrak{a}}\right\}_{s}$.

Since $\gamma_{2} \gamma_{1}(z)-\gamma_{3} \gamma_{2} \gamma_{1}(z) \in\left(\operatorname{Ad}\left(w_{i}\right) \mathfrak{n}\right) U\left(\operatorname{Ad}\left(w_{i}\right) \mathfrak{p}\right)$, we have

$$
\gamma_{2} \gamma_{1}(z) x-\gamma_{3} \gamma_{2} \gamma_{1}(z) x \in \sum_{\nu^{\prime}>\nu} V\left(\nu^{\prime}\right) \widetilde{\eta}_{i}^{-1}
$$

by Lemmas 8.5 and 8.6. By Lemma 8.6,

$$
\gamma_{3} \gamma_{2} \gamma_{1}(z) x \in\left(\gamma_{3} \gamma_{2} \gamma_{1}(z) \sum_{w_{i}^{-1} \text { wt }\left.E_{s}\right|_{\mathfrak{a}}=\nu} \delta_{i}\left(E_{s}, 1, u_{s}^{\prime}\right)\right) \widetilde{\eta}_{i}^{-1}+\sum_{\nu^{\prime}>\nu} V\left(\nu^{\prime}\right) \widetilde{\eta}_{i}^{-1} .
$$

Therefore,

$$
\begin{aligned}
\chi(z) x=z x & =\gamma_{2} \gamma_{1}(z) x \\
\in & \left(\gamma_{3} \gamma_{2} \gamma_{1}(z) \sum_{w_{i}^{-1} \text { wt }\left.E_{s}\right|_{\mathfrak{a}}=\nu} \delta_{i}\left(E_{s}, 1, u_{s}^{\prime}\right)\right) \widetilde{\eta}_{i}^{-1}+\sum_{\nu^{\prime}>\nu} V\left(\nu^{\prime}\right) \widetilde{\eta}_{i}^{-1} .
\end{aligned}
$$


By Lemma $8.6(X=1)$, we have

$$
x \in \sum_{w_{i}^{-1} \text { wt }\left.E_{s}\right|_{\mathfrak{a}}=\nu} \delta_{i}\left(E_{s}, 1, u_{s}^{\prime}\right) \widetilde{\eta}_{i}^{-1}+\sum_{\nu^{\prime}>\nu} V\left(\nu^{\prime}\right) \widetilde{\eta}_{i}^{-1} .
$$

Hence

$$
\left(\left(\chi(z)-\gamma_{3} \gamma_{2} \gamma_{1}(z)\right)\left(\sum_{\left.w_{i}^{-1} \operatorname{wt} E_{s}\right|_{\mathfrak{a}}=\nu} \delta_{i}\left(E_{s}, 1, u_{s}^{\prime}\right)\right)\right) \widetilde{\eta}_{i}^{-1} \in \sum_{\nu^{\prime}>\nu} V\left(\nu^{\prime}\right) \widetilde{\eta}_{i}^{-1} .
$$

By Lemma 8.5, the left hand side is in $V(\nu){\widetilde{\eta_{i}}}^{-1}$. Hence

$$
\left(\chi(z)-\gamma_{3} \gamma_{2} \gamma_{1}(z)\right) \delta_{i}\left(E_{s}, 1, u_{s}^{\prime}\right)=0
$$

for all $s$ such that $w_{i}^{-1}$ wt $\left.E_{s}\right|_{\mathfrak{a}}=\nu$. By the same calculation as in the proof of Lemma 2.8, $H \delta_{i}\left(E_{s}, 1, u_{s}^{\prime}\right)=\left(-w_{i} \lambda+\right.$ wt $\left.E_{s}+\rho_{0}\right)(H) \delta_{i}\left(E_{s}, 1, u_{s}^{\prime}\right)$ for $H \in \operatorname{Ad}\left(w_{i}\right) \mathfrak{a}$. By Lemma 8.2 , there exists $\widetilde{w} \in \widetilde{W}$ such that $-\left.\widetilde{w}(\lambda+\widetilde{\mu})\right|_{\operatorname{Ad}\left(w_{i}\right) \mathfrak{a}}=-w_{i} \lambda+$ wt $E_{s}$. Then $\lambda-\left.w_{i}^{-1} \widetilde{w}(\lambda+\widetilde{\mu})\right|_{\mathfrak{a}}=w_{i}^{-1}$ wt $\left.\left.E_{s}\right|_{\mathfrak{a}} \in \mathbb{Z}_{\leq 0}\left(\left(\Sigma^{+} \backslash \Sigma_{M}^{+}\right) \cap w_{i}^{-1} \Sigma^{+}\right)\right|_{\mathfrak{a}}$. By assumption, $w_{i}^{-1}$ wt $\left.E_{s}\right|_{\mathfrak{a}}=0$, i.e., $E_{s} \in \mathbb{C}$. Hence, we may assume that $x$ has the form $x=\delta_{i}\left(1, \eta_{i}^{-1}, u^{\prime}\right)+\sum_{s \geq 2} \delta_{i}\left(E_{s}, \eta_{i}^{-1}, u_{s}^{\prime}\right)$ where $E_{s} \notin \mathbb{C}$ for all $s \geq 2$.

Take $X \in \mathfrak{n}_{0} \cap \operatorname{Ad}\left(w_{i}\right) \mathfrak{m}$. Then by Lemmas 3.5 and 8.6 ,

$$
0=(X-\eta(X)) x \in \delta_{i}\left(1,1,\left(\operatorname{Ad}\left(w_{i}\right)^{-1} X-\eta(X)\right) u^{\prime}\right) \widetilde{\eta}_{i}^{-1}+\sum_{\nu^{\prime}>0} V\left(\nu^{\prime}\right) \widetilde{\eta}_{i}^{-1} .
$$

Therefore, $\delta_{i}\left(1,1,\left(\operatorname{Ad}\left(w_{i}\right)^{-1} X-\eta(X)\right) u^{\prime}\right)=0$. Hence $u^{\prime} \in \mathrm{Wh}_{w_{i}^{-1} \eta}\left(\left(\sigma \otimes e^{\lambda+\rho}\right)^{\prime}\right)$. This implies that $x-\delta_{i}\left(1, \eta_{i}^{-1}, u^{\prime}\right) \in \mathrm{Wh}_{\eta}\left(I_{i}^{\prime}\right)$. If $x-\delta_{i}\left(1, \eta_{i}^{-1}, u^{\prime}\right) \neq 0$, then $\min \left\{w_{i}^{-1} \text { wt }\left.E_{s}\right|_{\mathfrak{a}}\right\}_{s \geq 2}=0$ by the above argument. This is a contradiction.

Theorem 8.8. Assume that for all $w \in W(M)$ with $w\left(\Sigma^{+} \backslash \Sigma_{M}^{+}\right) \cap \operatorname{supp} \eta=\emptyset$, the following two conditions hold:

(a) $\langle\check{\alpha}, \lambda+\nu\rangle \notin \mathbb{Z}_{\leq 0}$ for each exponent $\nu$ of $\sigma$ and $\alpha \in \Sigma^{+} \backslash w^{-1}\left(\Sigma^{+} \cup \Sigma_{\eta}^{-}\right)$.

(b) $\lambda-\left.\left.\widetilde{w}(\lambda+\widetilde{\mu})\right|_{\mathfrak{a}} \notin \mathbb{Z}_{\leq 0}\left(\left(\Sigma^{+} \backslash \Sigma_{M}^{+}\right) \cap w^{-1} \Sigma^{+}\right)\right|_{\mathfrak{a}} \backslash\{0\}$ for all $\widetilde{w} \in \widetilde{W}$, where $\widetilde{\mu}$ is the infinitesimal character of $\sigma$.

Moreover, assume that $\eta$ is unitary. Then

$$
\operatorname{dim} \mathrm{Wh}_{\eta}\left(I(\sigma, \lambda)^{\prime}\right)=\sum_{w \in W(M), w\left(\Sigma^{+} \backslash \Sigma_{M}^{+}\right) \cap \operatorname{supp} \eta=\emptyset} \operatorname{dim} \mathrm{Wh}_{w^{-1} \eta}\left(\left(\sigma \otimes e^{\lambda+\rho}\right)^{\prime}\right) .
$$

Remark 8.9. We have $w\left(\Sigma^{+} \backslash \Sigma_{M}^{+}\right) \cap \operatorname{supp} \eta=\emptyset$ if and only if $\eta$ is trivial on $w N w^{-1} \cap N_{0}$. About this condition, see Theorem 4.7. 
Proof of Theorem 8.8. By the exact sequence $0 \rightarrow I_{i-1} \rightarrow I_{i} \rightarrow I_{i} / I_{i-1} \rightarrow 0$, we have $0 \rightarrow \mathrm{Wh}_{\eta}\left(I_{i-1}\right) \rightarrow \mathrm{Wh}_{\eta}\left(I_{i}\right) \rightarrow \mathrm{Wh}_{\eta}\left(I_{i} / I_{i-1}\right)$. By Proposition 8.7, it is sufficient to prove that the last $\operatorname{map} \mathrm{Wh}_{\eta}\left(I_{i}\right) \rightarrow \mathrm{Wh}_{\eta}\left(I_{i} / I_{i-1}\right)$ is surjective.

Take $x \in \mathrm{Wh}_{\eta}\left(I_{i}^{\prime}\right) \simeq \mathrm{Wh}_{\eta}\left(I_{i} / I_{i-1}\right)$. Then $x$ is $\left(\eta_{i}^{-1} \otimes u^{\prime}\right) \delta_{i}$ for some $u^{\prime} \in$ $\mathrm{Wh}_{w_{i}^{-1} \eta}\left(\sigma \otimes e^{\lambda+\rho}\right)$ by Proposition 8.7. By Lemma 4.5, there exists a distribution $x_{t} \in I_{i}(\lambda+t \rho)$ with a meromorphic parameter $t$ such that $\left.x_{t}\right|_{U_{i}}$ is holomorphic and $\left.\left(\left.x_{t}\right|_{U_{i}}\right)\right|_{t=0}=x$. Moreover, $(X-\eta(X)) x_{t}=0$ for $X \in \mathfrak{n}_{0}$. By Proposition 4.4 and (a), the distribution $x_{t}$ is holomorphic at $t=0$. (See the proof of Lemma 4.5.) Hence $\left.x_{0}\right|_{U_{i}}=x$, so $\mathrm{Wh}_{\eta}\left(I_{i}\right) \rightarrow \mathrm{Wh}_{\eta}\left(I_{i} / I_{i-1}\right)$ is surjective.

Next we consider the module $\mathrm{Wh}_{\eta}\left(\left(I(\sigma, \lambda)_{K \text {-finite }}\right)^{*}\right)$.

Lemma 8.10. Let $V$ be an object of the category $\mathcal{O}_{P_{0}}^{\prime}$. Then $C\left(H^{0}\left(\mathfrak{n}_{\eta}, V\right)\right)=$ $H^{0}\left(\mathfrak{n}_{\eta}, C(V)\right)$ where $H^{0}\left(\mathfrak{n}_{\eta}, V\right)=\left\{v \in V \mid \mathfrak{n}_{\eta} v=0\right\}$ is the 0-th $\mathfrak{n}_{\eta}$-cohomology.

Proof. This follows from (we use Proposition 7.2(4))

$$
\begin{aligned}
H^{0}\left(\mathfrak{n}_{\eta}, C(V)\right) & =H^{0}\left(\mathfrak{n}_{\eta}, D^{\prime}(V)^{*}\right)=\left(D^{\prime}(V) / \mathfrak{n}_{\eta} D^{\prime}(V)\right)^{*} \\
& =C D^{\prime}\left(D^{\prime}(V) / \mathfrak{n}_{\eta} D^{\prime}(V)\right)=C\left(H^{0}\left(\mathfrak{n}_{\eta}, D^{\prime}(V)^{*}\right)_{\mathfrak{h} \text {-finite }}\right) \\
& =C\left(H^{0}\left(\mathfrak{n}_{\eta}, D^{\prime} D^{\prime}(V)\right)\right)=C\left(H^{0}\left(\mathfrak{n}_{\eta}, V\right)\right) .
\end{aligned}
$$

By Proposition 7.1, we have

$$
\mathrm{Wh}_{\eta}\left(\left(I(\sigma, \lambda)_{K \text {-finite }}\right)^{*}\right)=\mathrm{Wh}_{\eta}\left(C\left(J^{*}(I(\sigma, \lambda))\right)\right) .
$$

By the above lemma,

$$
\begin{aligned}
\mathrm{Wh}_{\eta}\left(C\left(J^{*}(I(\sigma, \lambda))\right)\right) & =\mathrm{Wh}_{\left.\eta\right|_{\mathfrak{r}_{\eta} \cap \mathfrak{n}_{0}}}\left(H^{0}\left(\mathfrak{n}_{\eta}, C\left(J^{*}(I(\sigma, \lambda))\right)\right)\right) \\
& =\mathrm{Wh}_{\left.\eta\right|_{\mathfrak{r}_{\eta} \cap \mathfrak{n}_{0}}}\left(C\left(H^{0}\left(\mathfrak{n}_{\eta}, J^{*}(I(\sigma, \lambda))\right)\right) .\right.
\end{aligned}
$$

Since $\eta \mid \mathfrak{r}_{\eta} \cap \mathfrak{n}_{0}$ is nondegenerate, a theorem of Lynch [Lyn79] shows that the dimension of the above space is determined by the character of $H^{0}\left(\mathfrak{n}_{\eta}, J^{*}(I(\sigma, \lambda))\right)$. To calculate $H^{0}\left(\mathfrak{n}_{\eta}, J^{*}(I(\sigma, \lambda))\right)$, we use the following lemma.

Lemma 8.11. Let $e_{1}, \ldots, e_{l}$ be a basis of $\operatorname{Ad}\left(w_{i}\right) \overline{\mathfrak{n}} \cap \mathfrak{n}_{0}$ such that each $e_{s}$ is a root vector and $\bigoplus_{s \leq t-1} \mathbb{C} e_{s}$ is an ideal of $\bigoplus_{s \leq t} \mathbb{C} e_{s}$. In $S_{w_{i}, 0}$, where 0 is the trivial representation of $\operatorname{Ad}\left(w_{i}\right) \overline{\mathfrak{n}} \cap \mathfrak{n}_{0}$, we have the following formulas:

(1) For all $t=1, \ldots, l$,

$$
\begin{aligned}
e_{t}\left(e_{1}^{-1} \cdots e_{t-1}^{-1} e_{t}^{-\left(k_{t}+1\right)} e_{t+1}^{-\left(k_{t+1}+1\right)} \cdots e_{l}^{-\left(k_{l}+1\right)}\right) & \\
& =e_{1}^{-1} \cdots e_{t-1}^{-1} e_{t}^{-k_{t}} e_{t+1}^{-\left(k_{t+1}+1\right)} \cdots e_{l}^{-\left(k_{l}+1\right)} .
\end{aligned}
$$


(2) Fix $t \in\{1, \ldots, l\}$ such that $e_{t} \in \mathfrak{n}_{\eta}$. Assume that $k_{s}=0$ for all $s<t$ such that $e_{s} \in \mathfrak{n}_{\eta}$. Then

$$
e_{t}\left(e_{1}^{-\left(k_{1}+1\right)} \cdots e_{l}^{-\left(k_{l}+1\right)}\right)=e_{1}^{-\left(k_{1}+1\right)} \ldots e_{t-1}^{-\left(k_{t-1}+1\right)} e_{t}^{-k_{t}} e_{t+1}^{-\left(k_{t}+1\right)} \ldots e_{l}^{-\left(k_{l}+1\right)} .
$$

(3) $X\left(e_{1}^{-1} \cdots e_{l}^{-1}\right)=\left(e_{1}^{-1} \cdots e_{l}^{-1}\right) X$ for $X \in \operatorname{Ad}\left(w_{i}\right) \mathfrak{m} \cap \mathfrak{n}_{0}$.

Proof. Let $\alpha_{s}$ be the restricted root corresponding to $e_{s}$.

(1) It is sufficient to prove the equality $e_{t}\left(e_{1}^{-1} \cdots e_{t-1}^{-1}\right)=\left(e_{1}^{-1} \cdots e_{t-1}^{-1}\right) e_{t}$ in $S_{e_{1}} \otimes_{U(\mathfrak{g})} \cdots \otimes_{U(\mathfrak{g})} S_{e_{t-1}}$. Since $\bigoplus_{s=1}^{t-1} \mathbb{C} e_{s}$ is an ideal of $\bigoplus_{s=1}^{t} \mathbb{C} e_{s}$, we have

$$
e_{t}\left(e_{1}^{-1} \cdots e_{t-1}^{-1}\right)-\left(e_{1}^{-1} \cdots e_{t-1}^{-1}\right) e_{t} \in \bigoplus_{p_{s} \geq 0} \mathbb{C} e_{1}^{-\left(p_{1}+1\right)} \cdots e_{t-1}^{-\left(p_{t-1}+1\right)} .
$$

The $\mathfrak{a}_{0}$-weight of the left hand side is $-\alpha_{1}-\cdots-\alpha_{t-1}+\alpha_{t}$. However, the set of $\mathfrak{a}_{0}$-weights of the right hand side is $\left\{-\left(p_{1}+1\right) \alpha_{1}-\cdots-\left(p_{t-1}+1\right) \alpha_{t-1} \mid p_{s} \in \mathbb{Z}_{\geq 0}\right\}$. Hence each $\mathfrak{a}_{0}$-weight appearing in the right hand side is less than that of the left hand side. This implies $e_{t}\left(e_{1}^{-1} \ldots e_{t-1}^{-1}\right)-\left(e_{1}^{-1} \ldots e_{t-1}^{-1}\right) e_{t}=0$.

(2) We will prove $e_{t}\left(e_{1}^{-\left(k_{1}+1\right)} \cdots e_{t-1}^{-\left(k_{t-1}+1\right)}\right)=\left(e_{1}^{-\left(k_{1}+1\right)} \ldots e_{t-1}^{-\left(k_{t-1}+1\right)}\right) e_{t}$ in $S_{e_{1}} \otimes_{U(\mathfrak{g})} \cdots \otimes_{U(\mathfrak{g})} S_{e_{t-1}}$. As in the proof of (1), we have

$$
\begin{aligned}
e_{t}\left(e_{1}^{-\left(k_{1}+1\right)} \cdots e_{t-1}^{-\left(k_{t-1}+1\right)}\right)-\left(e_{1}^{-\left(k_{1}+1\right)} \cdots\right. & \left.e_{t-1}^{-\left(k_{t-1}+1\right)}\right) e_{t} \\
& \in \bigoplus_{p_{s} \geq 0} \mathbb{C} e_{1}^{-\left(p_{1}+1\right)} \cdots e_{t-1}^{-\left(p_{t-1}+1\right)} .
\end{aligned}
$$

The $\mathfrak{a}_{\eta}$-weight of the left hand side is $\sum_{e_{s} \in \mathfrak{n}_{\eta}, s<t}-\alpha_{s}+\alpha_{t}$. However, the set of $\mathfrak{a}_{\eta}$-weights of the right hand side is $\left\{\sum_{e_{s} \in \mathfrak{n}_{\eta}, s<t}-\left(p_{s}+1\right) \alpha_{s} \mid p_{s} \in \mathbb{Z}_{\geq 0}\right\}$. Hence each $\mathfrak{a}_{\eta}$-weight appearing in the right hand side is less than that of the left hand side. This implies the assertion.

(3) We may assume $X$ is a restricted root vector. Let $\alpha$ be the restricted root corresponding to $X$. Since $X$ normalizes $\operatorname{Ad}\left(w_{i}\right) \overline{\mathfrak{n}} \cap \mathfrak{n}_{0}$, we have

$$
X\left(e_{1}^{-1} \cdots e_{l}^{-1}\right)-\left(e_{1}^{-1} \cdots e_{l}^{-1}\right) X \in \bigoplus_{p_{s} \geq 0} \mathbb{C} e_{1}^{-\left(p_{1}+1\right)} \cdots e_{l}^{-\left(p_{l}+1\right)} .
$$

Then $X\left(e_{1}^{-1} \cdots e_{l}^{-1}\right)-\left(e_{1}^{-1} \cdots e_{l}^{-1}\right) X$ has the $\mathfrak{a}_{0}$-weight $-\left(\alpha_{1}+\cdots+\alpha_{s}\right)+\alpha$. However, $e_{1}^{-\left(p_{1}+1\right)} \cdots e_{l}^{-\left(p_{l}+1\right)}$ has the $\mathfrak{a}_{0}$-weight $-\left(\left(p_{1}+1\right) \alpha_{1}+\cdots+\left(p_{l}+1\right) \alpha_{l}\right)$. If $-\left(\left(p_{1}+1\right) \alpha_{1}+\cdots+\left(p_{l}+1\right) \alpha_{l}\right)=-\left(\alpha_{1}+\cdots+\alpha_{s}\right)+\alpha$, then $\left(\left(p_{1}+1\right) \alpha_{1}+\cdots+\right.$ $\left.\left(p_{l}+1\right) \alpha_{l}\right)\left.\right|_{\operatorname{Ad}\left(w_{i}\right) \mathfrak{a}}=\left.\left(\alpha_{1}+\cdots+\alpha_{l}\right)\right|_{\operatorname{Ad}\left(w_{i}\right) \mathfrak{a}}$. Hence $p_{1}=\cdots=p_{l}=0$. Therefore, $\alpha=0$, a contradiction. Hence $X\left(e_{1}^{-1} \cdots e_{l}^{-1}\right)-\left(e_{1}^{-1} \cdots e_{l}^{-1}\right) X=0$.

Lemma 8.12. Let $e_{1}, \ldots, e_{l}$ be a basis of $\operatorname{Ad}\left(w_{i}\right) \overline{\mathfrak{n}} \cap \mathfrak{n}_{0}$ such that each $e_{s}$ is a root vector and $\bigoplus_{s \leq t-1} \mathbb{C} e_{s}$ is an ideal of $\bigoplus_{s \leq t} \mathbb{C} e_{s}$. Let $V$ be a $U(\mathfrak{m} \oplus \mathfrak{a})$ representation. Regard $V$ as a p-representation by $\mathfrak{n} V=0$. By Lemma 6.3, 
$T_{w_{i}}\left(U(\mathfrak{g}) \otimes_{U(\mathfrak{p})} V\right) \simeq\left(\bigoplus_{k_{s} \geq 0} \mathbb{C} e_{1}^{-\left(k_{1}+1\right)} \cdots e_{l}^{-\left(k_{l}+1\right)}\right) \otimes U\left(\operatorname{Ad}\left(w_{i}\right) \overline{\mathfrak{n}} \cap \overline{\mathfrak{n}_{0}}\right) \otimes w_{i} V$. Then $\left\{v \in e_{1}^{-1} \cdots e_{l}^{-1} \otimes 1 \otimes w_{i} V \mid \mathfrak{n}_{\eta} v=0\right\}=e_{1}^{-1} \cdots e_{l}^{-1} \otimes 1 \otimes H^{0}\left(\operatorname{Ad}\left(w_{i}\right) \mathfrak{m} \cap \mathfrak{n}_{\eta}, w_{i} V\right)$.

Proof. Take $v=e_{1}^{-1} \cdots e_{l}^{-1} \otimes 1 \otimes v_{0} \in H^{0}\left(\mathfrak{n}_{\eta}, T_{w_{i}}\left(U(\mathfrak{g}) \otimes_{U(\mathfrak{p})} V\right)\right)$. Then for $X \in$ $\operatorname{Ad}\left(w_{i}\right) \mathfrak{m} \cap \mathfrak{n}_{\eta}$ we have $X\left(e_{1}^{-1} \cdots e_{l}^{-1} \otimes 1 \otimes v_{0}\right)=0$. By Lemma 8.11, we have $e_{1}^{-1} \cdots e_{l}^{-1} \otimes 1 \otimes X v_{0}=0$. Hence $X v_{0}=0$.

By the definition of the Harish-Chandra homomorphism, we get the following.

Lemma 8.13. Let $\mathfrak{q}$ be a parabolic subalgebra of $\mathfrak{g}$ containing $\mathfrak{h} \oplus \mathfrak{u}_{0}$. Take the Levi decomposition $\mathfrak{l}_{\mathfrak{q}} \oplus \mathfrak{u}_{\mathfrak{q}}$ of $\mathfrak{q}$ such that $\mathfrak{h} \subset \mathfrak{l}_{\mathfrak{q}}$. Let $\widetilde{W_{\mathfrak{l}_{\mathfrak{q}}}} \subset \widetilde{W}$ be the Weyl group of $\mathfrak{l}_{\mathfrak{q}}$, and $V$ a $\mathfrak{g}$-module with infinitesimal character $\widetilde{\mu}$. Put $V^{\prime}=H^{0}\left(\mathfrak{u}_{\mathfrak{q}}, V\right)$ and $\widetilde{\rho_{\mathfrak{u}_{\mathfrak{q}}}}(H)=\left.(1 / 2) \operatorname{Tr} \operatorname{ad}(H)\right|_{\mathfrak{u}_{\mathfrak{q}}}$ for $H \in \mathfrak{h}$. Then $V^{\prime}$ is $\mathfrak{l}_{\mathfrak{q}}$-stable and $V^{\prime}=$ $\bigoplus_{\widetilde{w} \in \widetilde{W_{\mathfrak{l}_{\mathfrak{q}}}} \backslash \widetilde{W}}\left(V^{\prime}\right)_{\left[\widetilde{w} \widetilde{\mu}-\widetilde{\rho_{\mathfrak{u}_{\mathfrak{q}}}}\right]}$ where $\left(V^{\prime}\right)_{\left[\widetilde{w} \widetilde{\mu}-\widetilde{\rho_{\mathfrak{u}_{\mathfrak{q}}}}\right]}$ is the maximal $\mathfrak{l}_{\mathfrak{q}}$-submodule whose infinitesimal character is $\widetilde{w} \widetilde{\mu}-\widetilde{\rho_{\mathfrak{u}_{\mathfrak{q}}}}$. In particular, for every $\mathfrak{l}_{\mathfrak{q}}$-submodule $V^{\prime \prime}$ of $V^{\prime}$, all highest weights of $V^{\prime} / V^{\prime \prime}$ belong to $\{\widetilde{w} \widetilde{\mu}-\widetilde{\rho} \mid \widetilde{w} \in \widetilde{W}\}$.

The following lemma is well-known.

Lemma 8.14. Let $V \in \mathcal{O}_{P_{0}}^{\prime}$. Assume that $V$ has infinitesimal character $\widetilde{\lambda} \in \mathfrak{h}^{*}$. Then all $\mathfrak{h}$-weights appearing in $V$ belong to $\left\{\widetilde{w} \widetilde{\lambda}-\widetilde{\rho}-\alpha \mid \widetilde{w} \in \widetilde{W}, \alpha \in \mathbb{Z}_{\geq 0} \Delta^{+}\right\}$.

Take a filtration $\widetilde{I}_{i} \subset J_{\eta}^{*}(I(\sigma, \lambda))$ as in Theorem 7.5. Now we determine the dimension of the space of Whittaker vectors of $\widetilde{I}_{i} / \widetilde{I_{i-1}}$ under some conditions.

Lemma 8.15. Let $\widetilde{\mu}$ be the infinitesimal character of $\sigma$. Assume that $(\lambda+\widetilde{\mu})-$ $\widetilde{w}(\lambda+\widetilde{\mu}) \notin \mathbb{Z} \Delta$ for all $\widetilde{w} \in \widetilde{W} \backslash \widetilde{W_{M}}$. Then

$$
\operatorname{dim} \mathrm{Wh}_{\eta}\left(T_{w_{i}}\left(U(\mathfrak{g}) \otimes_{U(\mathfrak{p})} J^{*}\left(\sigma \otimes e^{\lambda+\rho}\right)\right)\right)=\operatorname{dim}_{\mathrm{Wh}_{w_{i}^{-1} \eta}}\left(\left(\sigma_{M \cap K \text {-finite }}\right)^{*}\right) .
$$

Proof. Put $V=T_{w_{i}}\left(U(\mathfrak{g}) \otimes_{U(\mathfrak{p})} J^{*}\left(\sigma \otimes e^{\lambda+\rho}\right)\right)$. Let $e_{1}, \ldots, e_{l}$ be a basis of $\operatorname{Ad}\left(w_{i}\right) \overline{\mathfrak{n}}$ $\cap \mathfrak{n}_{0}$ such that $\bigoplus_{s \leq t-1} \mathbb{C} e_{s}$ is an ideal of $\bigoplus_{s \leq t} \mathbb{C} e_{s}$. Moreover, assume that each $e_{s}$ is a root vector. For $\mathbf{k}=\left(k_{1}, \ldots, k_{l}\right) \in \mathbb{Z}^{l}$, put $e^{\mathbf{k}}=e_{1}^{k_{1}} \cdots e_{l}^{k_{l}}$. Set $\mathbf{1}=$ $(1, \ldots, 1) \in \mathbb{Z}^{l}$. Then

$$
V=\bigoplus_{\mathbf{k} \in \mathbb{Z}_{\geq 0}^{l}} \mathbb{C} e^{-(\mathbf{k}+\mathbf{1})} \otimes U\left(\operatorname{Ad}\left(w_{i}\right) \overline{\mathfrak{n}} \cap \overline{\mathfrak{n}_{0}}\right) \otimes w_{i} J^{*}\left(\sigma \otimes e^{\lambda+\rho}\right) .
$$

Put

$$
V^{\prime}=\bigoplus_{\mathbf{k} \in \mathcal{A}} \mathbb{C} e^{-(\mathbf{k}+\mathbf{1})} \otimes U\left(\operatorname{Ad}\left(w_{i}\right) \overline{\mathfrak{n}} \cap \overline{\mathfrak{n}_{0}} \cap \mathfrak{m}_{\eta}\right) \otimes H^{0}\left(\mathfrak{m} \cap \mathfrak{n}_{\eta}, w_{i} J^{*}\left(\sigma \otimes e^{\lambda+\rho}\right)\right)
$$

where $\mathcal{A}=\left\{\left(k_{1}, \ldots, k_{l}\right) \in \mathbb{Z}_{\geq 0}^{l} \mid\right.$ if $e_{s} \in \mathfrak{n}_{\eta}$ then $\left.k_{i}=0\right\}$. It is easy to see that $V^{\prime}$ is $\mathfrak{m}_{\eta} \oplus \mathfrak{a}_{\eta}$-stable. By Lemma 8.11, $V^{\prime} \subset H^{0}\left(\mathfrak{n}_{\eta}, V\right)$. We first prove that $V^{\prime}=$ $H^{0}\left(\mathfrak{n}_{\eta}, V\right)$. 
It is sufficient to prove that there exists no highest weight vector in $H^{0}\left(\mathfrak{n}_{\eta}, V\right) / V^{\prime}$. Let $v \in H^{0}\left(\mathfrak{n}_{\eta}, V\right)$ be such that $\left(\mathfrak{m}_{\eta} \cap \mathfrak{u}\right) v \in V^{\prime}$.

First, we prove that $v \in e^{-\mathbf{1}} \otimes U\left(\operatorname{Ad}\left(w_{i}\right) \overline{\mathfrak{n}} \cap \overline{\mathfrak{n}_{0}}\right) \otimes w_{i} J^{*}\left(\sigma \otimes e^{\lambda+\rho}\right)+V^{\prime}$. Take $y_{\mathbf{k}} \in U\left(\operatorname{Ad}\left(w_{i}\right) \overline{\mathfrak{n}} \cap \overline{\mathfrak{n}_{0}}\right) \otimes w_{i} J^{*}\left(\sigma \otimes e^{\lambda+\rho}\right)$ such that $v=\sum_{\mathbf{k}} e^{-(\mathbf{k}+\mathbf{1})} \otimes y_{\mathbf{k}}$. We prove that if $k_{t} \neq 0$ and $e_{t} \in \mathfrak{n}_{\eta}$ then $y_{\mathbf{k}}=0$ by induction on $t$ where $\mathbf{k}=\left(k_{1}, \ldots, k_{l}\right)$. Put $\mathbf{1}_{t}=\left(\delta_{s t}\right)_{1 \leq s \leq l} \in \mathbb{Z}^{l}$ ( $\delta_{s t}$ is Kronecker's delta). By inductive hypothesis, for $s<t$ such that $e_{s} \in \mathfrak{n}_{\eta}$, if $y_{\mathbf{k}} \neq 0$ then $k_{s}=0$. By Lemma 8.11(2), we have $e_{t} v=\sum_{\mathbf{k} \in \mathbb{Z}_{\geq 0}^{l}} e^{-(\mathbf{k}+\mathbf{1})+\mathbf{1}_{t}} \otimes y_{\mathbf{k}}$. Since $v \in H^{0}\left(\mathfrak{n}_{\eta}, V\right)$, we have $e_{t} v=0$. Hence if $e^{-(\mathbf{k}+\mathbf{1})+\mathbf{1}_{t}} \neq 0$ then $y_{\mathbf{k}}=0$. Since $e^{-(\mathbf{k}+\mathbf{1})+\mathbf{1}_{t}}=0$ is equivalent to $k_{t}=0, k_{t} \neq 0$ implies $y_{\mathbf{k}}=0$.

We now prove that if $k_{t} \neq 0$ then $e^{-(\mathbf{k}+\mathbf{1})} \otimes y_{\mathbf{k}} \in V^{\prime}$ by induction on $t$. If $e_{t} \in \mathfrak{n}_{\eta}$ then this claim is already proved. We may assume that $e_{t} \in \mathfrak{m}_{\eta}$. Hence $e_{t} V^{\prime} \subset V^{\prime}$. By inductive hypothesis, if $k_{s} \neq 0$ for some $s<t$ then $e^{-(\mathbf{k}+\mathbf{1})} \otimes y_{\mathbf{k}} \in V^{\prime}$. Then $e_{t} v \in \sum_{\mathbf{k} \in \mathbb{Z}_{\geq 0}^{l}} e^{-(\mathbf{k}+\mathbf{1})+\mathbf{1}_{t}} \otimes y_{\mathbf{k}}+V^{\prime}$ by Lemma 8.11(1). Since $e_{t} v \in V^{\prime}$, we have $\sum_{\mathbf{k} \in \mathbb{Z}_{\geq 0}^{l}} e^{-(\mathbf{k}+\mathbf{1})+\mathbf{1}_{t}} \otimes y_{\mathbf{k}} \in V^{\prime}$. By the definition of $V^{\prime}$, if $e^{-(\mathbf{k}+\mathbf{1})+\mathbf{1}_{t}} \neq 0$ then $e^{-(\mathbf{k}+\mathbf{1})} \otimes y_{\mathbf{k}} \in V^{\prime}$. Notice that $e^{-(\mathbf{k}+\mathbf{1})+\mathbf{1}_{t}} \neq 0$ if and only if $k_{t} \neq 0$. Hence we get the claim.

We now prove $v \in V^{\prime}$. We may assume that $v$ is a weight vector with respect to $\mathfrak{h}$. We can take $\widetilde{w} \in \widetilde{W}$ such that $-\widetilde{w}(\lambda+\widetilde{\mu})-\widetilde{\rho}$ is the $\mathfrak{h}$-weight of $v$ by Lemma 8.13. Put $\widetilde{\rho_{M}}=\sum_{\alpha \in \Delta_{M}^{+}}(1 / 2) \alpha$. Since $J^{*}\left(\sigma \otimes e^{\lambda+\rho}\right)$ has infinitesimal character $-(\widetilde{\mu}+\lambda+\rho)$, all $\mathfrak{h}$-weights appearing in $J^{*}\left(\sigma \otimes e^{\lambda+\rho}\right)$ are contained in $\left\{-\widetilde{w}(\widetilde{\mu}+\lambda+\rho)-\widetilde{\rho_{M}}+\alpha \mid \widetilde{w} \in \widetilde{W_{M}}, \alpha \in \mathbb{Z} \Delta_{M}\right\}$ by Lemma 8.14. Since $\rho \in \mathfrak{a}^{*}$, we have $\widetilde{w} \rho=\rho$ for $\widetilde{w} \in \widetilde{W_{M}}$. Hence $-\widetilde{w} \rho-\widetilde{\rho_{M}}=-\rho-\widetilde{\rho_{M}}=-\widetilde{\rho}$. Notice that $w_{i} \widetilde{\rho}-\widetilde{\rho} \in \mathbb{Z} \Delta$. Therefore all $\mathfrak{h}$-weights appearing in $V$ belong to

$$
\begin{array}{r}
-w_{i} \widetilde{W_{M}}(\widetilde{\mu}+\lambda)-w_{i} \widetilde{\rho}+w_{i} \mathbb{Z}_{M}+\mathbb{Z}_{\geq 0}\left(w_{i} \Delta^{-} \cap \Delta^{-}\right)-\mathbb{Z}_{\geq 1}\left(w_{i} \Delta^{-} \cap \Delta^{+}\right) \\
\subset-w_{i} \widetilde{W_{M}}(\widetilde{\mu}+\lambda)-\widetilde{\rho}+\mathbb{Z} \Delta
\end{array}
$$

by Lemma 6.3 . This implies that for some $\widetilde{w^{\prime}} \in \widetilde{W_{M}}$, we have $\widetilde{w}(\widetilde{\mu}+\lambda)-\widetilde{w_{i}} \widetilde{w^{\prime}}(\lambda+\widetilde{\mu})$ $\in \mathbb{Z} \Delta$. By assumption we have $\widetilde{w} \in w_{i} \widetilde{W_{M}}$. This implies $(\operatorname{wt} v)\left(\operatorname{Ad}\left(w_{i}\right) H\right)=$ $-\left(\lambda(H)+w_{i}^{-1} \widetilde{\rho}(H)\right)$ for all $H \in \mathfrak{a}$ where wt $v$ is the $\mathfrak{h}$-weight of $v$.

Take $E_{p} \in U\left(\operatorname{Ad}\left(w_{i}\right) \overline{\mathfrak{n}} \cap \overline{\mathfrak{n}_{0}}\right)$ and $x_{p} \in w_{i} J^{*}\left(\sigma \otimes e^{\lambda+\rho}\right)$ such that $v \in \sum_{p} e^{-\mathbf{1}} \otimes$ $E_{p} \otimes x_{p}+V^{\prime}$. We may assume that $E_{p}$ and $x_{p}$ are $\mathfrak{h}$-weight vectors. We denote their $\mathfrak{h}$-weights by wt $E_{p}$ and wt $x_{p}$. Fix $H \in \mathfrak{a}$. Then $\alpha(H)=0$ for all $\alpha \in \Delta_{M}$. Since wt $x_{p} \in-w_{i}\left(\widetilde{W_{M}}(\widetilde{\mu}+\lambda+\rho)-\widetilde{\rho_{M}}+\mathbb{Z} \Delta_{M}\right),\left(\right.$ wt $\left.x_{p}\right)\left(\operatorname{Ad}\left(w_{i}\right) H\right)=-(\lambda+\rho)(H)$. Hence

$$
\begin{aligned}
(\operatorname{wt} v)\left(\operatorname{Ad}\left(w_{i}\right) H\right) & =\left(\operatorname{wt}\left(e^{-\mathbf{1}}\right)+\operatorname{wt}\left(E_{p}\right)+\operatorname{wt}\left(x_{p}\right)\right)\left(\operatorname{Ad}\left(w_{i}\right)(H)\right) \\
& =\left(\operatorname{wt}\left(e^{-\mathbf{1}}\right)\left(\operatorname{Ad}\left(w_{i}\right) H\right)+\left(\operatorname{wt} E_{p}\right)\left(\operatorname{Ad}\left(w_{i}\right) H\right)-(\lambda+\rho)(H) .\right.
\end{aligned}
$$


We calculate $\operatorname{wt}\left(e^{-\mathbf{1}}\right)\left(\operatorname{Ad}\left(w_{i}\right) H\right)$. By definition,

$$
\operatorname{wt}\left(e^{-\mathbf{1}}\right)\left(\operatorname{Ad}\left(w_{i}\right) H\right)=-\left.\operatorname{Tr} \operatorname{ad}\left(\operatorname{Ad}\left(w_{i}\right) H\right)\right|_{\operatorname{Ad}\left(w_{i}\right) \overline{\mathfrak{n}} \cap \mathfrak{n}_{0}} .
$$

Since $\operatorname{Ad}\left(w_{i}\right) \overline{\mathfrak{n}} \cap \mathfrak{n}_{0}=\operatorname{Ad}\left(w_{i}\right) \overline{\mathfrak{n}_{0}} \cap \mathfrak{n}_{0}$, we have

$$
\begin{aligned}
\left.\operatorname{Tr} \operatorname{ad}\left(\operatorname{Ad}\left(w_{i}\right) H\right)\right|_{\operatorname{Ad}\left(w_{i}\right) \overline{\mathfrak{n}} \cap \mathfrak{n}_{0}} & =\left.\operatorname{Tr} \operatorname{ad}\left(\operatorname{Ad}\left(w_{i}\right) H\right)\right|_{\operatorname{Ad}\left(w_{i}\right) \overline{\mathfrak{n}_{0}} \cap \mathfrak{n}_{0}} \\
& =\left.\operatorname{Tr} \operatorname{ad}(H)\right|_{\operatorname{Ad}\left(w_{i}\right)^{-1} \mathfrak{n}_{0} \cap \overline{\mathfrak{n}_{0}}}=\left(-\widetilde{\rho}+w_{i}^{-1} \widetilde{\rho}\right)(H) .
\end{aligned}
$$

Since $H \in \mathfrak{a}, \widetilde{\rho}(H)=\rho(H)$. Hence

$$
(\operatorname{wt} v)\left(\operatorname{Ad}\left(w_{i}\right) H\right)=\left(\operatorname{wt} E_{p}\right)\left(\operatorname{Ad}\left(w_{i}\right) H\right)-\left(\lambda+w_{i}^{-1} \widetilde{\rho}\right)(H) .
$$

We have already proved that $(\operatorname{wt} v)\left(\operatorname{Ad}\left(w_{i}\right) H\right)=-\left(\lambda+w_{i}^{-1} \widetilde{\rho}\right)(H)$. Therefore we get $\left(\right.$ wt $\left.E_{p}\right)\left(\operatorname{Ad}\left(w_{i}\right) H\right)=0$ for all $H \in \mathfrak{a}$. Since $E_{p} \in U\left(\operatorname{Ad}\left(w_{i}\right) \overline{\mathfrak{n}}\right)$, this implies $E_{p} \in \mathbb{C}$, i.e., there exist $v^{\prime} \in e^{-\mathbf{1}} \otimes 1 \otimes w_{i} J^{*}\left(\sigma \otimes e^{\lambda+\rho}\right)$ and $v^{\prime \prime} \in V^{\prime}$ such that $v=v^{\prime}+v^{\prime \prime}$. Therefore $\mathfrak{n}_{\eta}\left(v^{\prime}\right)=\mathfrak{n}_{\eta}\left(v-v^{\prime \prime}\right)=0$. Hence $v^{\prime} \in V^{\prime}$ by Lemma 8.12. Therefore $H^{0}\left(\mathfrak{n}_{\eta}, V\right)=V^{\prime}$.

We now prove the lemma. For an $\mathfrak{m}_{0} \oplus \mathfrak{a}_{0}$-module $\tau$ and a subalgebra $\mathfrak{c}$ of $\mathfrak{g}$ containing $\mathfrak{m}_{0} \oplus \mathfrak{a}_{0}$, put $M_{\mathfrak{c}}(\tau)=U(\mathfrak{c}) \otimes_{U\left(\mathfrak{c} \cap \overline{\mathfrak{p}_{0}}\right)}\left(\tau \otimes \rho^{\prime}\right)$ where $\mathfrak{c} \cap \overline{\mathfrak{n}_{0}}$ acts on $\tau \otimes \rho^{\prime}$ trivially and $\rho^{\prime}(H)=(1 / 2)\left(\operatorname{Tr}\left(\left.\operatorname{ad}(H)\right|_{\mathfrak{c} \cap \overline{\mathfrak{n}_{0}}}\right)\right)$ for $H \in \mathfrak{a}_{0}$.

We give some notation and facts about $\mathcal{O}_{P_{0}}^{\prime}$. All facts are well-known. For $\tilde{\lambda} \in \mathfrak{h}^{*}$ such that $\left.\widetilde{\lambda}\right|_{\mathfrak{m}_{0} \cap \mathfrak{h}}$ is a regular dominant integral, let $\sigma_{M_{0} A_{0}, \widetilde{\lambda}}$ be the finitedimensional representation of $M_{0} A_{0}$ with infinitesimal character $\widetilde{\lambda}$. Let $L^{\prime}$ be a Levi sugbgoup of a parabolic subgroup such that $M_{0} A_{0} \subset L^{\prime}$. Let $\operatorname{ch} V_{0}$ be the character of $V_{0} \in \mathcal{O}_{P_{0} \cap L^{\prime}, L^{\prime}}^{\prime}$ and $K_{0}\left(\mathcal{O}_{P_{0} \cap L^{\prime}, L^{\prime}}^{\prime}\right)$ the Grothendieck group of $\mathcal{O}_{P_{0} \cap L^{\prime}, L^{\prime}}^{\prime}$. Then we can define $\operatorname{ch} V_{0}$ for $V_{0} \in K_{0}\left(\mathcal{O}_{P_{0} \cap L^{\prime}, L^{\prime}}^{\prime}\right)$ (namely, ch is additive) and ch $V_{0}=$ ch $V_{1}$ if and only if $V_{0}=V_{1}$ for $V_{0}, V_{1} \in K_{0}\left(\mathcal{O}_{P_{0} \cap L^{\prime}, L^{\prime}}^{\prime}\right)$. A basis of $K_{0}\left(\mathcal{O}_{\overline{P_{0}} \cap L^{\prime}, L^{\prime}}^{\prime}\right)$ is given by $\left\{M_{\mathfrak{l}}\left(\sigma_{M_{0} A_{0}, \tilde{\lambda}}\right)\right\}$. Let $P^{\prime \prime}$ be a parabolic subgroup of $L^{\prime}$ containing $P_{0} \cap L^{\prime}$, $L^{\prime \prime}$ its Levi subgroup and $\mathfrak{n}^{\prime \prime}$ the nilpotent radical of the Lie algebra of $P^{\prime \prime}$. Then for $V_{0} \in \mathcal{O}_{P_{0} \cap L^{\prime}, L^{\prime}}^{\prime}$, we have $H^{0}\left(\mathfrak{n}^{\prime \prime}, V_{0}\right) \in \mathcal{O}_{P_{0} \cap L^{\prime \prime}, L^{\prime \prime}}^{\prime}$

By Remark 2.5, $\operatorname{Ad}\left(w_{i}\right)\left(\mathfrak{m} \cap \mathfrak{p}_{0}\right)=\operatorname{Ad}\left(w_{i}\right) \mathfrak{m} \cap \mathfrak{p}_{0} \subset \operatorname{Ad}\left(w_{i}\right) \mathfrak{m} \cap \mathfrak{p}_{\eta}$. Therefore, $\mathfrak{m} \cap \mathfrak{p}_{0} \subset \mathfrak{m} \cap \operatorname{Ad}\left(w_{i}\right)^{-1} \mathfrak{p}_{\eta}$. Hence $\mathfrak{m} \cap \operatorname{Ad}\left(w_{i}\right)^{-1} \mathfrak{p}_{\eta}$ is a parabolic subalgebra of $\mathfrak{m}$. Therefore, $H^{0}\left(\operatorname{Ad}\left(w_{i}\right)^{-1} \mathfrak{n}_{\eta} \cap \mathfrak{m}, J^{*}\left(\sigma \otimes e^{\lambda+\rho}\right)\right) \in \mathcal{O}_{P_{0} \cap M \cap w_{i}^{-1} M_{\eta} w_{i}, M \cap w_{i}^{-1} M_{\eta} w_{i}}^{\prime}$. Recall that we have a functor $w_{i}$. (It twists the action of $\mathfrak{g}$ by $w_{i}$.) Since $w_{i}\left(P_{0} \cap M \cap w_{i}^{-1} M_{\eta} w_{i}\right) w_{i}^{-1}=P_{0} \cap w_{i} M w_{i}^{-1} \cap M_{\eta}$ (Remark 2.5), we deduce that $w_{i}\left(\mathcal{O}_{P_{0} \cap M \cap w_{i}^{-1} M_{\eta} w_{i}, M \cap w_{i}^{-1} M_{\eta} w_{i}}^{\prime}\right)=\mathcal{O}_{P_{0} \cap w_{i} M w_{i}^{-1} \cap M_{\eta}, w_{i} M w_{i}^{-1} \cap M_{\eta}}^{\prime}$. (This follows from the definition.) Therefore,

$$
H^{0}\left(\mathfrak{n}_{\eta} \cap \operatorname{Ad}\left(w_{i}\right) \mathfrak{m}, w_{i} J^{*}\left(\sigma \otimes e^{\lambda+\rho}\right)\right) \in \mathcal{O}_{P_{0} \cap w_{i} M w_{i}^{-1} \cap M_{\eta}, w_{i} M w_{i}^{-1} \cap M_{\eta}}^{\prime} .
$$


Hence we can take $c_{\widetilde{\lambda}}$ such that

$\operatorname{ch} D^{\prime} H^{0}\left(\mathfrak{n}_{\eta} \cap \operatorname{Ad}\left(w_{i}\right) \mathfrak{m}, w_{i} J^{*}\left(\sigma \otimes e^{\lambda+\rho}\right)\right)=\sum_{\widetilde{\lambda}} c_{\widetilde{\lambda}} \operatorname{ch} M_{\left(\mathfrak{m}_{\eta} \cap \operatorname{Ad}\left(w_{i}\right) \mathfrak{m}\right)+\mathfrak{a}_{0}}\left(\sigma_{M_{0} A_{0}, \tilde{\lambda}}\right)$

Then it is straightforward to prove $\operatorname{ch} D^{\prime} V^{\prime}=\sum_{\widetilde{\lambda}} c_{\widetilde{\lambda}} \operatorname{ch} M_{\mathfrak{m}_{\eta} \oplus \mathfrak{a}_{\eta}}\left(\sigma_{M_{0} A_{0}, \widetilde{\lambda}}\right)$. By a result of Lynch [Lyn79], the functor $X \mapsto \mathrm{Wh}_{\left.\eta\right|_{\mathfrak{m}_{\eta} \cap \mathfrak{n}_{0}}}\left(X^{*}\right)$ from the category $\mathcal{O} \frac{\prime}{P_{0}} \cap M_{\eta}, M_{\eta}$ to the category of vector spaces is exact. Therefore,

$$
\operatorname{dim} \mathrm{Wh}_{\left.\eta\right|_{\mathfrak{m}_{\eta} \cap \mathfrak{n}_{0}}}\left(C\left(V^{\prime}\right)\right)=\sum_{\widetilde{\lambda}} c_{\widetilde{\lambda}} \operatorname{dim} \mathrm{Wh}_{\left.\eta\right|_{\mathfrak{m}_{\eta} \cap \mathfrak{n}_{0}}}\left(M_{\mathfrak{m}_{\eta} \oplus \mathfrak{a}_{\eta}}\left(\sigma_{M_{0} A_{0}, \widetilde{\lambda}}\right)^{*}\right) .
$$

Lynch also proved $\operatorname{dim} \mathrm{Wh}_{\left.\eta\right|_{\mathfrak{m}_{\eta} \cap \mathfrak{n}_{0}}}\left(M_{\mathfrak{m}_{\eta}}\left(\sigma_{M_{0} A_{0}, \widetilde{\lambda}}\right)^{*}\right)=\operatorname{dim} \sigma_{M_{0} A_{0}, \widetilde{\lambda}} \cdot$ Therefore, by Lemma 8.10 and $V^{\prime}=H^{0}\left(\mathfrak{n}_{\eta}, V\right)$,

$$
\begin{aligned}
\operatorname{dim} \mathrm{Wh}_{\eta}\left(\widetilde{I_{i}} / \widetilde{I_{i-1}}\right) & =\operatorname{dim} \mathrm{Wh}_{\eta}(C(V))=\operatorname{dim} \mathrm{Wh}_{\left.\eta\right|_{\mathfrak{m}_{\eta} \cap \mathfrak{n}_{0}}}\left(H^{0}\left(\mathfrak{n}_{\eta}, C(V)\right)\right) \\
& =\operatorname{dim} \mathrm{Wh}_{\left.\eta\right|_{\mathfrak{m}_{\eta} \cap \mathfrak{n}_{0}}}\left(C\left(V^{\prime}\right)\right)=\sum_{\widetilde{\lambda}} c_{\widetilde{\lambda}} \operatorname{dim} \sigma_{M_{0} A_{0}, \widetilde{\lambda}} .
\end{aligned}
$$

By the same argument,

$$
\begin{aligned}
\sum_{\tilde{\lambda}} c_{\tilde{\lambda}} \operatorname{dim} \sigma_{M_{0} A_{0}, \tilde{\lambda}} & =\sum_{\widetilde{\lambda}} c_{\widetilde{\lambda}} \operatorname{dim} \operatorname{Wh}_{\left.\eta\right|_{\mathfrak{m}_{\eta} \cap \operatorname{Ad}\left(w_{i}\right) \mathfrak{m} \cap \mathfrak{n}_{0}}}\left(M_{\left(\mathfrak{m}_{\eta} \cap \operatorname{Ad}\left(w_{i}\right) \mathfrak{m}\right)+\mathfrak{a}_{0}}\left(\sigma_{M_{0} A_{0}, \tilde{\lambda}}\right)^{*}\right) \\
& =\operatorname{dim}_{\left.W_{\eta}\right|_{\mathfrak{m}_{\eta} \cap \operatorname{Ad}\left(w_{i}\right) \mathfrak{m} \cap \mathfrak{n}_{0}}}\left(C H^{0}\left(\mathfrak{n}_{\eta} \cap \operatorname{Ad}\left(w_{i}\right) \mathfrak{m}, w_{i} J^{*}\left(\sigma \otimes e^{\lambda+\rho}\right)\right)\right) \\
& =\operatorname{dim} \operatorname{Wh}_{\left.\eta\right|_{\operatorname{Ad}\left(w_{i}\right) \mathfrak{m} \cap \mathfrak{n}_{0}}}\left(C\left(w_{i} J^{*}\left(\sigma \otimes e^{\lambda+\rho}\right)\right)\right) \\
& =\operatorname{dim} \operatorname{Wh}_{w_{i}^{-1} \eta}\left(C\left(J^{*}\left(\sigma \otimes e^{\lambda+\rho}\right)\right)\right) \\
& =\operatorname{dim} \operatorname{Wh}_{w_{i}^{-1} \eta}\left(\left(\sigma_{M \cap K \text {-finite }}\right)^{*}\right) .
\end{aligned}
$$

This implies the conclusion.

Theorem 8.16. Let $\widetilde{\mu}$ be an infinitesimal character of $\sigma$. Assume that $(\lambda+\widetilde{\mu})-$ $\widetilde{w}(\lambda+\widetilde{\mu}) \notin \mathbb{Z} \Delta$ for all $\widetilde{w} \in \widetilde{W} \backslash \widetilde{W_{M}}$. Then

$$
\operatorname{dim} \mathrm{Wh}_{\eta}\left(\left(I(\sigma, \lambda)_{K \text {-finite }}\right)^{*}\right)=\sum_{w \in W(M)} \operatorname{dim} \mathrm{Wh}_{w^{-1} \eta}\left(\left(\sigma_{M \cap K \text {-finite }}\right)^{*}\right) .
$$

Proof. Let $I_{i}$ be the Bruhat filtration of $J^{\prime}(I(\sigma, \lambda))=J^{*}(I(\sigma, \lambda))$. Since all $\mathfrak{h}$-weights appearing in $I_{i} / I_{i-1} \simeq T_{w_{i}}\left(U(\mathfrak{g}) \otimes_{U(\mathfrak{p})} J^{*}\left(\sigma \otimes e^{\lambda+\rho}\right)\right)$ belong to $\left\{-w_{i} \widetilde{w}(\lambda+\widetilde{\mu})-\widetilde{\rho}+\alpha \mid \widetilde{w} \in \widetilde{W_{M}}, \alpha \in \Delta\right\}$, we have

$$
\operatorname{wt}\left(I_{i} / I_{i-1}\right) \cap\left(\operatorname{wt}\left(I_{j} / I_{j-1}\right)+\mathbb{Z} \Delta\right)=\emptyset
$$

if $i \neq j$, where $\operatorname{wt}\left(I_{i} / I_{i-1}\right)$ is the set of $\mathfrak{h}$-weights in $I_{i} / I_{i-1}$. Therefore, the exact sequence $0 \rightarrow I_{i-1} \rightarrow I_{i} \rightarrow I_{i} / I_{i-1} \rightarrow 0$ splits by the block decomposition 
of $\mathcal{O}_{P_{0}}^{\prime}$. Hence $J_{\eta}^{*}(I(\sigma, \lambda))=\bigoplus_{i} \Gamma_{\eta}\left(C\left(T_{w_{i}}\left(U(\mathfrak{g}) \otimes_{U(\mathfrak{p})} J^{*}\left(\sigma \otimes e^{\lambda+\rho}\right)\right)\right)\right)$. Therefore, the conclusion follows from Lemma 8.15.

Finally we study the case where $\sigma$ is finite-dimensional. Then $\mathfrak{m} \cap \mathfrak{n}_{0}$ acts on $\sigma$ as nilpotent operators. Therefore, $\mathrm{Wh}_{w_{i}^{-1} \eta}\left(\sigma^{*}\right) \neq 0$ if and only if $w_{i}^{-1} \eta=0$ on $\mathfrak{m} \cap \mathfrak{n}_{0}$.

Definition 8.17. Let $\Theta, \Theta_{1}, \Theta_{2}$ be subsets of $\Pi$.

(1) Put $W(\Theta)=\left\{w \in W \mid w(\Theta) \subset \Sigma^{+}\right\}$and $\Sigma_{\Theta}=\mathbb{Z} \Theta \cap \Sigma$.

(2) Put $W\left(\Theta_{1}, \Theta_{2}\right)=\left\{w \in W\left(\Theta_{1}\right) \cap W\left(\Theta_{2}\right)^{-1} \mid w\left(\Sigma_{\Theta_{1}}\right) \cap \Sigma_{\Theta_{2}}=\emptyset\right\}$.

(3) Let $W_{\Theta}$ be the Weyl group of $\Sigma_{\Theta}$.

Lemma 8.18. Let $\Theta$ be the subset of $\Pi$ corresponding to $P$.

(1) $\# W(\operatorname{supp} \eta, \Theta)=\#\left\{w \in W(M) \mid w\left(\Sigma^{+}\right) \cap \Sigma_{\eta}^{+}=\emptyset\right\}$.

(2) $\# W(\operatorname{supp} \eta, \Theta) \times \# W_{\operatorname{supp} \eta}=\#\left\{w \in W(M) \mid \operatorname{supp} \eta \cap w\left(\Sigma_{M}^{+}\right)=\emptyset\right\}$.

Proof. (1) Put $\mathcal{W}=\left\{w \in W(M) \mid w\left(\Sigma^{+}\right) \cap \Sigma_{\eta}^{+}=\emptyset\right\}$. Let $w_{\eta, 0}$ be the longest Weyl element of $W_{M_{\eta}}$. We will prove that the map $\mathcal{W} \rightarrow W(\operatorname{supp} \eta, \Theta)$ defined by $w \mapsto\left(w_{\eta, 0} w\right)^{-1}$ is well-defined and bijective.

To prove that the map is well-defined, let $w \in \mathcal{W}$. The equality $w\left(\Sigma^{+}\right) \cap \Sigma_{\eta}^{+}=\emptyset$ implies $\left(w_{\eta, 0} w\right)^{-1}\left(\Sigma_{\eta}^{+}\right)=w^{-1}\left(-\Sigma_{\eta}^{+}\right) \subset \Sigma^{+}$. Hence $\left(w_{\eta, 0} w\right)^{-1} \in W(\operatorname{supp} \eta)$. Moreover, $w\left(\Sigma_{M}^{+}\right) \subset \Sigma^{+}$and $w\left(\Sigma^{+}\right) \cap \Sigma_{\eta}^{+}=\emptyset$ imply that $w\left(\Sigma_{M}^{+}\right) \subset \Sigma^{+} \cap\left(\Sigma \backslash \Sigma_{\eta}^{+}\right)=$ $\Sigma^{+} \backslash \Sigma_{\eta}^{+}$. Hence $\left(w_{\eta, 0} w\right)\left(\Sigma_{M}^{+}\right) \subset \Sigma^{+} \backslash \Sigma_{\eta}^{+} \subset \Sigma^{+}$. We have $\left(w_{\eta, 0} w\right)^{-1} \in W(\Theta)^{-1}$. Finally $w\left(\Sigma_{M}^{+}\right) \subset \Sigma^{+} \backslash \Sigma_{\eta}^{+}$implies $w\left(\Sigma_{M}\right)=w\left(\Sigma_{M}^{+}\right) \cup\left(-w\left(\Sigma_{M}^{+}\right)\right) \subset \Sigma \backslash \Sigma_{\eta}$. Hence $\left(w_{\eta, 0} w\right)^{-1} \Sigma_{\eta} \cap \Sigma_{M}=w^{-1} \Sigma_{\eta} \cap \Sigma_{M}=\emptyset$.

Assume that $\left(w_{\eta, 0} w\right)^{-1} \in W(\operatorname{supp} \eta, \Theta)$. From $\left(w_{\eta, 0} w\right)^{-1}\left(\Sigma_{\eta}^{+}\right) \subset \Sigma^{+}$, we have $w^{-1}\left(\Sigma_{\eta}^{-}\right) \subset \Sigma^{+}$. Hence $\Sigma_{\eta}^{+}=-\Sigma_{\eta}^{-} \subset-w\left(\Sigma^{+}\right)=w\left(\Sigma^{-}\right)$. Thus $w\left(\Sigma^{+}\right) \cap \Sigma_{\eta}^{+}$ $=\emptyset$. Since $\left(w_{\eta, 0} w\right)^{-1} \Sigma_{\eta} \cap \Sigma_{M}=\emptyset$ we have $w\left(\Sigma_{M}\right) \cap \Sigma_{\eta}=\emptyset$. As $\left(w_{\eta, 0} w\right)\left(\Sigma_{M}^{+}\right)$ $\subset \Sigma^{+}$and $w\left(\Sigma^{+}\right) \cap \Sigma_{\eta}^{+}=\emptyset$, it follows that $w\left(\Sigma_{M}^{+}\right) \subset w_{\eta, 0}^{-1}\left(\Sigma^{+}\right) \cap\left(\Sigma \backslash \Sigma_{\eta}^{-}\right)=$ $\left(\left(\Sigma^{+} \backslash \Sigma_{\eta}^{+}\right) \cup \Sigma_{\eta}^{-}\right) \cap\left(\Sigma \backslash \Sigma_{\eta}^{-}\right)=\left(\Sigma^{+} \backslash \Sigma_{\eta}^{+}\right)$. Consequently, $w \in W(M)$.

(2) Put $\mathcal{W}=\left\{w \in W(M) \mid \operatorname{supp} \eta \cap w\left(\Sigma_{M}^{+}\right)=\emptyset\right\}$. Define a map $W(\operatorname{supp} \eta, \Theta) \times W_{\operatorname{supp} \eta} \rightarrow \mathcal{W}$ by $\left(w_{1}, w_{2}\right) \mapsto w_{2} w_{1}^{-1}$. This map is injective since $W(\operatorname{supp} \eta, \Theta) \subset W(\operatorname{supp} \eta)$. We prove that it is well-defined and surjective. Since $w_{1} \in W(\operatorname{supp} \eta, \Theta) \subset W(M)^{-1}, w_{1}^{-1}\left(\Sigma_{M}^{+}\right)=w_{1}^{-1}\left(\Sigma_{M}^{+}\right) \cap \Sigma^{+}$. As $w_{1}\left(\Sigma_{\eta}\right) \cap \Sigma_{M}=\emptyset$, we have $w_{1}^{-1}\left(\Sigma_{M}^{+}\right) \cap \Sigma^{+} \subset\left(\Sigma \backslash \Sigma_{\eta}\right) \cap \Sigma^{+}=\Sigma^{+} \backslash \Sigma_{\eta}^{+}$. Therefore $w_{2} w_{1}^{-1}\left(\Sigma_{M}^{+}\right) \subset \Sigma^{+} \backslash \Sigma_{\eta}^{+}$, so the map is well-defined. Next let $w \in \mathcal{W}$. Let $w_{1} \in W(\operatorname{supp} \eta)^{-1}$ and $w_{2} \in W_{\operatorname{supp} \eta}$ be such that $w=w_{2} w_{1}^{-1}$. Then $w_{1}^{-1}\left(\Sigma_{M}^{+}\right)=$ $w_{2}^{-1} w\left(\Sigma_{M}^{+}\right) \subset w_{2}^{-1}\left(\Sigma^{+} \backslash \Sigma_{\eta}^{+}\right)=\Sigma^{+} \backslash \Sigma_{\eta}^{+} \subset \Sigma^{+}$. Hence $w_{1} \in W(M)^{-1}$. Moreover, $w_{1}^{-1}\left(\Sigma_{M}^{+}\right) \subset \Sigma^{+} \backslash \Sigma_{\eta}^{+}$implies $w_{1}^{-1}\left(\Sigma_{M}\right) \subset \Sigma \backslash \Sigma_{\eta}$. Hence $\Sigma_{\eta} \cap w_{1}^{-1}\left(\Sigma_{M}\right)=\emptyset$. Therefore, $w_{1} \Sigma_{\eta} \cap \Sigma_{M}=\emptyset$. This implies $w_{1} \in W(\operatorname{supp} \eta, \Theta)$. 
Lemma 8.19. Assume that $\sigma$ is irreducible and finite-dimensional. Let $\widetilde{\mu}$ be the highest weight of $\sigma$ and $V$ the irreducible finite-dimensional representation of $M_{0} A_{0}$ with highest weight $\lambda+\widetilde{\mu}$. Then $\mathrm{Wh}_{0}\left(\sigma^{*}\right) \simeq V^{*}$ as $M_{0} A_{0}$-modules. In particular, $\operatorname{dim} \mathrm{Wh}_{0}\left(\sigma^{\prime}\right)=\operatorname{dim} V$.

Proof. Let $\widetilde{w}_{M, 0}$ be the longest element of $\widetilde{W_{M}}$. Then both sides have highest weight $-\widetilde{w}_{M, 0}(\widetilde{\mu}+\lambda)$ and the spaces of highest weight vectors are 1-dimensional.

As a corollary to Theorems 8.8 and 8.16 , we have the following theorem announced by T. Oshima. Define $\widetilde{\rho_{M}} \in \mathfrak{h}^{*}$ by $\widetilde{\rho_{M}}=(1 / 2) \sum_{\alpha \in \Delta_{M}^{+}} \alpha$.

Theorem 8.20. Assume that $\sigma$ is the irreducible finite-dimensional representation of $M$ with highest weight $\widetilde{\nu}$. Let $\operatorname{dim}_{M_{0}}(\lambda+\widetilde{\nu})$ be the dimension of the finitedimensional irreducible representation of $M_{0} A_{0}$ with highest weight $\lambda+\widetilde{\nu}$.

(1) Assume that for all $w \in W$ such that $w\left(\Sigma^{+} \backslash \Sigma_{M}^{+}\right) \cap \operatorname{supp} \eta=\emptyset$ the following two conditions hold:

(a) $\left\langle\check{\alpha}, \lambda+w_{0} \widetilde{\nu}\right\rangle \notin \mathbb{Z}_{\leq 0}$ for all $\alpha \in \Sigma^{+} \backslash w^{-1}\left(\Sigma_{M}^{+} \cup \Sigma_{\eta}^{+}\right)$.

(b) $\lambda-\left.\left.\widetilde{w}\left(\lambda+\widetilde{\nu}+\widetilde{\rho_{M}}\right)\right|_{\mathfrak{a}} \notin \mathbb{Z}_{\leq 0}\left(\left(\Sigma^{+} \backslash \Sigma_{M}^{+}\right) \cap w^{-1} \Sigma^{+}\right)\right|_{\mathfrak{a}} \backslash\{0\}$ for all $\widetilde{w} \in \widetilde{W}$.

Then

$$
\operatorname{dim} \mathrm{Wh}_{\eta}\left(I(\sigma, \lambda)^{\prime}\right)=\# W(\operatorname{supp} \eta, \Theta) \times\left(\operatorname{dim}_{M_{0}}(\lambda+\widetilde{\nu})\right) .
$$

(2) Assume that $(\lambda+\widetilde{\nu})-\widetilde{w}(\lambda+\widetilde{\nu}) \notin \Delta$ for all $\widetilde{w} \in \widetilde{W} \backslash \widetilde{W_{M}}$. Then

$$
\operatorname{dim} \mathrm{Wh}_{\eta}\left(\left(I(\sigma, \lambda)_{K \text {-finite }}\right)^{*}\right)=\# W(\operatorname{supp} \eta, \Theta) \times \# W_{\text {supp } \eta} \times \operatorname{dim}_{M_{0}}(\lambda+\widetilde{\nu}) .
$$

Proof. Recall that $\mathrm{Wh}_{w^{-1} \eta}\left(\sigma^{*}\right) \neq 0$ if and only if $w^{-1} \eta=0$ on $\mathfrak{m} \cap \mathfrak{n}_{0}$. This is equivalent to $\operatorname{supp} \eta \cap w\left(\Sigma_{M}^{+}\right)=\emptyset$.

(1) By Theorem 8.8, we have

$$
\mathrm{Wh}_{\eta}\left(I(\sigma, \lambda)^{\prime}\right)=\sum_{w \in W(M), w\left(\Sigma^{+} \backslash \Sigma_{M}^{+}\right) \cap \operatorname{supp} \eta=\emptyset} \operatorname{dim}_{\mathrm{Wh}_{w^{-1} \eta}}\left(\left(\sigma \otimes e^{\lambda+\rho}\right)^{\prime}\right) .
$$

Since $\sigma$ is finite-dimensional, $\left(\sigma \otimes e^{\lambda+\rho}\right)^{\prime}=\left(\sigma \otimes e^{\lambda+\rho}\right)^{*}$. Then by the above remark, $\mathrm{Wh}_{w^{-1} \eta}\left(\left(\sigma \otimes e^{\lambda+\rho}\right)^{*}\right) \neq 0$ if and only if supp $\eta \cap w_{i}\left(\Sigma_{M}^{+}\right)=\emptyset$. Moreover, if supp $\eta \cap$ $w_{i}\left(\Sigma_{M}^{+}\right)=\emptyset$, then $\operatorname{dim} \mathrm{Wh}_{\eta}\left(\left(\sigma \otimes e^{\lambda+\rho}\right)^{*}\right)=\operatorname{dim}_{\mathrm{Wh}_{0}}\left(\left(\sigma \otimes e^{\lambda+\rho}\right)^{*}\right)=\operatorname{dim}_{M_{0}}(\lambda+\widetilde{\nu})$ by Lemma 8.19. Hence we get

$$
\begin{aligned}
\operatorname{dim} \mathrm{Wh}_{\eta}\left(I(\sigma, \lambda)^{\prime}\right) \times \operatorname{dim}_{M_{0}}(\lambda+\widetilde{\nu}) \\
\quad=\#\left\{w \in W(M) \mid w\left(\Sigma \backslash \Sigma_{M}^{+}\right) \cap \operatorname{supp} \eta=\emptyset, w\left(\Sigma_{M}^{+}\right) \cap \operatorname{supp} \eta=\emptyset\right\} \\
\quad=\#\left\{w \in W(M) \mid w\left(\Sigma^{+}\right) \cap \operatorname{supp} \eta=\emptyset\right\} \times \operatorname{dim}_{M_{0}}(\lambda+\widetilde{\nu}) .
\end{aligned}
$$


By the definition of $\Sigma_{\eta}^{+}$, we have $w\left(\Sigma^{+}\right) \cap \operatorname{supp} \eta=\emptyset$ if and only if $w\left(\Sigma^{+}\right) \cap \Sigma_{\eta}^{+}=\emptyset$. Hence we get (1) by Lemma 8.18(1).

(2) By the above argument, we have

$$
\operatorname{dim} \mathrm{Wh}_{\eta}\left(I(\sigma, \lambda)^{\prime}\right)=\#\left\{w \in W(M) \mid w\left(\Sigma_{M}^{+}\right) \cap \operatorname{supp} \eta=\emptyset\right\} \times \operatorname{dim}_{M_{0}}(\lambda+\widetilde{\nu}) .
$$

Hence we get (2) by Lemma 8.18(2).

\section{Appendix A. $C^{\infty}$-functions with values in Fréchet spaces}

\section{Appendix A.1. $\mathcal{L}$-distributions and tempered $\mathcal{L}$-distributions}

Let $M$ be a $C^{\infty}$-manifold, $V$ a Fréchet space and $\mathcal{L}$ a vector bundle on $M$ with fibers $V$. We define the sheaf of $\mathcal{L}$-distributions as follows.

First we assume that $\mathcal{L}$ is trivial on $M$. Then the definition of $\mathcal{L}$-distributions is found in Kolk-Varadarajan [KV96]. (It is the continuous dual space of the space of $C^{\infty}$-functions $G \rightarrow V$ with compact support.) It is easy to see that the spaces of $\mathcal{L}$-distributions form a sheaf on $M$.

In general, let $M=\bigcup_{\lambda \in \Lambda} U_{\lambda}$ be an open covering of $M$ such that the vector bundle $\mathcal{L}$ is trivial on each $U_{\lambda}$. For an arbitrary open subset $U$ of $M$, put

$$
\mathcal{D}^{\prime}(U, \mathcal{L})=\left\{\left(x_{\lambda}\right) \in \prod_{\lambda \in \Lambda} \mathcal{D}^{\prime}\left(U \cap U_{\lambda}, \mathcal{L}\right) \mid x_{\lambda}=x_{\lambda^{\prime}} \text { on } U_{\lambda} \cap U_{\lambda^{\prime}}\right\} .
$$

This is independent of the choice of an open covering $\left\{U_{\lambda}\right\}$ and defines the sheaf of $\mathcal{L}$-distributions on $M$.

Let $X$ be a compact $C^{\infty}$-manifold such that $M$ is an open dense submanifold of $X$. Assume that there exists a vector bundle on $X$ whose restriction to $M$ is $\mathcal{L}$. (We denote this vector bundle again by $\mathcal{L}$.) In this case, we define a subspace $\mathcal{T}(M, \mathcal{L})$ of $\mathcal{D}^{\prime}(M, \mathcal{L})$ by

$$
\mathcal{T}(M, \mathcal{L})=\left\{x \in \mathcal{D}^{\prime}(M, \mathcal{L})|x=z|_{M} \text { for some } z \in \mathcal{D}^{\prime}(X, \mathcal{L})\right\} .
$$

An element of $\mathcal{T}(M, \mathcal{L})$ is called a tempered $\mathcal{L}$-distribution (cf. [Sch66]).

Remark A.1. The author does not know whether this space depends on the choice of $X$ or not. Hence, in this paper, we specify $X$ when we use the notion of a tempered $\mathcal{L}$-distribution. For example, in the main part of this paper, we consider the space of tempered distributions on $U_{i}$ (Section 2). In this case, we take $G / P$ as $X$.

For a subset $M_{0} \subset M$, put $\mathcal{D}_{M_{0}}^{\prime}(U, \mathcal{L})=\left\{x \in \mathcal{D}^{\prime}(U, \mathcal{L}) \mid \operatorname{supp} x \subset M_{0}\right\}$ and $\mathcal{T}_{M_{0}}(M, \mathcal{L})=\left\{x \in \mathcal{T}(M, \mathcal{L}) \mid \operatorname{supp} x \subset M_{0}\right\}$. Assume that $M_{0}$ is a closed 
submanifold of $M$. Then dualizing the restriction map $C_{c}^{\infty}(M, \mathcal{L}) \rightarrow C_{c}^{\infty}\left(M_{0}, \mathcal{L}\right)$, we have an injective map $\mathcal{D}^{\prime}\left(M_{0}, \mathcal{L}\right) \rightarrow \mathcal{D}_{M_{0}}^{\prime}(M, \mathcal{L})$. Via this map, we regard $\mathcal{D}^{\prime}\left(M_{0}, \mathcal{L}\right)$ as a subspace of $\mathcal{D}_{M_{0}}^{\prime}(M, \mathcal{L})$.

\section{Appendix A.2. $\mathcal{L}$-distributions with support in a subspace}

Let $M$ be the Euclidean space $\mathbb{R}^{n}=\left\{\left(x_{1}, \ldots, x_{n}\right) \in \mathbb{R}^{n}\right\}$ and $M_{0}$ the subspace $\mathbb{R}^{n-m}$ of $M$ defined by $x_{1}=\cdots=x_{m}=0$. Assume that there exists a compact $C^{\infty}$-manifold $X$ which satisfies the condition of the previous section. Let $E_{1}, \ldots, E_{m}$ be vector fields on $M$ such that:

(1) $\left.\left(E_{i} \varphi\right)\right|_{M_{0}}=\left.\left(\frac{\partial}{\partial x_{i}} \varphi\right)\right|_{M_{0}}$ for all $\varphi \in C^{\infty}(M)$.

(2) The space $\sum_{i=1}^{m} \mathbb{C} E_{i}$ is a Lie algebra.

Set $D_{i}=\partial / \partial x_{i}$. Condition (1) implies that $D_{i} T=E_{i} T$ for all $T \in \mathcal{D}^{\prime}\left(M_{0}, \mathcal{L}\right)$. We define $U_{n}\left(E_{1}, \ldots, E_{m}\right)=\sum_{k_{1}+\cdots+k_{m} \leq n} \mathbb{C} E_{1}^{k_{1}} \cdots E_{m}^{k_{m}}$ and $U\left(E_{1}, \ldots, E_{m}\right)=$ $\sum_{n} U_{n}\left(E_{1}, \ldots, E_{m}\right)$. Then the algebra $U\left(E_{1}, \ldots, E_{m}\right)$ is isomorphic to the universal enveloping algebra of $\sum_{i=1}^{m} \mathbb{C} E_{i}$. For $\alpha=\left(\alpha_{1}, \ldots, \alpha_{m}\right)$, put $E^{\alpha}=E_{1}^{\alpha_{1}} \cdots E_{m}^{\alpha_{m}}$ where $E_{i}^{0}=1$.

Lemma A.2. Let $E_{1}^{\prime}, \ldots, E_{m}^{\prime}$ be vector fields on $M$ which satisfy the same conditions as $E_{1}, \ldots, E_{m}$. Then

$$
E^{\alpha} T \in\left(E^{\prime}\right)^{\alpha} T+U_{|\alpha|-1}\left(E_{1}^{\prime}, \ldots, E_{m}^{\prime}\right) \mathcal{D}^{\prime}\left(M_{0}, \mathcal{L}\right)
$$

for $T \in \mathcal{D}^{\prime}\left(M_{0}, \mathcal{L}\right)$ and $\alpha \in \mathbb{Z}_{\geq 0}^{m}$.

Proof. First we remark that if the order of a differential operator $P$ is at most $k$, then $P\left(\mathcal{D}^{\prime}\left(M_{0}, \mathcal{L}\right)\right) \subset U_{k}\left(D_{1}, \ldots, D_{m}\right) \mathcal{D}^{\prime}\left(M_{0}, \mathcal{L}\right)$. Take $P \in U_{k-1}\left(E_{1}, \ldots, E_{m}\right)$. Then

$$
\begin{array}{r}
E_{i} P T=P E_{i} T+\left[E_{i}, P\right] T=P D_{i} T+\left[E_{i}, P\right] T=D_{i} P T+\left[E_{i}-D_{i}, P\right] T \\
\in D_{i} P T+U_{k-1}\left(D_{1}, \ldots, D_{m}\right) \mathcal{D}^{\prime}\left(M_{0}, \mathcal{L}\right)
\end{array}
$$

since the order of $\left[E_{i}-D_{i}, P\right]$ is less than or equal to $k-1$. Hence, using induction on $|\alpha|$, we have $E^{\alpha} T \in D^{\alpha} T+U_{|\alpha|-1}\left(D_{1}, \ldots, D_{m}\right) \mathcal{D}^{\prime}\left(M_{0}, \mathcal{L}\right)$.

Hence $U_{k}\left(E_{1}, \ldots, E_{m}\right) \mathcal{D}^{\prime}\left(M_{0}, \mathcal{L}\right) \subset U_{k}\left(D_{1}, \ldots, D_{m}\right) \mathcal{D}^{\prime}\left(M_{0}, \mathcal{L}\right)$. Therefore,

$$
E^{\alpha} T+U_{|\alpha|-1}\left(E_{1}, \ldots, E_{m}\right) \mathcal{D}^{\prime}\left(M_{0}, \mathcal{L}\right) \subset D^{\alpha} T+U_{|\alpha|-1}\left(D_{1}, \ldots, D_{m}\right) \mathcal{D}^{\prime}\left(M_{0}, \mathcal{L}\right) .
$$

By the same argument,

$$
E^{\alpha} T+U_{|\alpha|-1}\left(E_{1}, \ldots, E_{m}\right) \mathcal{D}^{\prime}\left(M_{0}, \mathcal{L}\right) \supset D^{\alpha} T+U_{|\alpha|-1}\left(D_{1}, \ldots, D_{m}\right) \mathcal{D}^{\prime}\left(M_{0}, \mathcal{L}\right) .
$$


Hence

$$
E^{\alpha} T+U_{|\alpha|-1}\left(E_{1}, \ldots, E_{m}\right) \mathcal{D}^{\prime}\left(M_{0}, \mathcal{L}\right)=D^{\alpha} T+U_{|\alpha|-1}\left(D_{1}, \ldots, D_{m}\right) \mathcal{D}^{\prime}\left(M_{0}, \mathcal{L}\right)
$$

The same formulas hold for $E_{1}^{\prime}, \ldots, E_{m}^{\prime}$. Consequently,

$$
\begin{aligned}
E^{\alpha} T \in D^{\alpha} T+U_{|\alpha|-1}\left(D_{1}\right. & \left., \ldots, D_{m}\right) \mathcal{D}^{\prime}\left(M_{0}, \mathcal{L}\right) \\
& =\left(E^{\prime}\right)^{\alpha} T+U_{|\alpha|-1}\left(D_{1}, \ldots, D_{m}\right) \mathcal{D}^{\prime}\left(M_{0}, \mathcal{L}\right) \\
& =\left(E^{\prime}\right)^{\alpha} T+U_{|\alpha|-1}\left(E_{1}^{\prime}, \ldots, E_{m}^{\prime}\right) \mathcal{D}^{\prime}\left(M_{0}, \mathcal{L}\right)
\end{aligned}
$$

Proposition A.3. (1) The map $\Phi: U\left(E_{1}, \ldots, E_{m}\right) \otimes \mathcal{D}^{\prime}\left(M_{0}, \mathcal{L}\right) \rightarrow \mathcal{D}_{M_{0}}^{\prime}(M, \mathcal{L})$ defined by $P \otimes T \mapsto P T$ is injective.

(2) $\mathcal{T}_{M_{0}}(M, \mathcal{L}) \subset \operatorname{Im} \Phi$. Hence we have an injective homomorphism

$$
\mathcal{T}_{M_{0}}(M, \mathcal{L}) \hookrightarrow U\left(E_{1}, \ldots, E_{m}\right) \mathcal{D}^{\prime}\left(M_{0}, \mathcal{L}\right) \simeq U\left(E_{1}, \ldots, E_{m}\right) \otimes \mathcal{D}^{\prime}\left(M_{0}, \mathcal{L}\right)
$$

Proof. (1) Let $\sum_{\alpha \in \mathbb{Z}_{\geq 0}^{m}} E^{\alpha} \otimes T_{\alpha}$ be an element of $U\left(E_{1}, \ldots, E_{m}\right) \otimes \mathcal{D}^{\prime}\left(M_{0}, \mathcal{L}\right)$. Set $T=\sum_{\alpha \in \mathbb{Z}_{\geq 0}^{m}} E^{\alpha} T_{\alpha}$ and assume that $T=0$. Put $k=\max \left\{|\alpha| \mid T_{\alpha} \neq 0\right\}$. We will prove that $k=-\infty$. Assume that $k \geq 0$. By Lemma A.2, if $|\alpha|=k$ then $E^{\alpha} T_{\alpha} \in D^{\alpha} T_{\alpha}+U_{k-1}\left(D_{1}, \ldots, D_{m}\right) \mathcal{D}^{\prime}\left(M_{0}, \mathcal{L}\right)$. There exist $T_{\alpha}^{\prime}(|\alpha|<k)$ such that $\sum_{\alpha \in \mathbb{Z}_{\geq 0}^{m}} E^{\alpha} T_{\alpha}=\sum_{\alpha \in \mathbb{Z}_{\geq 0}^{m},|\alpha|<k} D^{\alpha} T_{\alpha}^{\prime}+\sum_{\alpha \in \mathbb{Z}_{\geq 0}^{m},|\alpha|=k} D^{\alpha} T_{\alpha}$. Fix $\beta \in \mathbb{Z}_{\geq 0}^{m}$ such that $|\bar{\beta}|=k$ and $f \in C_{c}^{\infty}\left(M_{0}, \mathcal{L}\right)$. Define a function $\varphi_{\beta, f}$ on $M$ by

$$
\varphi_{\beta, f}\left(x_{1}, \ldots, x_{n}\right)=x_{1}^{\beta_{1}} \cdots x_{m}^{\beta_{m}} f\left(0, \ldots, 0, x_{m+1}, \ldots, x_{n}\right) .
$$

Then $0=\langle T, \varphi\rangle=\beta_{1} ! \cdots \beta_{m} !\left\langle T_{\beta}, f\right\rangle$. Since $f$ is arbitrary, we have $T_{\beta}=0$ for all $\beta$ such that $|\beta|=k$. This is a contradiction.

(2) For a differential operator $P$, let $\operatorname{ord}(P)$ be its order. Let $S \in \mathcal{T}_{M_{0}}(M, \mathcal{L})$. By [KV96, (2.8)], for any $p \in M_{0}$ there exist an open subset $U_{p} \ni p$ and $T_{\alpha, p} \in$ $\mathcal{D}^{\prime}\left(U_{p} \cap M_{0}, \mathcal{L}\right)$ such that $\left.S\right|_{U_{p}}=\sum_{\alpha \in \mathbb{Z}_{\geq 0}^{m}} E^{\alpha} T_{\alpha, p}$ (finite sum). Let $\widetilde{S} \in \mathcal{D}^{\prime}(M, \mathcal{L}$ ) be such that $\left.\widetilde{S}\right|_{M}=S$. Since the support of $\widetilde{S}$ is compact, there exists $r \in \mathbb{Z}_{\geq 0}$ such that if $\varphi \in C_{c}^{\infty}(X, \mathcal{L})$ satisfies $\left.P \varphi\right|_{\text {supp } \widetilde{S}}=0$ for each differential operator $P$ with $\operatorname{ord}(P) \leq r$, then $\langle\widetilde{S}, \varphi\rangle=0$. (When $\mathcal{L}=\mathbb{C}$, this is [Sch66, Ch. 3, $\S 7$, Th. XXVIII]. The same proof applies.) Then $S$ has the same property. Fix $p \in M_{0}$. Set $k=\max \left\{|\alpha| \mid T_{\alpha, p} \neq 0\right\}$. Assume that $k>r$. Then for $\beta \in \mathbb{Z}_{\geq 0}^{m}$ such that $|\beta|=k,\left.P \varphi_{\beta, f}\right|_{M_{0}}=0$ for each differential operator $P$ with $\operatorname{ord}(P) \leq r$. However, by the proof of (1), we have $\left\langle S, \varphi_{\beta, f}\right\rangle \neq 0$ for some $f$. This is a contradiction. Hence $k \leq r$ for each $p \in M_{0}$. By the proof of $(1), T_{\alpha, p}=T_{\alpha, p^{\prime}}$ on $U_{p} \cap U_{p^{\prime}}$. Hence $\left\{T_{\alpha, p}\right\}_{p}$ defines a distribution $T_{\alpha}$ on $M_{0}$ and $S=\Phi\left(\sum_{|\alpha| \leq r} E^{\alpha} \otimes T_{\alpha}\right)$. 


\section{Appendix A.3. Distributions on a nilpotent Lie group}

Let $N$ be a connected, simply connected nilpotent Lie group. Put $\mathfrak{n}=\operatorname{Lie}(N)_{\mathbb{C}}$. Then the exponential map exp: $\operatorname{Lie}(N) \rightarrow N$ is a diffeomorphism. It induces the structure of a vector space on $N$. Let $\mathcal{P}(N)$ be the ring of polynomials with respect to this vector space structure (cf. Corwin and Greenleaf [CG90, §1.2]).

Let $\mathcal{L}$ be a vector bundle on $N$ whose fiber is $V$. Since $N$ is simply connected, $\mathcal{L}$ is trivial, i.e., $\mathcal{L}=N \times V$. Fix a Haar measure $d n$ on $N$. Let $V^{\prime}$ be the continuous dual space of $V$. For $F \in C^{\infty}\left(N, V^{\prime}\right)$, we define a distribution $F \delta$ by $\langle F \delta, \varphi\rangle=$ $\int_{N} F(n)(\varphi(n)) d n$ where $\varphi \in C_{c}^{\infty}(N, \mathcal{L})$. Thus we can regard $C^{\infty}\left(N, V^{\prime}\right)$ as a subspace of $\mathcal{D}^{\prime}(N, \mathcal{L})$. Let $\mathcal{P}_{k}(N)$ be the space of polynomials of degree less than or equal to $k$. Then $\mathcal{P}(N)=\sum_{k} \mathcal{P}_{k}(N)$.

Let $\eta$ be a character of $N$ and denote its differential $\mathfrak{n} \rightarrow \mathbb{C}$ again by $\eta$. Then $\eta$ can be extended to a $\mathbb{C}$-algebra homomorphism $U(\mathfrak{n}) \rightarrow \mathbb{C}$ where $U(\mathfrak{n})$ is the universal enveloping algebra of $\mathfrak{n}$. We denote this $\mathbb{C}$-algebra homomorphism again by $\eta$. Let $\operatorname{Ker} \eta$ be its kernel. For $X \in \operatorname{Lie}(N)$ and a $C^{\infty}$-function $\psi$ on $N$, put $(X \psi)(n)=\left.\frac{d}{d t} \psi(\exp (-t X) n)\right|_{t=0}$.

The algebraic tensor product $C_{c}^{\infty}(N) \otimes V$ is canonically identified with a linear subspace of $C_{c}^{\infty}(N, \mathcal{L})$ via $\varphi \otimes v \mapsto(x \mapsto \varphi(x) v)$. This subspace is dense [KV96, (2.1)].

Proposition A.4. For all $k \in \mathbb{Z}_{>0}$, there exists a positive integer $l$ such that if $T \in \mathcal{D}^{\prime}(N, \mathcal{L})$ satisfies $(\operatorname{Ker} \eta)^{k} T=0$ then $T \in\left(\mathcal{P}_{l}(N) \eta^{-1} \otimes V^{\prime}\right) \delta$. Conversely, for all $l \in \mathbb{Z}_{>0}$ there exists $k>0$ such that $(\operatorname{Ker} \eta)^{k}\left(\mathcal{P}_{l}(N) \eta^{-1} \otimes V^{\prime}\right) \delta=0$.

As a corollary, we get the following.

Corollary A.5. Let $T \in \mathcal{D}^{\prime}(N, \mathcal{L})$. Assume that there exists a positive integer $k$ such that $(\operatorname{Ker} \eta)^{k} T \in\left(\mathcal{P}(N) \eta^{-1} \otimes V^{\prime}\right) \delta$. Then $T \in\left(\mathcal{P}(N) \eta^{-1} \otimes V^{\prime}\right) \delta$.

Proof. By the second part of Proposition A.4, there exists $k^{\prime}>0$ such that $(\text { Ker } \eta)^{k^{\prime}} T=0$. Hence $T \in\left(\mathcal{P}(N) \eta^{-1} \otimes V^{\prime}\right) \delta$ by the first part.

Proof of Proposition A.4. For $T \in \mathcal{D}^{\prime}(N, \mathcal{L})$, it is easy to see that $\mathfrak{n}^{k}(T \eta)=0$ if and only if $(\operatorname{Ker} \eta)^{k} T=0$. Therefore, we may assume that $\eta$ is trivial.

First assume that $V=\mathbb{C}$. We argue by induction on $\operatorname{dim} N$. Take an element $Z$ of the center of $\operatorname{Lie}(N)$ and a subspace $\mathfrak{n}_{0, \mathbb{R}}$ such that $\operatorname{Lie}(N)=\mathbb{R} Z \oplus \mathfrak{n}_{0, \mathbb{R}}$. Put $\mathfrak{n}_{0}=\mathfrak{n}_{0, \mathbb{R}} \otimes_{\mathbb{R}} \mathbb{C}, \mathfrak{n}^{\prime}=\mathfrak{n} / \mathbb{C} Z$ and $N^{\prime}=N / \exp (\mathbb{R} Z)$. Then the projection $\mathfrak{n} \rightarrow \mathfrak{n}^{\prime}$ gives an isomorphism $\Phi: \mathfrak{n}_{0} \rightarrow \mathfrak{n}^{\prime}$ of vector spaces. Set $\Psi=\Phi^{-1}$. We have an isomorphism $\tau: \mathbb{R} \times N^{\prime} \simeq \mathbb{R} \times \operatorname{Lie}\left(N^{\prime}\right) \simeq \mathbb{R} \times \mathfrak{n}_{0, \mathbb{R}} \simeq \mathbb{R} Z \oplus \mathfrak{n}_{0, \mathbb{R}}=\operatorname{Lie}(N) \simeq N$. An element of $\mathfrak{n}$ gives a vector field on $N$. We consider the corresponding vector 
field on $\mathbb{R} \times N^{\prime}$. Define a differential operator $D_{0}$ on $\mathbb{R} \times N^{\prime}$ by $\left(D_{0} f\right)\left(z, n^{\prime}\right)=$ $(\partial f / \partial z)\left(z, n^{\prime}\right)$.

The action of $Z$ is given by $-D_{0}$. Let $D_{Y}^{\prime}$ be the differential operator on $N^{\prime}$ given by $Y \in \mathfrak{n}^{\prime}$. For $Y_{0}, Y \in \mathfrak{n}_{0, \mathbb{R}}$ and $z, t \in \mathbb{R}$, by the Campbell-Hausdorff formula, there exists a polynomial $P_{t}\left(Y_{0}, Y^{\prime}\right)$ on $\mathbb{R} \times\left(\mathfrak{n}_{0, \mathbb{R}}\right)^{2}$ such that

$$
\exp \left(-t Y_{0}\right) \exp (z Z+Y)=\exp \left(\left(z+P_{t}\left(Y_{0}, Y\right)\right) Z+\Psi\left(Y^{\prime}\left(\Phi\left(-t Y_{0}\right), \Phi(Y)\right)\right)\right.
$$

where $Y^{\prime}: \operatorname{Lie}\left(N^{\prime}\right) \times \operatorname{Lie}\left(N^{\prime}\right) \rightarrow \operatorname{Lie}\left(N^{\prime}\right)$ is given by

$$
\exp \left(Y_{0}\right) \exp (Y)=\exp \left(Y^{\prime}\left(Y_{0}, Y\right)\right)
$$

Hence the action of $Y_{0}$ is given by $P\left(Y_{0}, n^{\prime}\right) D_{0}+D_{\Phi\left(Y_{0}\right)}^{\prime}$ for a polynomial $P$.

Now we prove the first part of the proposition when $V=\mathbb{C}$. Since $\left(-D_{0}\right)^{l} T$ $=0$ for some $l$, we have $T\left(z, n^{\prime}\right)=\sum_{p=0}^{l} z^{p} T_{p}\left(n^{\prime}\right)$ for some distributions $T_{p}$ on $N^{\prime}$. By inductive hypothesis and Remark 2.2, it is sufficient to prove that for all $Y \in \mathfrak{n}^{\prime}$ there exists a positive integer $k^{\prime}$ such that $Y^{k^{\prime}} T_{p}$ is a polynomial. (See also the proof of Corollary A.5.) We prove this by induction on $p$. Set $Y_{0}=\Psi(Y)$. Since the action of $Y_{0}$ is given by $P\left(Y_{0}, n^{\prime}\right) D_{0}+D_{Y}^{\prime}$, we have

$$
\begin{aligned}
& Y_{0}^{k}\left(z^{s} T_{s}\right) \in \sum_{s_{0}<s} z^{s_{0}} C^{\infty}\left(N^{\prime}\right)+z^{s}\left(D_{Y}^{\prime}\right)^{k}\left(T_{s}\right) \quad(s \leq p), \\
& Y_{0}^{k}\left(z^{s} T_{s}\right) \in \sum_{s_{0}<s} z^{s_{0}} \mathcal{P}\left(N^{\prime}\right)+z^{s}\left(D_{Y}^{\prime}\right)^{k}\left(T_{s}\right) \quad(s>p)
\end{aligned}
$$

since $T_{s}$ is a polynomial if $s>p$ (inductive hypothesis). Take $k$ such that $Y_{0}^{k} T=0$. Then

$$
0=Y_{0}^{k} T \in \sum_{s<p} z^{s} C^{\infty}\left(N^{\prime}\right)+\sum_{s=0}^{l} z^{s} \mathcal{P}\left(N^{\prime}\right)+z^{p}\left(D_{Y}^{\prime}\right)^{k}\left(T_{s}\right) .
$$

Therefore, $\left(D_{Y}^{\prime}\right)^{k}\left(T_{s}\right) \in \mathcal{P}\left(N^{\prime}\right)$. The second part follows from [Goo76, 2.3, Corollary 2].

Fix a basis $\left\{e_{1}, \ldots, e_{n}\right\}$ of $\operatorname{Lie}(N)$. The map $\mathbb{R}^{n} \rightarrow N$ defined by $\left(x_{1}, \ldots, x_{n}\right)$ $\mapsto \exp \left(x_{1} e_{1}+\cdots+x_{n} e_{n}\right)$ is an isomorphism. Using this map, we introduce a coordinate $\left(x_{1}, \ldots, x_{n}\right)$ of $N$.

Fix $v \in V$ and consider an ordinary distribution $T_{v}: \varphi \mapsto\langle T, \varphi \otimes v\rangle$ for $\varphi \in C_{c}^{\infty}(N)$. If $\mathfrak{n}^{k} T=0$, then $\mathfrak{n}^{k} T_{v}=0$. Hence for some $l$, we have $T_{v}=\sum_{\alpha_{1}+\cdots+\alpha_{n} \leq l}\left(x_{1}^{\alpha_{1}} \cdots x_{n}^{\alpha_{n}} \otimes c_{v, \alpha_{1}, \ldots, \alpha_{n}}\right) \delta$, where $c_{v, \alpha_{1}, \ldots, \alpha_{n}} \in \mathbb{C}$. The map $v \mapsto c_{v, \alpha_{1}, \ldots, \alpha_{n}}$ is continuous linear. Hence it defines an element of $V^{\prime}$; denote it by $v_{\alpha_{1}, \ldots, \alpha_{n}}^{\prime}$. Then for $\varphi \in C_{c}^{\infty}(N)$ and $v \in V$ we have $\langle T, \varphi \otimes v\rangle=$ $\left\langle\left(\sum_{\alpha_{1}+\cdots+\alpha_{n} \leq l} x_{1}^{\alpha_{1}} \cdots x_{n}^{\alpha_{n}} \otimes v_{\alpha_{1}, \ldots, \alpha_{n}}^{\prime}\right) \delta, \varphi \otimes v\right\rangle$. Since $C_{c}^{\infty}(N) \otimes V$ is dense in $C_{c}^{\infty}(N, \mathcal{L})$, we have $T=\left(\sum_{\alpha_{1}+\cdots+\alpha_{n} \leq l} x_{1}^{\alpha_{1}} \cdots x_{n}^{\alpha_{n}} \otimes v_{\alpha_{1}, \ldots, \alpha_{n}}^{\prime}\right) \delta$. 
We now prove the second part of the proposition. For $X \in \mathfrak{n}, f \in \mathcal{P}_{l}(N)$ and $v^{\prime} \in V^{\prime}$, we have $X\left(\left(f \otimes v^{\prime}\right) \delta\right)=\left((X f) \otimes v^{\prime}\right) \delta$. Hence we may assume that $V=\mathbb{C}$.

\section{Acknowledgments}

The author is grateful to his advisor Hisayosi Matumoto for his advice and support. This research was supported by the Japan Society for the Promotion of Science Research Fellowships for Young Scientists.

\section{References}

[Abe08] N. Abe, Jacquet modules of principal series generated by the trivial $K$-type, Publ. RIMS Kyoto Univ. 44 (2008), 1169-1197. Zbl 1168.22013 MR 2477908

[AL03] H. H. Andersen and N. Lauritzen, Twisted Verma modules, in Studies in memory of Issai Schur (Chevaleret/Rehovot, 2000), Progr. Math. 210, Birkhäuser Boston, Boston, MA, 2003, 1-26. Zbl 1079.17002 MR 1985191

[Ark04] S. Arkhipov, Algebraic construction of contragradient quasi-Verma modules in positive characteristic, in Representation theory of algebraic groups and quantum groups, Adv. Stud. Pure Math. 40, Math. Soc. Japan, Tokyo, 2004, 27-68. Zbl 1096.17003 MR 2074588

[Cas80] W. Casselman, Jacquet modules for real reductive groups, in Proceedings of the International Congress of Mathematicians (Helsinki, 1978), Acad. Sci. Fennica, Helsinki, 1980, 557-563. Zbl 0425.22019 MR 0562655

[Cas89] Canonical extensions of Harish-Chandra modules to representations of $G$, Canad. J. Math. 41 (1989), 385-438. Zbl 0702.22016 MR 1013462

[CHM00] W. Casselman, H. Hecht, and D. Miličić, Bruhat filtrations and Whittaker vectors for real groups, in The mathematical legacy of Harish-Chandra (Baltimore, MD, 1998), Proc. Sympos. Pure Math. 68, Amer. Math. Soc., Providence, RI, 2000, 151-190. Zbl 0959.22010 MR 1767896

[CG90] L. J. Corwin and F. P. Greenleaf, Representations of nilpotent Lie groups and their applications. Part I, Cambridge Stud. Adv. Math. 18, Cambridge Univ. Press, Cambridge, 1990. Zbl 0704.22007 MR 1070979

[Goo76] R. W. Goodman, Nilpotent Lie groups: structure and applications to analysis, Lecture Notes in Math. 562, Springer, Berlin, 1976. Zbl 0347.22001 MR 0442149

[Jac67] H. Jacquet, Fonctions de Whittaker associées aux groupes de Chevalley, Bull. Soc. Math. France 95 (1967), 243-309. Zbl 0155.05901 MR 0271275

[Kna01] A. W. Knapp, Representation theory of semisimple groups, Princeton Landmarks in Math., Princeton Univ. Press, Princeton, NJ, 2001. Zbl 0993.22001 MR 1880691

[KS80] A. W. Knapp and E. M. Stein, Intertwining operators for semisimple groups. II, Invent. Math. 60 (1980), 9-84. Zbl 0454.22010 MR 0582703

[KV96] J. A. C. Kolk and V. S. Varadarajan, On the transverse symbol of vectorial distributions and some applications to harmonic analysis, Indag. Math. (N.S.) 7 (1996), 67-96. Zbl 0892.22010 MR 1621372

[Kos61] B. Kostant, Lie algebra cohomology and the generalized Borel-Weil theorem, Ann. of Math. (2) 74 (1961), 329-387. Zbl 0134.03501 MR 0142696 
[Kos78] _ On Whittaker vectors and representation theory, Invent. Math. 48 (1978), 101-184. Zbl 0405.22013 MR 0507800

[Lyn79] T. E. Lynch, Generalized Whittaker vectors and representation theory, Ph.D. thesis, Massachusetts Institute of Technology, 1979.

[Mat90] H. Matumoto, Whittaker modules associated with highest weight modules, Duke Math. J. 60 (1990), 59-113. Zbl 0716.17007 MR 1084467

[Sch66] L. Schwartz, Théorie des distributions, Hermann, Paris, 1966. Z Zbl 0149.09501 MR 0209834

[Wal83] N. R. Wallach, Asymptotic expansions of generalized matrix entries of representations of real reductive groups, in: Lie group representations, I (College Park, MD, 1982/1983), Lecture Notes in Math. 1024, Springer, Berlin, 1983, 287-369. Zbl 0553.22005 MR 0727854

[Wal88] Real reductive groups. I, Pure Appl. Math. 132, Academic Press, Boston, MA, 1988. Zbl 0666.22002 MR 0929683

[Yam86] H. Yamashita, On Whittaker vectors for generalized Gel'fand-Graev representations of semisimple Lie groups, J. Math. Kyoto Univ. 26 (1986), 263-298. Z Zbl 0613.22002 MR 0849220 